\title{
Five-brane webs, Higgs branches and unitary/orthosymplectic magnetic quivers
}

\author{
Mohammad Akhond, ${ }^{a}$ Federico Carta, ${ }^{b}$ Siddharth Dwivedi, ${ }^{c}$ Hirotaka Hayashi, ${ }^{d}$ \\ Sung-Soo $\mathrm{Kim}^{e}$ and Futoshi Yagi ${ }^{f}$ \\ ${ }^{a}$ Department of Physics, Swansea University, \\ Singleton Park, Swansea, SA2 8PP, U.K. \\ ${ }^{b}$ Deutches Electronen-Synchrotron, DESY, \\ Notkestraße 85, 22607 Hamburg, Germany \\ ${ }^{c}$ Center for Theoretical Physics, College of Physical Science and Technology, \\ Sichuan University, \\ Chengdu, 610064, China \\ ${ }^{d}$ Department of Physics, School of Science, Tokai University, \\ 4-1-1 Kitakaname, Hiratsuka-shi, Kanagawa 259-1292, Japan \\ e School of Physics, University of Electronic Science and Technology of China, \\ No.4, section 2, North Jianshe Road, Chengdu, Sichuan 610054, China \\ ${ }^{f}$ School of Mathematics, Southwest Jiaotong University, \\ West zone, High-tech district, Chengdu, Sichuan 611756, China \\ E-mail: akhondmohammad@gmail.com, federico.carta@desy.de, \\ sdwivedi@scu.edu.cn, h.hayashi@tokai.ac.jp, sungsoo.kim@uestc.edu.cn, \\ futoshi_yagi@swjtu.edu.cn
}

ABSTRACT: We study the Higgs branch of 5d superconformal theories engineered from brane webs with orientifold five-planes. We propose a generalization of the rules to derive magnetic quivers from brane webs pioneered in [1], by analyzing theories that can be described with a brane web with and without O5 planes. Our proposed magnetic quivers include novel features, such as hypermultiplets transforming in the fundamental-fundamental representation of two gauge nodes, antisymmetric matter, and $\mathbb{Z}_{2}$ gauge nodes. We test our results by computing the Coulomb and Higgs branch Hilbert series of the magnetic quivers obtained from the two distinct constructions and find agreement in all cases.

Keywords: Brane Dynamics in Gauge Theories, Supersymmetric Gauge Theory, Field Theories in Higher Dimensions, Field Theories in Lower Dimensions

ARXiv EPrint: 2008.01027 


\section{Contents}

1 Introduction 1

2 Magnetic quivers from $\mathrm{O5}^{-}-\mathrm{O5}^{-} \quad 4$

2.1 The $\#_{M, N}$ theory 4

2.2 The $K_{N}$ family 6

2.3 The $\mathrm{Y}_{N}$ family 15

$\begin{array}{lll}2.3 .1 & \text { The } Y_{N}^{1,1} \text { theory } & 15\end{array}$

$\begin{array}{ll}\text { 2.3.2 The } \mathrm{Y}_{N}^{2 \times 1,2 \times 1} \text { theory } & 20\end{array}$

2.4 The $H_{N}$ family 28

3 Magnetic quivers from $\mathrm{O5}^{+}-\mathrm{O5}^{+} \quad 30$

$\begin{array}{ll}3.1 \text { The }+_{1, N} \text { theory } & 30\end{array}$

$\begin{array}{ll}3.2 & \text { The } \hat{K}_{N}^{1} \text { theory } \\ 3.32\end{array}$

3.3 The $X_{N}^{1,1}$ theory 34

4 Magnetic quivers from $\mathrm{O5}^{-}-\mathrm{O5}^{+} \quad 36$

4.1 The $+\stackrel{3,1}{N}$ theory 36

4.2 The $\tilde{K}_{N}^{1}$ theory 40

5 Magnetic quivers from $\widetilde{\mathrm{O5}}^{+} \quad 43$

$5.1 \widetilde{\mathrm{O} 5}^{+}-\widetilde{\mathrm{O} 5}^{+} \quad 43$

$5.2 \widetilde{\mathrm{O} 5}^{+}-\mathrm{O}^{+} \quad 44$

6 Conclusion 46

$\begin{array}{ll}\text { A Hilbert series computations } & 47\end{array}$

A.1 Coulomb branch Hilbert series $\quad 47$

A.2 Higgs branch Hilbert series 50

B Palindromic polynomials

C More on the number of charge 2 hypermultiplets $\quad 54$



\section{Introduction}

It has been known for some time that there are interacting UV fixed points of the renormalization group (RG) in five dimensions [2-4]. Many of these superconformal theories (SCFTs) admit a relevant deformation whose low energy dynamics is captured effectively by an $\mathcal{N}=1$ gauge theory, despite the fact that Yang-Mills (YM) interactions are powercounting non-renormalizable in five dimensions. A generic feature of such an RG flow is 
that the global symmetries of the fixed point theory are enhanced with respect to the manifest global symmetries of the gauge theory description, which has been confirmed by various observables such as superconformal indices, Nekrasov partition functions and topological vertex [5-24]. It was also argued from the presence of instanton operators [25-29], defined as defect operators, that have the charges associated with the topological current $J_{I}=\frac{1}{8 \pi^{2}} \operatorname{Tr} *(F \wedge F)$. This current can mix with the flavour symmetries in the UV to form a larger symmetry group. In the case of a quiver gauge theory, there are as many topological currents as the number of gauge nodes. Their algebra may be promoted to a non-abelian one, often without mixing with flavour symmetries.

The 5 -dimensional ( $5 \mathrm{~d}) \mathcal{N}=1$ gauge theories admit an embedding into type IIB string theory which is realized as 5 -brane webs $[30,31]$. The 5 -brane webs have been a powerful tool to study 5d SCFTs, as they not only provides an effective description of the SCFT at low energy, but also reveal rich non-perturbative aspects of the SCFTs such as global symmetry enhancement [32-34] and various dualities including S-duality as well as novel UV-dualities [11, 12, 14, 21, 23, 24, 35-39].

Of particular significance to this story is the Higgs branch of the moduli space of the $5 \mathrm{~d}$ theory. The Higgs branch is both sensitive to the symmetry enhancement and computable at all values of the YM coupling. It also undergoes other dramatic effects along the flow, such as the appearance of new flat directions at the UV fixed point.

A program to study Higgs branches of $5 \mathrm{~d}$ theories by relating them to Coulomb branches of $3 \mathrm{~d} \mathcal{N}=4$ quiver gauge theories, henceforth referred to as magnetic quivers, was initiated in [40,41], following earlier work [42, 43] observing similar connections. For related work on magnetic quivers, also see [44]. 5-brane webs also play an important role in constructing the $3 \mathrm{~d}$ quiver gauge theories associated to the Higgs branch. A set of rules were established in $[45,46]$ to derive the magnetic quivers directly from the 5-brane web. In particular, the stable intersection number from the substructure of the 5-brane web at the Higgs branch captures the multiplicity of edges connecting nodes of the $3 \mathrm{~d}$ quiver [45]. This was later extended to brane webs with O5-planes in [1].

In this paper we continue along this line of logic. We generalize the construction of magnetic quivers from O5-planes by adding new entries to the list of rules established in [1]. We examine theories that can be constructed both using ordinary brane webs, as well as brane webs with O5-planes. We verify the equivalence of the Coulomb branch of the magnetic quivers obtained from the two distinct constructions by a Hilbert series computation. We view the agreement in these computations as a non-trivial test of our conjectured rules. We organize our study according to the asymptotics of the brane configuration. It will be convenient to distinguish configurations by the asymptotic charges of the O5-plane. Within a given set of asymptotic O5-plane charges, we further divide theories according to $(p, q)$ charges of the asymptotic 5-branes. We use naming conventions for the various cases inspired by [47]. Our new rules translate to appearance of new qualitative features in the magnetic quivers. This includes exotic bifundamental matter and matter in the 2nd rank tensor representations. Our rules are obtained by examining several $5 \mathrm{~d}$ theories which can be constructed both using a brane web with an O5-plane as well as brane web without the orientifold. We achieve this by considering 5d Orthosymplectic (OSp) quivers with an S- 
dual description as $D_{3}=A_{3}$ type Dynkin quiver. Upon identifying deformation parameters of the ordinary web description with those of the orientifold web one can produce many daughter theories by deforming the two sides in an equivalent way. After the deformation the unitary webs may or may not admit a simple gauge theory description, though this is not important for our purposes. We can then derive magnetic quivers for the unitary web constructions following [45], which serve as a consistency check of our conjectured rules for the OSp magnetic quivers obtained from orientifold webs.

Although the original motivations for this work are as above, our study also hints towards implications for the magnetic quivers, viewed as $3 \mathrm{~d} \mathcal{N}=4$ gauge theories. In order to verify our results we performed Hilbert series computations for both the Coulomb and Higgs branches of these theories. In all cases we found an agreement between the two computations. Together with the fact that the $5 \mathrm{~d}$ origin of these theories is identical, one is tempted to conjecture that the two theories are dual as $3 \mathrm{~d} \mathcal{N}=4$ theories. However our analysis here is too simple to determine exactly in which sense the two theories are dual to each other.

The organization of the paper is as follows. We divide the content by asymptotic behavior of orientifold planes. In section 2 we start from examples which come from 5brane web diagrams with asymptotic $\mathrm{O} 5^{-}$-planes on both ends and obtain magnetic quivers from the configurations. In the course of obtaining the magnetic quivers we observe new rules. We will also compute the Hilbert series of Coulomb branches of these magnetic quivers and compare them with those which arise from ordinary web diagrams. Section 3 considers cases where the configurations have $05^{+}$-planes on both ends, and section 4 considers examples with an $\mathrm{O} 5^{-}$-plane on one end and an $\mathrm{O} 5^{+}$-plane on the other end. In section 5 , we consider some cases which involve an $\widetilde{\mathrm{O} 5}^{+}$-plane in the diagrams. Finally we summarise our conclusions together with a set of open problems that we find are worth further investigation in section 6. Appendix A summarizes the method for computing the Hilbert series of Coulomb branches and Higgs branches. Appendix B gives some details of the Coulomb branch Hilbert series in the main sections. In appendix $\mathrm{C}$ we give more support for the rule about the number of charge 2 hypermultiplets given in section 2 . Appendix D summarizes more examples from brane configurations with $\mathrm{O}^{-}$-planes on both ends.

Notation. To avoid the cluttering of the quiver diagrams, we will use a color coding to represent the unitary and orthosymplectic nodes as given below:

\begin{tabular}{|c|c|c|c|}
\hline Node type & $\mathrm{U}(n)$ & $\mathrm{SO}(m)$ & $\mathrm{USp}(2 k)$ \\
\hline Gauge & $\bigcirc n$ & $\bullet m$ & $\bullet 2 k$ \\
Flavor & $\square n$ & $\square m$ & $\square 2 k$ \\
\hline
\end{tabular}

In the above, the circular nodes denote the gauge group while the square nodes represent a global (rather than gauge) symmetry group. In this work, we will have three kinds of links connecting the nodes: solid line, dashed line and wavy line. These links transform 
under the representations of the nodes it connects with the following dictionary.

\begin{tabular}{|c|c|}
\hline Link type & Interpretation \\
\hline $\mathrm{O}-\mathrm{O}$ & hypermultiplet transforming in the bifundamental representation \\
\hline $0-0$ & hypermultiplet transforming in the bifundamental representation \\
\hline 0 & hypermultiplet transforming in the bifundamental representation \\
\hline 0 & half-hypermultiplet transforming in the bifundamental representation \\
\hline $0----0$ & hypermultiplet in the fundamental-fundamental representation \\
\hline œ & charge 2 hypermultiplet \\
\hline
\end{tabular}

In order to avoid confusion, we will denote 5 d (electric) quivers as $\cdots-G-G_{j}-\cdots$ and use square braces $[F]$ to denote flavor nodes.

\section{Magnetic quivers from $\mathrm{O5}^{-}-\mathrm{O5}^{-}$}

We first consider examples whose brane configurations are accompanied with two asymptotic $\mathrm{O} 5^{-}$-planes.

\subsection{The $\#_{M, N}$ theory}

The first example we consider is the brane configuration obtained by intersecting $M$ D 5 and $2 N$ NS5 branes on top of an O5-plane, which in this section we take to be asymptotically an $\mathrm{O}^{-}$-plane. We call the theory on the web the $\#_{M, N}$ theory. The brane web for this theory is depicted in figure 1. The effective theory on the Coulomb branch is a linear orthosymplectic quiver

$$
[M]-\overbrace{\mathrm{USp}(2 M-4)-\mathrm{SO}(2 M)-\mathrm{USp}(2 M-4)-\cdots-\mathrm{SO}(2 M)-\mathrm{USp}(2 M-4)}^{2 N-1}-[M]
$$

The corresponding magnetic quiver was in fact already derived in [1]. We will not repeat the steps here and simply recall that it is given by






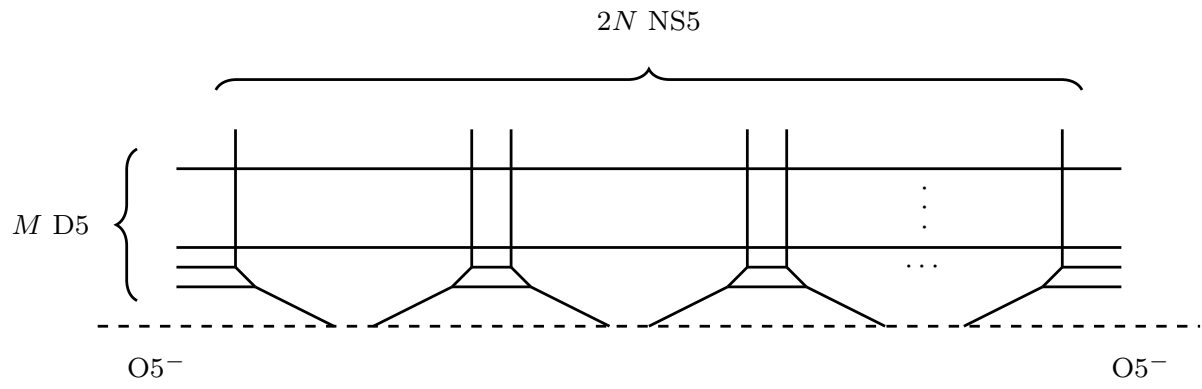

Figure 1. 5-brane web for the $\#_{M, N}$ theory.

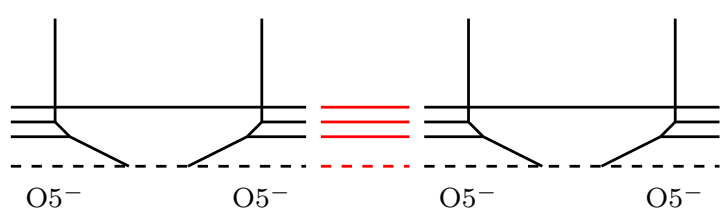

(a)



(b)

Figure 2. (a) Gluing together 2 copies of $\operatorname{USp}(2)+6 \mathbf{F}$ by gauging a common $\mathfrak{s o}(6)$ subalgebra of their global symmetry. (b) Gluing together 2 copies of $\mathrm{SU}(2)+6 \mathbf{F}$ by gauging a common $\mathfrak{s u}(4)$ subalgebra of their global symmetry.

The $5 \mathrm{~d}$ theory admits an S-dual description, as a $D$-type Dynkin quiver of special-unitary nodes $[38,48]$. In the special case when $M=3$, the S-dual theory on the Coulomb branch is

$$
\begin{gathered}
{[2 N]} \\
\stackrel{\mathrm{SU}(N)}{\mid} \mathrm{SU}(2 N)-\mathrm{SU}(N)
\end{gathered}
$$

which can also be engineered via an ordinary web diagram, without an O5-plane. One way to see this is to consider gluing together $N$ copies of $\operatorname{USp}(2)+6 \mathbf{F}$, by successive gauging $\mathfrak{s o}(6)$ subalgebra of the flavour symmetry. This should be equivalent to gluing together $N$ copies of $\mathrm{SU}(2)+6 \mathbf{F}$ by gauging $\mathfrak{s u}(4)$ subalgebra of the global symmetry. Then we perform S-duality and the diagram yields the theory in (2.3). See figure 2 for the $N=2$ example. We will make use of this construction to obtain web diagrams without O5-planes. Furthermore there are various ways to realize $\operatorname{USp}(2)$ gauge theory with six flavors depending on how we attach flavors to the diagram. Depending on situations we will use useful diagrams of $\mathrm{SU}(2)+6 \mathbf{F}$ for the $\mathfrak{s u}(4)$ gauging. The unitary web diagram for the $\#_{3, N}$ theory is shown in the figure 3 .

Given this diagram, we can immediately obtain the magnetic quiver using the rules in [45]. We are thus led to claim the equivalence of the Coulomb branch of (2.2), for $M=3$ 


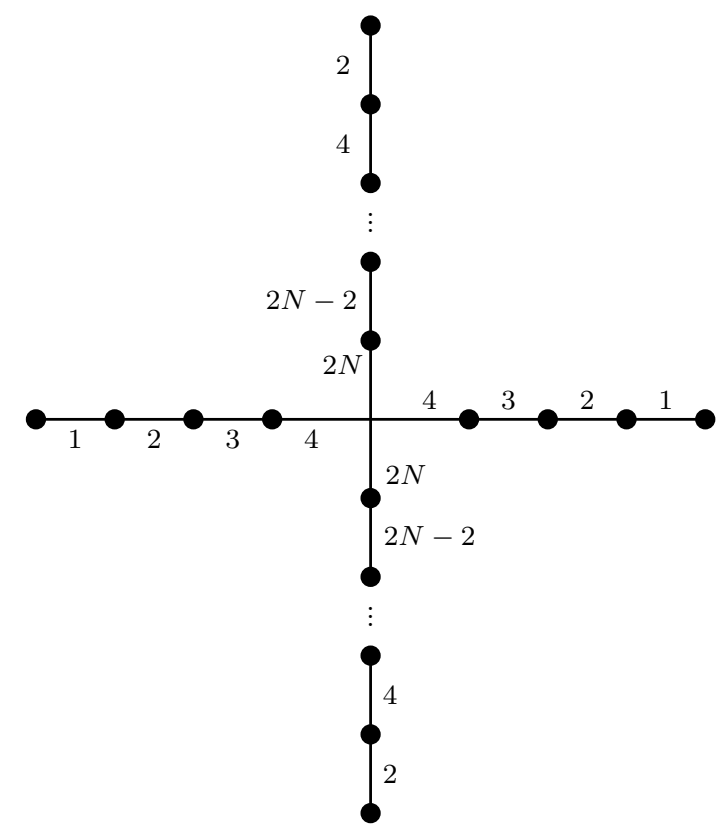

Figure 3. A unitary web realization of the $\#_{3, N}$ theory. We depict here the web at the fixed point. Black dots represent 7-branes.

with the following unitary magnetic quiver.



Both (2.4) and the $M=3$ case of (2.2) hint at an enhanced $\mathrm{SU}(2 N) \times \mathrm{SU}(4)^{2}$ flavour symmetry, which can be read off from the balanced nodes [49]. ${ }^{1}$ The Coulomb and Higgs branch dimension of both quivers are also in agreement. A further non-trivial check of our discussion is the agreement of the Hilbert series, which we have explicitly computed for low values of $N$. For $N=1$, the unitary magnetic quiver in (2.4) is well known with the Coulomb branch having $E_{7}$ as the enhanced global symmetry. We have tabulated the Coulomb branch Hilbert series for the unitary and the orthosymplectic magnetic quivers derived from the unitary and orientifold webs of $\#_{3, N}$ theory in table 1 for some small values of $N$. Note that the Hilbert series for $N=1$ is already known $([50,51])$.

\subsection{The $K_{N}$ family}

Decoupling flavors from, say, the rightmost gauge node in the $\#_{3, N}$ theory (2.1), we obtain a family of theories which we denote by $K_{N}^{p}$, where $p$ denotes the number of decoupled

\footnotetext{
${ }^{1}$ We recall the balance condition for $\mathrm{U}(r), \operatorname{USp}(2 r)$ and $\mathrm{SO}(m)$ is $n_{f}=2 r, n_{f}=2 r+1$ and $n_{f}=$ $m-1$ respectively where $n_{f}$ is the number of effective flavors. From [49], chain of $p$ balanced alternating orthosymplectic nodes give rise to an $\mathrm{SO}(p+1)$ isometry on the $\mathrm{CB}$, which is further enhanced to $\mathrm{SO}(p+2)$ if there is an $\mathrm{SO}(2)$ node at the end of the chain. A set of $p$ balanced unitary nodes which form an ADE Dynkin diagram give rise to a symmetry of the corresponding type.
} 


\begin{tabular}{|c|c|c|c|}
\hline \multirow{2}{*}{$\#_{3, N}$} & Unitary magnetic quiver & \multicolumn{2}{|c|}{ Orthosymplectic magnetic quiver } \\
\cline { 2 - 4 } & $\operatorname{HS}(t)$ & $\operatorname{HS}(t ; \vec{m} \in \mathbb{Z})$ & $\operatorname{HS}\left(t ; \vec{m} \in \mathbb{Z}+\frac{1}{2}\right)$ \\
\hline \multirow{3}{*}{$\#_{3,1}$} & $\frac{(1+t) P_{0}(t)}{(1-t)^{34}}$ & $\frac{P_{1}(t)}{(1-t)^{34}(1+t)^{17}}$ & $\frac{P_{2}(t)}{(1-t)^{34}(1+t)^{17}}$ \\
& $=1+133 t+7371 t^{2}$ & $=1+69 t+3723 t^{2}+119434 t^{3}$ & $=64 t+3648 t^{2}+119168 t^{3}$ \\
& $+238602 t^{3}+5248750 t^{4}$ & $+2625390 t^{4}+42857892 t^{5}+\cdots$ & $+2623360 t^{4}+42852096 t^{5}+\cdots$ \\
& $+85709988 t^{5}+\cdots$ & & \\
\hline \multirow{3}{*}{$\#_{3,2}$} & $1+45 t+1277 t^{2}+27399 t^{3}+$ & $1+45 t+1085 t^{2}+18951 t^{3}+$ & $192 t^{2}+8448 t^{3}+196544 t^{4}+$ \\
& $476864 t^{4}+6979468 t^{5}+$ & $280320 t^{4}+3739084 t^{5}+$ & $3240384 t^{5}+42758080 t^{6}+\cdots$ \\
\hline
\end{tabular}

Table 1. Coulomb branch Hilbert series of the unitary and orthosymplectic magnetic quivers for the $\#_{3, N}$ theory. The corresponding quivers are given in (2.4) and (2.2) respectively. For orthosymplectic quivers, we need to add the contributions of both integer and half integer fluxes. The explicit forms of the numerators $P_{0}(t), P_{1}(t), P_{2}(t)$ are provided in appendix B.

flavors. This family enjoys an IR quiver description as

$$
[3]-\overbrace{\mathrm{USp}(2)-\mathrm{SO}(6)-\mathrm{USp}(2)-\cdots-\mathrm{SO}(6)-\mathrm{USp}(2)}^{2 N-1}-[3-p]
$$

Once again, it is possible to write down an ordinary web diagram for this theory, following a gluing procedure similar to figure $2 .^{2}$ We present the orientifold and the unitary web diagrams for the family $K_{N}^{p}$ for various number of decoupled flavors which can be found in the figures mentioned below.

\begin{tabular}{|c|c|c|}
\hline Theory & Orientifold web & Unitary web \\
$K_{N}^{1}$ & Figure 4 & Figure 5 \\
$K_{N}^{2}$ & Figure 6 & Figure 7 \\
$K_{N}^{3}$ & Figure 8 & Figure 9 \\
\hline
\end{tabular}

Here, we note that these are not the only possible subdivisions. We list some examples of subdivisions and their corresponding magnetic quivers. In this paper, our focus is on extracting the rules rather than an exhaustive analysis of the Higgs branch, so we consider some of the Higgs branches rather than exhausting all the branches. ${ }^{3}$

For reading off the magnetic quivers from the unitary web diagrams in figures 5, 7, 9, we can use the rules established in [45]. For the magnetic quivers originated from the orientifold web diagrams in figures $4,6,8$, a large part of the magnetic quivers can be obtained by the rules in [1], but in fact, some part already requires an extension of the

\footnotetext{
${ }^{2}$ The cautious reader may be concerned about the non-uniqueness of this gauging procedure which is related to the Chern-Simons level of the gauging. One can remove the ambiguity by demanding that the OSp magnetic quiver agrees with the unitary quiver obtained from the unitary web after gauging. It is also possible to reproduce the same unitary web more rigorously by identifying the map between the deformation parameters in the orientifold and unitary web of the $\#_{3, N}$ theory.

${ }^{3}$ We thank the authors of [52] for informing us that they were able to find some missing cones using their computer program.
} 
rule. In [1], it has been argued that a subweb associated with a $\mathrm{U}(1)$ gauge node in a magnetic quiver which passes through the $\mathrm{O}^{-}$-plane may have charge 2 hypermultiplets coupled to the $\mathrm{U}(1)$. Such a subweb appears in the $K_{N}^{p}(p=1,2,3)$ family at the center of the junction in the orientifold diagrams, and it is depicted as the subweb in black in each maximal subdivision in figures $4,6,8$.

The subweb configuration of the maximal subdivision in figure 4 has already appeared in [1], for example, for the magnetic quiver of the rank-1 $E_{6}$ theory. In this case, the number of the charge 2 hypermultiplets attached to the U(1) node is zero. For the subweb configurations of the $K_{N}^{2}$ and $K_{N}^{3}$ theories, we find that the number of the charge 2 hypermultiplets is zero and one respectively to match the Coulomb branch Hilbert series for their magnetic quiver theories with the Coulomb branch Hilbert series for the corresponding unitary magnetic quivers. Based on these examples as well as the other examples which we will see later, we observe that the number of the charge 2 hypermultiplets may be counted by

$$
\frac{\text { SI of subweb with its own mirror image }}{2}-\mathrm{SI} \text { of subweb with } \mathrm{O}^{-} \text {, }
$$

where SI represents the stable intersection number discussed in [45]. ${ }^{4}$

Let us then illustrate how the rule (2.6) works for the subwebs of the $K_{N}^{2}$ and $K_{N}^{3}$ theories. From the maximal subdivision in figure 6 , the subweb in black at the center of the junction yields a $\mathrm{U}(1)$ gauge node. The stable intersection number of the subweb with its own mirror image is given by

$$
\text { SI of subweb with its own mirror image }=4-2=2 \text {. }
$$

On the other hand, the stable intersection number of the subweb with O5 needs some care. The subweb configuration with the orientifold is depicted in (2.8).

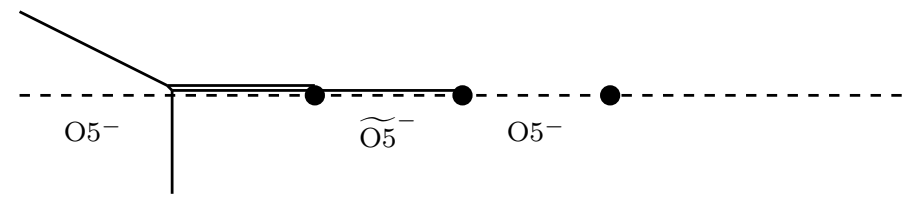

Note here that the RR charge of $\mathrm{O}^{-}$-plane is -1 and that of $\widetilde{\mathrm{O}}^{-}$-plane is $-\frac{1}{2}$ due to the half D5-brane. Then the stable intersection number of the subweb with the O5-planes becomes

$$
\text { SI of subweb with } \mathrm{O} 5^{-}=(1-1)+\left(\frac{1}{2}-\frac{1}{2}\right)+1=1
$$

The first bracket in (2.9) is the stable intersection number between the left $\mathrm{O}^{-}$-plane and the subweb in (2.8), the second bracket in (2.9) is the stable intersection number between the left $\widetilde{\mathrm{O} 5}^{-}$-plane and the subweb in (2.8), and the last 1 is the stable intersection number between the right $\mathrm{O}^{-}$-plane and the subweb in (2.8). Namely we consider the net

\footnotetext{
${ }^{4}$ In this paper, we use "SI" to denote the generalized stable intersection number, which includes the contribution from the common 7-branes, for simplicity. We call usual stable intersection without the contribution from the common 7-branes as "bare SI".
} 
contribution of the stable intersection numbers between the subweb and each piece of the orientifold. Putting together the result of (2.7) and (2.9), the (2.6) becomes

$$
\frac{2}{2}-1=0
$$

which is the right number of the charge 2 hypermultiplet coupled to the $\mathrm{U}(1)$ gauge node associated to the subweb in (2.8).

We can also do the same computation for the subweb in the maximal subdivision at the center of the junction in the $K_{N}^{3}$ theory depicted in figure 8. The stable intersection number of the subweb with its own mirror is given by

$$
\text { SI of subweb with its own mirror image }=6-3=3 \text {. }
$$

For computing the stable intersection number of the subweb with $\mathrm{O}^{-}$, we consider the configuration around the subweb depicted in (2.12).

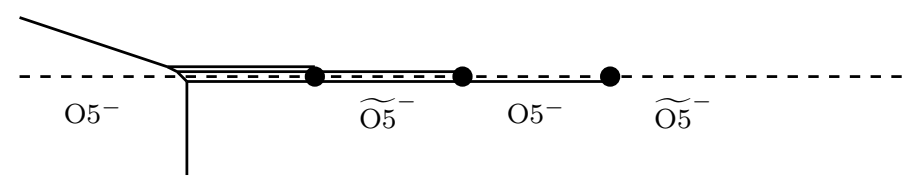

Then the stable intersection number of the subweb with the $\mathrm{O}^{-}{ }^{-}$-planes becomes

$$
\text { SI of subweb with } \mathrm{O}^{-}=(1-1)+\left(\frac{1}{2}-\frac{1}{2}\right)+(1-1)+\frac{1}{2}=\frac{1}{2} \text {. }
$$

Hence the number of the charge 2 hypermultiplets counted by (2.6) is

$$
\frac{3}{2}-\frac{1}{2}=1,
$$

which is the correct number for the charge 2 hypermultiplets coupled to the $\mathrm{U}(1)$ gauge node associated to the subweb in (2.12). The rule (2.6) also works for the subweb in the maximal subdivision at the center of the junction in the $K_{N}^{1}$ theory depicted in figure 4 .

The other parts of the magnetic quivers can be obtained from the rules established in $[1,45]$. We summarize the unitary and the orthosymplectic magnetic quiver theories derived from the unitary and the orientifold web diagrams of $K_{N}^{p}(p=1,2,3)$ family in the table 2. It is possible to compute the Coulomb branch Hilbert series for these magnetic quivers for each family in table 2 . We present some results in table 3 , and we see that the Hilbert series of the unitary and orthosymplectic quivers agree with each other. 


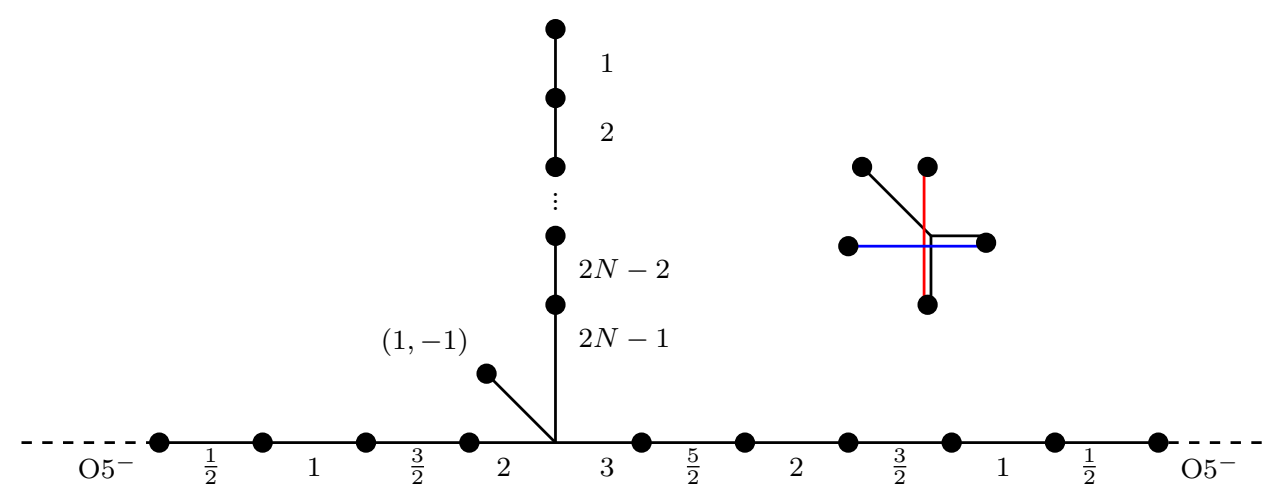

Figure 4. An orientifold web for the $\mathrm{K}_{N}^{1}$ theory and the maximal subdivision at the centre of the junction.

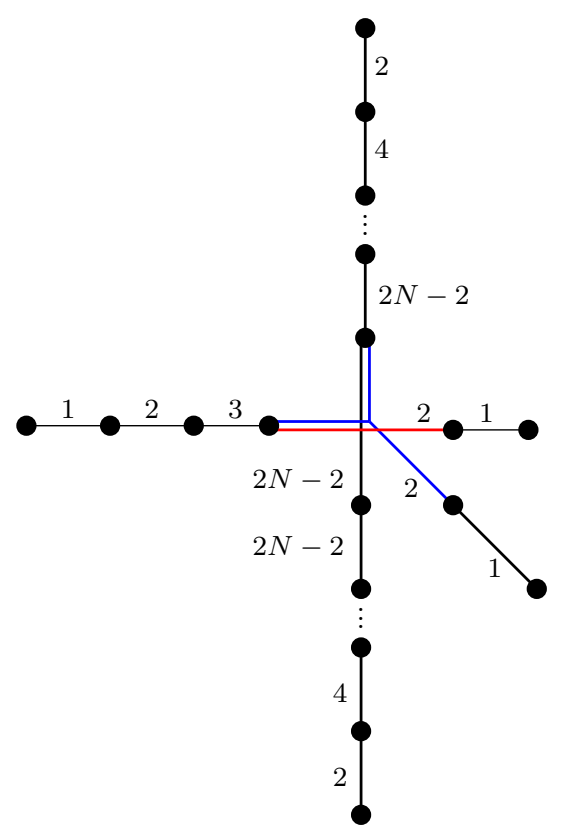

Figure 5. A unitary web for the $\mathrm{K}_{N}^{1}$ theory. The maximal subdivision leading to the magnetic quiver is indicated by use of colours.

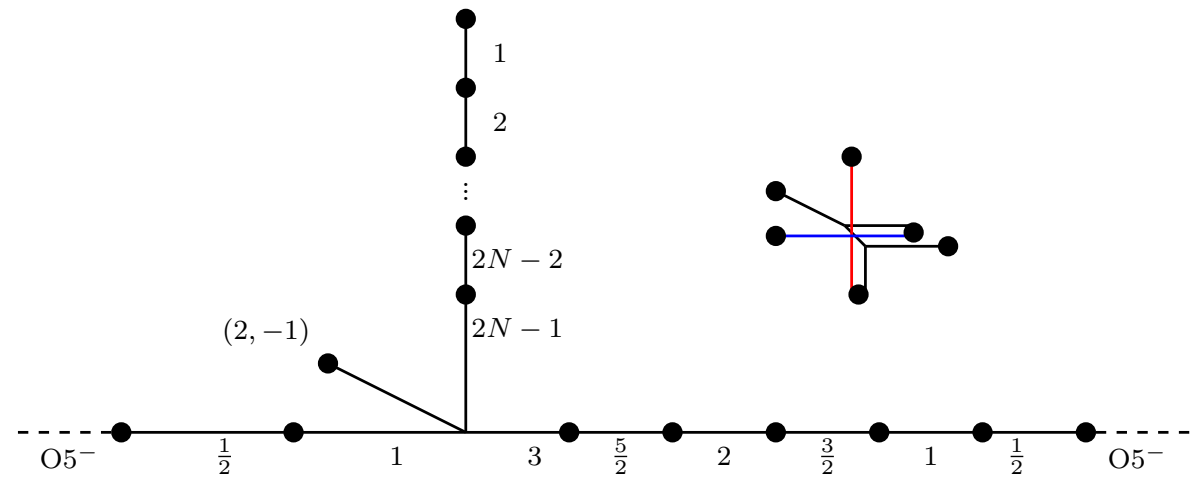

Figure 6. An orientifold web for the $\mathrm{K}_{N}^{2}$ theory and the maximal subdivision at the centre of the junction. 

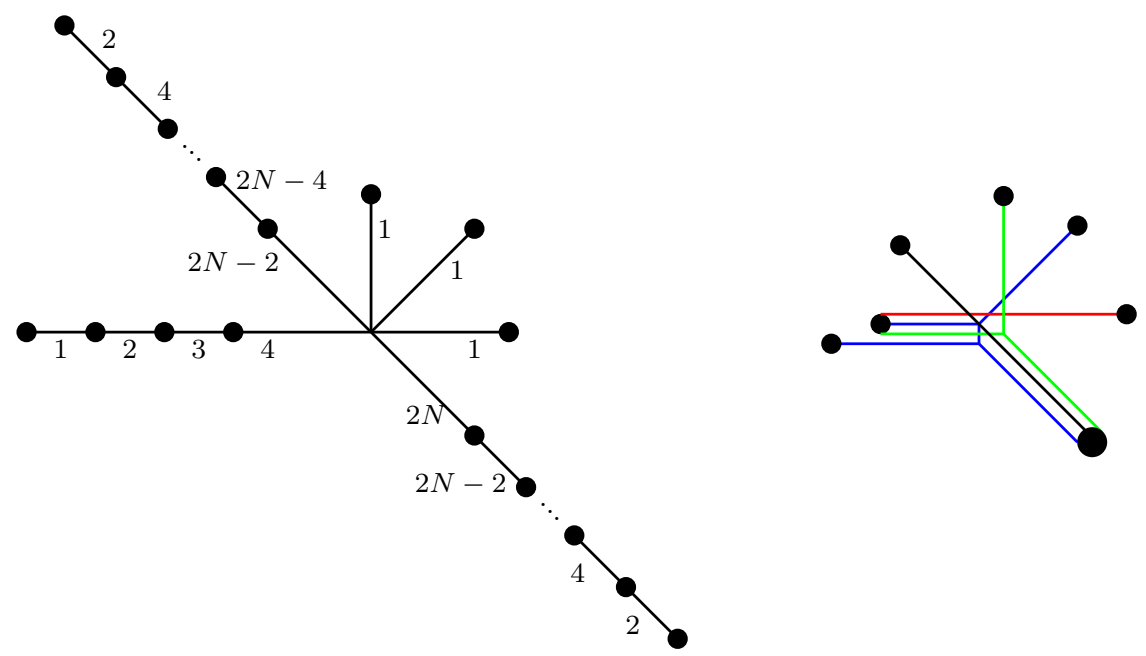

Figure 7. A unitary web description for the $\mathrm{K}_{N}^{2}$ theory, together with the maximal subdivision for the Higgs branch at infinite coupling.

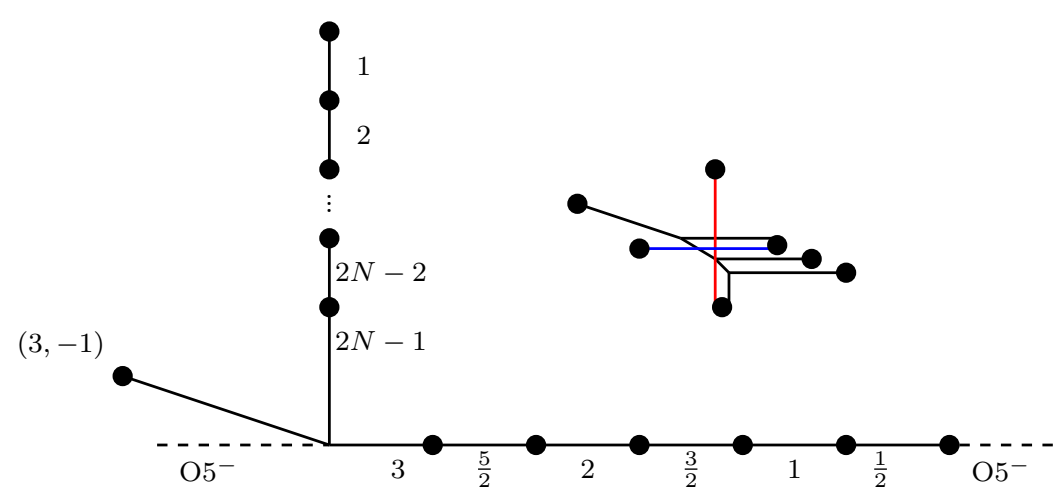

Figure 8. An orientifold web for the $\mathrm{K}_{N}^{3}$ theory and the maximal subdivision at the centre of the junction. 

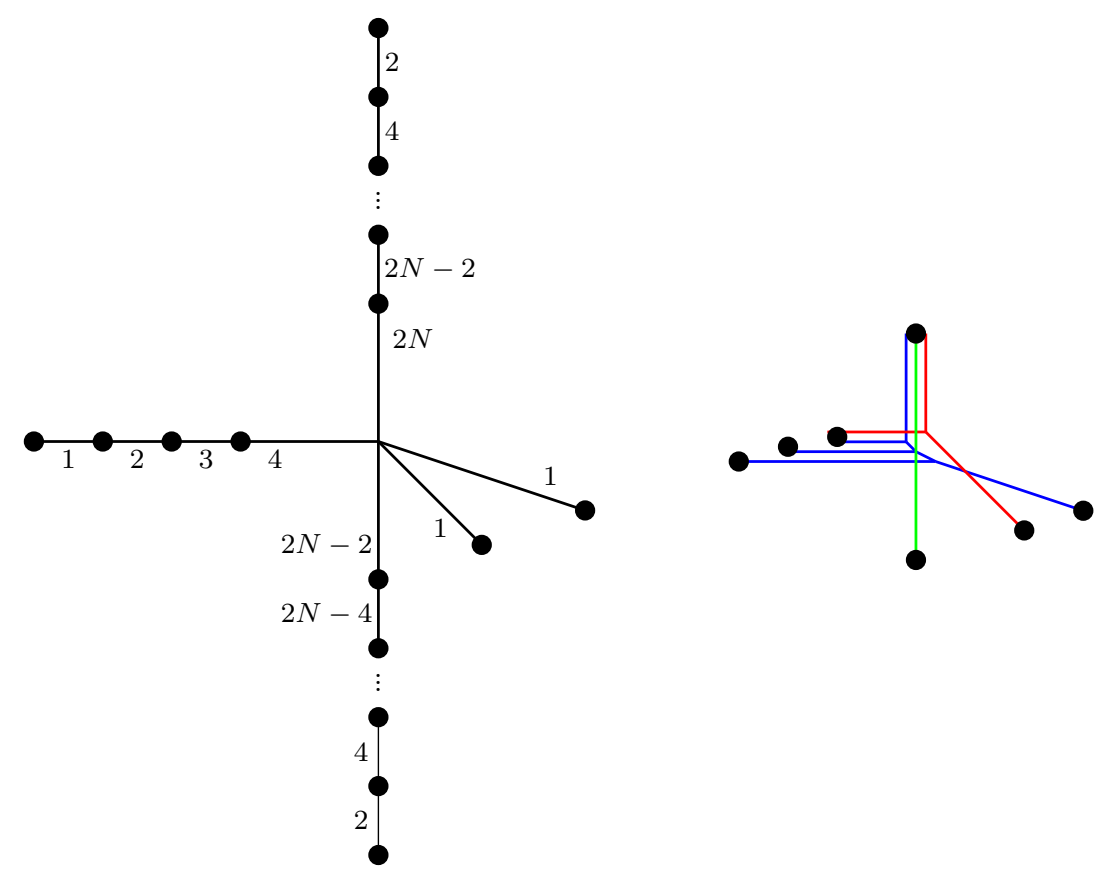

Figure 9. A unitary web description for the $\mathrm{K}_{N}^{3}$ theory, together with the maximal subdivision for the Higgs branch at infinite coupling. 


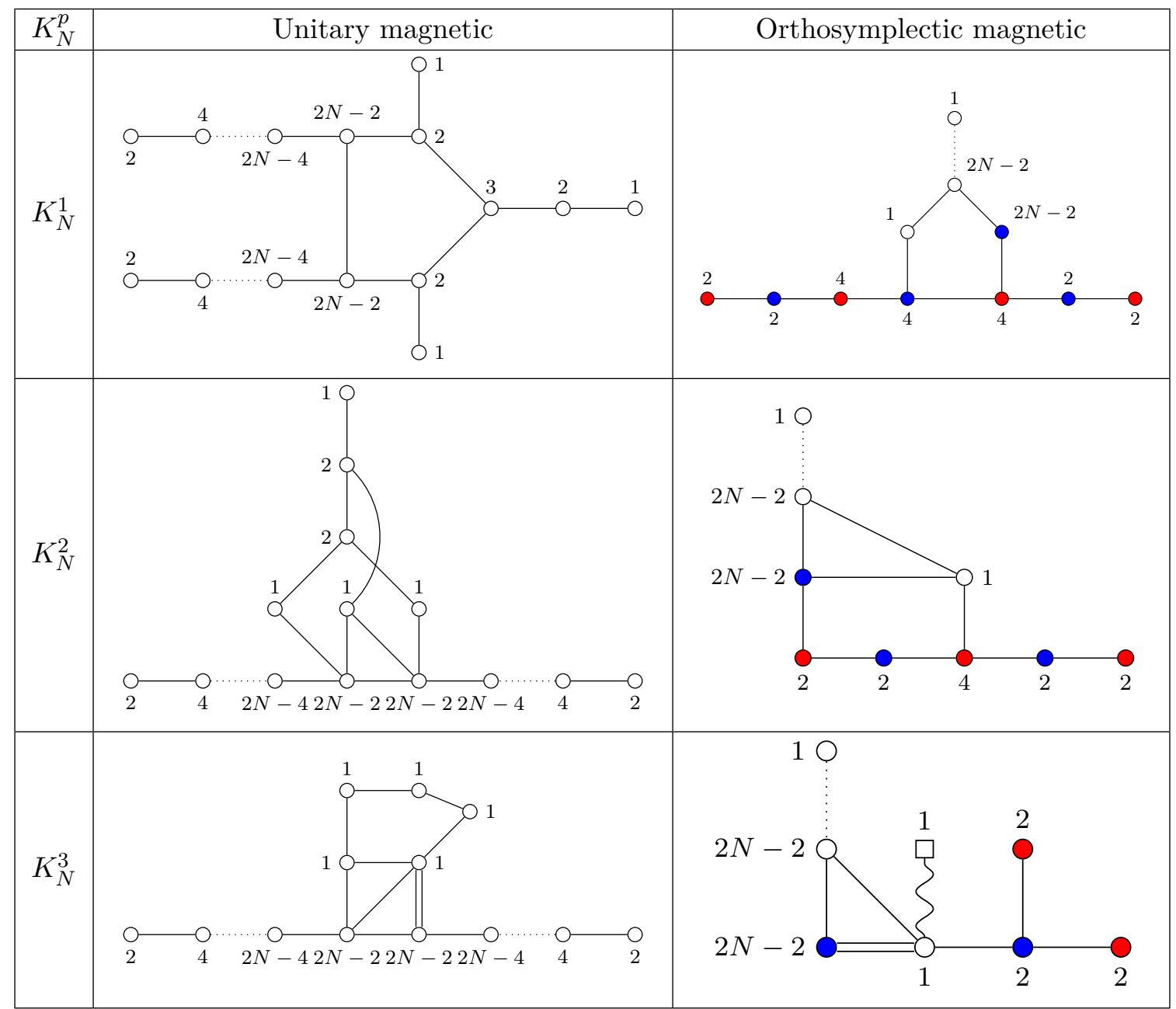

Table 2. Magnetic quivers for the $K_{N}^{p}$ family. The unitary quivers are derived from the unitary web diagrams of figures $5,7,9$. The orthosymplectic quivers on the other hand come from the orinetifold web diagrams of figures 4,6 and 8 respectively. 


\begin{tabular}{|c|c|c|c|}
\hline \multirow{2}{*}{$K_{N}^{p}$} & Unitary magnetic quiver & \multicolumn{2}{|c|}{ Orthosymplectic magnetic quiver } \\
\hline & $\mathrm{HS}(t)$ & $\mathrm{HS}(t ; \vec{m} \in \mathbb{Z})$ & $\mathrm{HS}\left(t ; \vec{m} \in \mathbb{Z}+\frac{1}{2}\right)$ \\
\hline$K_{1}^{1}$ & $\begin{array}{c}1+78 t+2430 t^{2}+43758 t^{3}+ \\
537966 t^{4}+4969107 t^{5}+\ldots\end{array}$ & $\begin{array}{c}1+46 t+1278 t^{2}+22254 t^{3}+ \\
270798 t^{4}+2491731 t^{5}+\ldots\end{array}$ & $\begin{array}{c}32 t+1152 t^{2}+21504 t^{3}+ \\
267168 t^{4}+2477376 t^{5}+\ldots\end{array}$ \\
\hline$K_{2}^{1}$ & $\begin{array}{l}1+30 t+592 t^{2}+8867 t^{3}+ \\
106965 t^{4}+1073577 t^{5}+\ldots\end{array}$ & $\begin{array}{c}1+30 t+496 t^{2}+6083 t^{3}+ \\
63477 t^{4}+586537 t^{5}+\ldots\end{array}$ & $\begin{array}{c}96 t^{2}+2784 t^{3}+43488 t^{4}+ \\
487040 t^{5}+\ldots\end{array}$ \\
\hline$K_{1}^{2}$ & $\begin{array}{l}\frac{P_{3}(t)}{(1-t)^{14}} \\
=1+45 t+770 t^{2}+7644 t^{3} \\
+52920 t^{4}+282744 t^{5}+\ldots\end{array}$ & $\begin{array}{l}\frac{P_{4}(t)}{(1-t)^{14}(1+t)^{7}} \\
=1+29 t+434 t^{2}+4060 t^{3} \\
+27384 t^{4}+144312 t^{5}+\ldots\end{array}$ & $\begin{array}{l}\frac{P_{5}(t)}{(1-t)^{14}(1+t)^{7}} \\
=16 t+336 t^{2}+3584 t^{3} \\
+25536 t^{4}+138432 t^{5}+.\end{array}$ \\
\hline$K_{2}^{2}$ & $\begin{array}{c}1+25 t+392 t^{2}+4590 t^{3}+ \\
42387 t^{4}+320549 t^{5}+\ldots\end{array}$ & $\begin{array}{c}1+25 t+344 t^{2}+3438 t^{3}+ \\
27843 t^{4}+191957 t^{5}+\ldots\end{array}$ & $\begin{array}{c}48 t^{2}+1152 t^{3}+14544 t^{4}+ \\
128592 t^{5}+\ldots\end{array}$ \\
\hline$K_{1}^{3}$ & $\begin{array}{l}\frac{1+16 t+36 t^{2}+16 t^{3}+t^{4}}{(1-t)^{8}} \\
=1+24 t+200 t^{2}+1000 t^{3} \\
+3675 t^{4}+10976 t^{5}+\ldots\end{array}$ & $\begin{array}{l}\frac{P_{6}(t)}{(1-t)^{8}(1+t)^{4}} \\
=1+16 t+120 t^{2}+560 t^{3} \\
+1995 t^{4}+5824 t^{5}+\ldots\end{array}$ & $\begin{array}{l}\frac{P_{7}(t)}{(1-t)^{8}(1+t)^{4}} \\
=8 t+80 t^{2}+440 t^{3} \\
+1680 t^{4}+5152 t^{5}+\ldots\end{array}$ \\
\hline$K_{2}^{3}$ & $\begin{array}{c}1+24 t+296 t^{2}+2510 t^{3}+ \\
16374 t^{4}+87306 t^{5}+\ldots\end{array}$ & $\begin{array}{c}1+24 t+272 t^{2}+2078 t^{3}+ \\
12294 t^{4}+60450 t^{5}+\ldots\end{array}$ & $\begin{array}{c}24 t^{2}+432 t^{3}+4080 t^{4}+ \\
26856 t^{5}+\ldots\end{array}$ \\
\hline
\end{tabular}

Table 3. Coulomb branch Hilbert series of the unitary and orthosymplectic magnetic quivers for the $K_{N}^{p}$ family listed in table 2. For orthosymplectic quivers, we need to add the contributions of both integer and half integer fluxes. The total Hilbert series then matches with that of the unitary quivers. The explicit forms of $P_{3}(t), P_{4}(t), P_{5}(t), P_{6}(t), P_{7}(t)$ are given in appendix B. 


\subsection{The $\mathrm{Y}_{N}$ family}

We then consider a different type of decoupling from the $\#_{3, N}$ theory to arrive at different examples which show some new features.

\subsubsection{The $Y_{N}^{1,1}$ theory}

In section 2.2, we decouple flavors of the $\operatorname{USp}(2)$ gauge node on one end. Here we decouple one flavor from the $\operatorname{USp}(2)$ gauge nodes on the two ends and call the theory $\mathrm{Y}_{N}^{1,1}$ theory. An IR description of the theory is

$$
[2 \overbrace{-\mathrm{USp}(2)-\mathrm{SO}(6)-\mathrm{USp}(2)-\cdots-\mathrm{SO}(6)-\mathrm{USp}(2)}^{2 N+1}-[2] .
$$

An orientifold web diagram of the $\mathrm{Y}_{N}^{1,1}$ theory is obtained by intersecting 2 D5, $2 N$ NS5, one $(1,1)$ and one $(1,-1) 5$-brane on top of an O5-plane, here taken to be asymptotically O5 ${ }^{-}$-plane (figure 10). The theory also admits a description in terms of an ordinary web which we have shown in figure 11 . The ordinary web description follows either by reading off the low energy gauge theory from an S-dual description or by following a gluing procedure similar to the one described in figure 2 .

Given the maximal subdivisions in figure 10 and figure 11, we can write down the corresponding orthosymplectic and unitary magnetic quivers, the results are collected in table 4. For the maximal subdivisions labeled as (I) and (III), the magnetic quivers are straightforward to derive. The subdivision (II) requires further clarification. Here we encounter another instance of a new feature appearing in the OSp magnetic quiver. The appearance of an exotic bi-fundamental, denoted by a dashed link in the orthosymplectic quiver in table 4 corresponding to maximal subdivision (II). To explain the origin, as well as the meaning of this link, we refer to figure 12. Here we show the subdivisions responsible for the two U(1) nodes from which this link emanates. Denoting the coordinates of the $x^{7,8,9}$ directions, which are the directions where 7-branes extend but 5-branes do not extend, of a given subweb by $\vec{m}_{i}$, its mirror image must be at coordinate $-\vec{m}_{i}$. The distance between the upper right and upper left subwebs in, say the $x^{7}$ direction, in figure 12 is therefore given by $\left|m_{1}^{(7)}-m_{2}^{(7)}\right|$. We claim that this gives rise to an ordinary bi-fundamental hypermultiplet transforming in the $(1,-1)$ representation of $\mathrm{U}(1) \times \mathrm{U}(1)$. An intuitive explanation of this is that a D3-brane extended between these two subwebs does not feel the presence of the orientifold and is oriented. In contrast, the distance between the upper right subweb and the lower-left subweb (mirror image to upper left) is given by $\left|m_{1}^{(7)}+m_{2}^{(7)}\right|$. This gives rise to an exotic hypermultiplet transforming as $(1,1)$ under $\mathrm{U}(1) \times \mathrm{U}(1)$. Since a D3-brane extending between these two subwebs must cross the orientifold, it is unoriented, which gives an intuitive explanation for the hypermultiplet's democratic nature. The number of each type of hypermultiplet follows, as is standard, by computing the stable intersection number. Altogether, this leads to the magnetic quiver shown in figure 12. This explains the appearance of the dashed lines in the second row of table 4. To make the proposal 


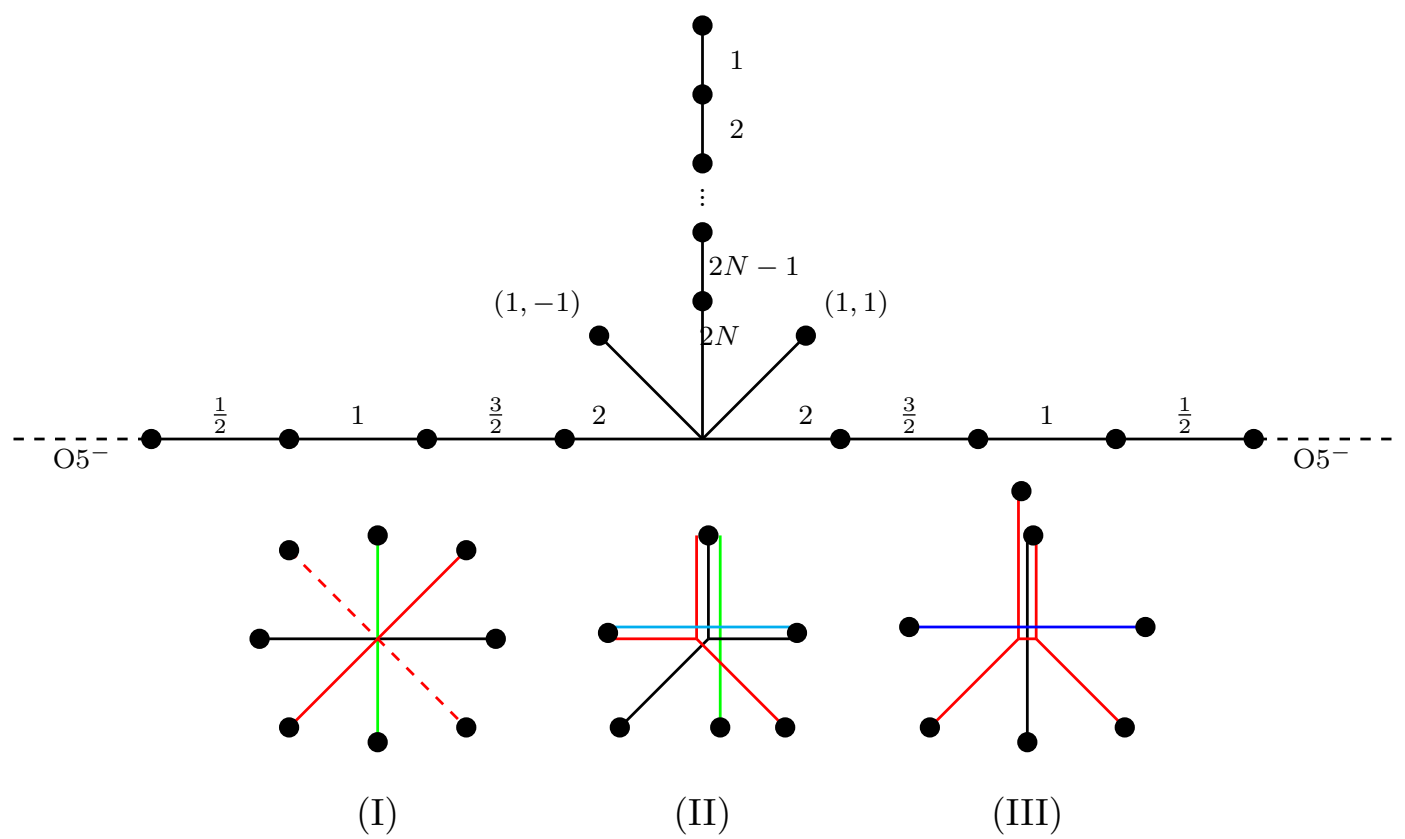

Figure 10. An orientifold web for the $\mathrm{Y}_{N}^{1,1}$ theory at the fixed point. We show the three possible maximal subdivisions of the centre of the junction at the bottom.

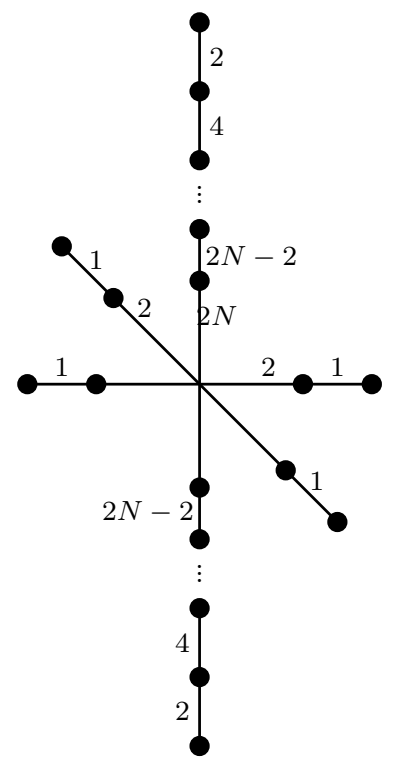

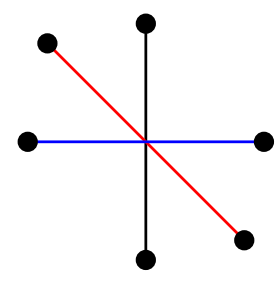

(I)

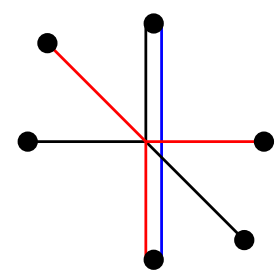

(III)

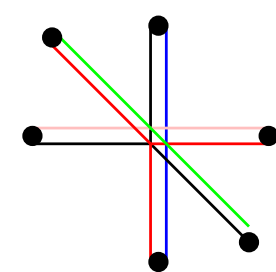

(II)

Figure 11. A unitary web for the $\mathrm{Y}_{N}^{1,1}$ theory at the fixed point, along with the three possible distinct maximal subdivisions of the centre of the junction.

more convincing, we compute the Coulomb branch Hilbert series for the OSp and unitary magnetic quivers. The results are collected in table 5, and they agree with each other. 


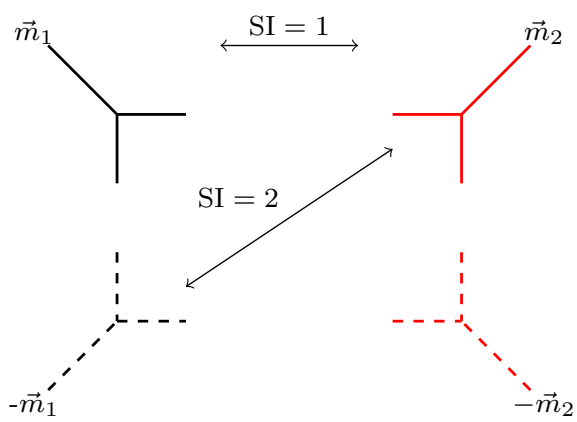

(a)

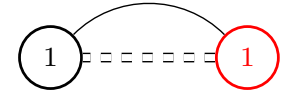

(b)

Figure 12. The origin of the exotic bi-fundamental hypermultiplets. (a) The maximal subdivision of the relevant web. The dashed lines correspond to the mirror images of the solid line subwebs. For ease of presentation we have not included the O5-plane in the picture. (b) The corresponding magnetic quiver with the exotic bi-fundamental hypermultiplet. 


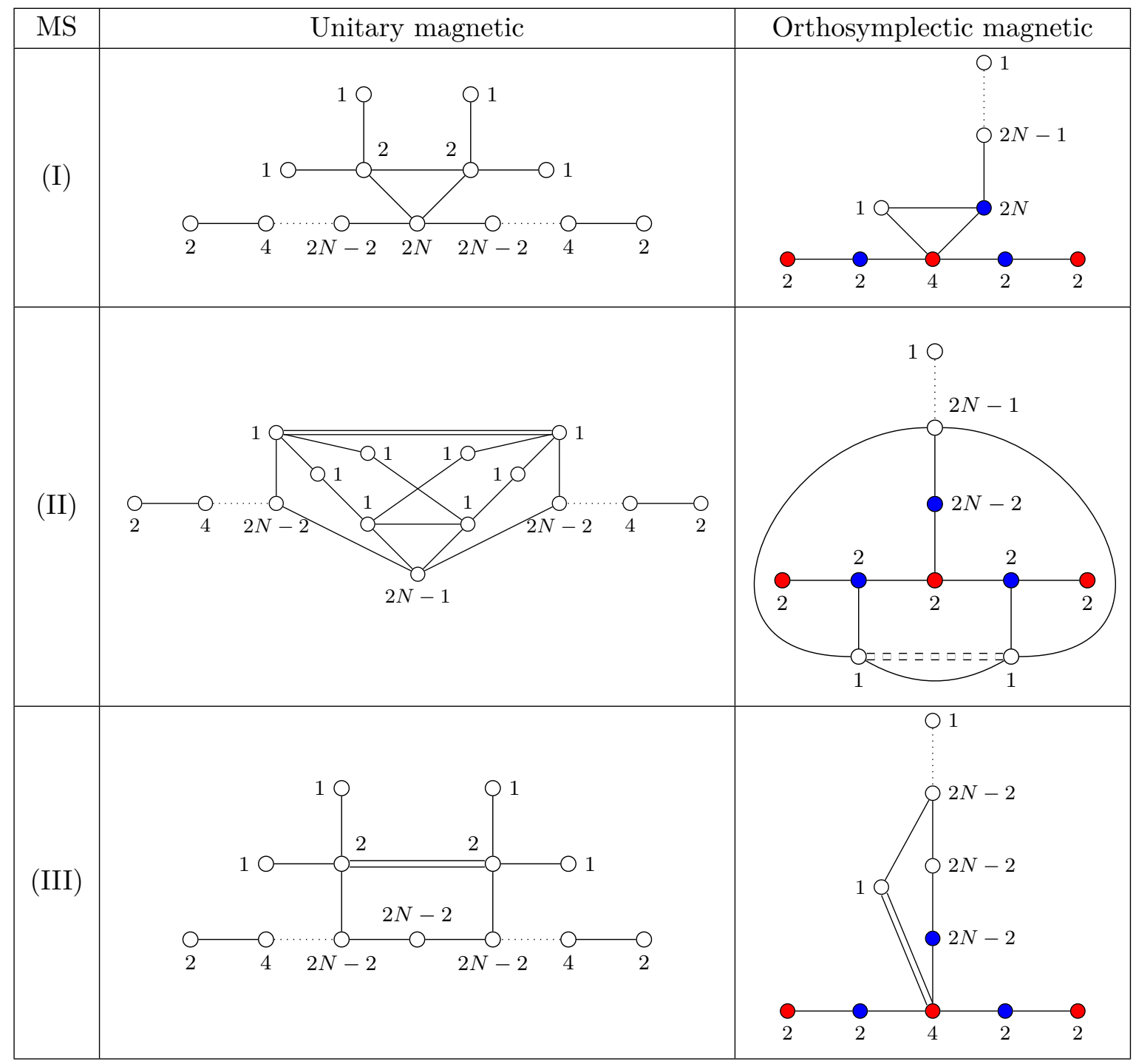

Table 4. The unitary and the orthosymplectic magnetic quivers derived from various maximal subdivisions (MS) corresponding to the unitary and the orientifold web diagrams in figure 11 and figure 10 respectively for the Higgs branch of $\mathrm{Y}_{N}^{1,1}$ theory. 


\begin{tabular}{|c|c|c|c|}
\hline \multirow{2}{*}{ MS } & Unitary magnetic quiver & \multicolumn{2}{|c|}{ Orthosymplectic magnetic quiver } \\
\hline & $\operatorname{HS}(t)$ & $\mathrm{HS}(t ; \vec{m} \in \mathbb{Z})$ & $\mathrm{HS}\left(t ; \vec{m} \in \mathbb{Z}+\frac{1}{2}\right)$ \\
\hline$(\mathrm{I})_{N=1}$ & $\begin{array}{c}1+16 t+185 t^{2}+1585 t^{3}+ \\
10919 t^{4}+62648 t^{5}+ \\
308937 t^{6}+1338676 t^{7}+ \\
5192925 t^{8}+18300090 t^{9}+ \\
59307538 t^{10}+\ldots\end{array}$ & $\begin{array}{c}1+16 t+153 t^{2}+1105 t^{3}+ \\
6759 t^{4}+35992 t^{5}+169449 t^{6}+ \\
713140 t^{7}+2714621 t^{8}+ \\
9447450 t^{9}+30359666 t^{10}+\ldots\end{array}$ & $\begin{array}{c}32 t^{2}+480 t^{3}+4160 t^{4}+ \\
26656 t^{5}+139488 t^{6}+ \\
625536 t^{7}+2478304 t^{8}+ \\
8852640 t^{9}+28947872 t^{10}+\ldots\end{array}$ \\
\hline$(\mathrm{I})_{N=2}$ & $\begin{array}{c}1+28 t+419 t^{2}+4519 t^{3}+ \\
39592 t^{4}+298310 t^{5}+\ldots\end{array}$ & $\begin{array}{c}1+28 t+419 t^{2}+4423 t^{3}+ \\
37000 t^{4}+261190 t^{5}+\ldots\end{array}$ & $96 t^{3}+2592 t^{4}+37120 t^{5}+\ldots$ \\
\hline$(\mathrm{II})_{N=1}$ & $\begin{array}{c}1+18 t+246 t^{2}+2266 t^{3}+ \\
15910 t^{4}+89506 t^{5}+ \\
422730 t^{6}+1728642 t^{7}+ \\
6272807 t^{8}+20573244 t^{9}+ \\
61888524 t^{10}+\ldots\end{array}$ & $\begin{array}{c}1+18 t+198 t^{2}+1530 t^{3}+ \\
9574 t^{4}+50466 t^{5}+ \\
229338 t^{6}+914946 t^{7}+ \\
3266279 t^{8}+10596380 t^{9}+ \\
31638956 t^{10}+\ldots\end{array}$ & $\begin{array}{c}48 t^{2}+736 t^{3}+6336 t^{4}+ \\
39040 t^{5}+193392 t^{6}+ \\
813696 t^{7}+3006528 t^{8}+ \\
9976864 t^{9}+30249568 t^{10}+\ldots\end{array}$ \\
\hline$(\mathrm{II})_{N=2}$ & $\begin{array}{c}1+30 t+476 t^{2}+5465 t^{3}+ \\
51395 t^{4}+416458 t^{5}+\ldots\end{array}$ & $\begin{array}{c}1+30 t+476 t^{2}+5305 t^{3}+ \\
46915 t^{4}+350474 t^{5}+\ldots\end{array}$ & $160 t^{3}+4480 t^{4}+65984 t^{5}+\ldots$ \\
\hline$(\mathrm{III})_{N=1}$ & $\begin{array}{l}\frac{P_{8}(t)}{(1-t)^{14}(1+t)^{7}} \\
=1+13 t+121 t^{2}+797 t^{3} \\
+4240 t^{4}+18760 t^{5}+\ldots\end{array}$ & $\begin{array}{l}\frac{P_{9}(t)}{(1-t)^{14}\left(1+t+t^{2}+t^{3}\right)^{7}} \\
=1+13 t+105 t^{2}+605 t^{3} \\
+2864 t^{4}+11640 t^{5}+\ldots\end{array}$ & $\begin{array}{l}\frac{P_{10}(t)}{(1-t)^{14}\left(1+t+t^{2}+t^{3}\right)^{7}} \\
=16 t^{2}+192 t^{3}+1376 t^{4} \\
+7120 t^{5}+\ldots\end{array}$ \\
\hline$(\mathrm{III})_{N=2}$ & $\begin{array}{c}1+28 t+419 t^{2}+4452 t^{3}+ \\
37756 t^{4}+270816 t^{5}+\ldots\end{array}$ & $\begin{array}{c}1+28 t+419 t^{2}+4388 t^{3}+ \\
36028 t^{4}+246496 t^{5}+\ldots\end{array}$ & $64 t^{3}+1728 t^{4}+24320 t^{5}+\ldots$ \\
\hline
\end{tabular}

Table 5. Coulomb branch Hilbert series of the unitary and the orthosymplectic magnetic quivers for different maximal subdivisions of the $\mathrm{Y}_{N}^{1,1}$ theory. The corresponding quivers are presented in table 4 . The explicit forms of $P_{8}(t), P_{9}(t), P_{10}(t)$ are given in appendix B. 


\subsubsection{The $\mathrm{Y}_{N}^{2 \times 1,2 \times 1}$ theory}

The examples we consider are brane configurations obtained by intersecting $2 N$ NS5, 2 $(1,1), 2(1,-1)$ and one D5 brane on top of an O5-plane which is asymptotically an $\mathrm{O}^{-}{ }^{-}$ plane. We call the theory on the web the $Y_{N}^{2 \times 1,2 \times 1}$ theory. At low energies the theory is described by the following quiver,

$$
[1 S+1 C]-\overbrace{\mathrm{SO}(6)-\mathrm{USp}(2)-\mathrm{SO}(6)-\cdots-\mathrm{USp}(2)-\mathrm{SO}(6)}^{2 N+1}-[1 S+1 C] .
$$

An orientifold web diagram is given in figure 19 .

It may also be understood as gluing $N-1$ copies of $\mathrm{SO}(6)$ with 2 vectors and 2 copies of $\mathrm{SO}(6)$ with one vector, one spinor, and one conjugate spinor, by successive gauging of USp(2) subgroups of the global symmetry. This latter viewpoint allows us to construct a unitary web for the same theory, by gluing $N-1$ copies of $\mathrm{SU}(4)_{0}$ with two second rank antisymmetric hypermultiplets, and 2 copies of SU(4) $)_{0}$ with 2 fundamentals and one 2 nd rank antisymmetric hypermultiplet, via gauging common $\mathrm{SU}(2)$ subgroups of the global symmetry. The construction is illustrated in figure 13. Using this method, we obtain a unitary web diagram for the theory of (2.16), and it is depicted in figure 20 .

At infinite coupling, there are 10 maximal subdivisions of the unitary web, of which we only show 8 explicitly in figure 20. Two further subdivisions are obtained, by 180-degree rotation of those labeled (V) and (VIII) in figure 20. The unitary magnetic quivers follow straightforwardly and are listed in the second column of table 6 . In the following, we provide a guide to extract the magnetic quivers from the orientifold web.

Consider the maximal subdivision (I) corresponding to the orientifold web in figure 19. The corresponding orthosymplectic magnetic quiver appears in table 6 . It is a fairly tame object, except for the appearance of the 2 antisymmetric hypermultiplets attached to one of the $\mathrm{U}(2)$ nodes. Figure 14 shows the subweb responsible for the $\mathrm{U}(2)$ gauge node. There are two $(1,1) 5$-branes, whose position in the transverse $x^{7,8,9}$ directions are denoted by $\vec{m}_{1}$ and $\vec{m}_{2}$ respectively. Consider a D3-brane which is suspended between the $(1,1)$ 5 -brane at position $m_{1}^{(7)}$, and the mirror of the $(1,1) 5$-brane at position $m_{2}^{(7)}$, which is located at position $-m_{2}^{(7)}$ along the $x^{7}$ direction. Clearly, the distance between the two subwebs is $\left|m_{1}^{(7)}+m_{2}^{(7)}\right|$, which is the weight corresponding to the second rank antisymmetric representation of $\mathrm{U}(2) .{ }^{5}$ The fact that there are two such multiplets follows, as is standard, from the stable intersection of the $(1,1)$ and $(1,-1) 5$-branes. One can repeat this exercise in the presence of $n(1,1) 5$-branes and their mirror images, and identify the weight system for the second rank antisymmetric representation of $\mathrm{U}(n)$ in a similar manner. To make this proposal more concrete, we computed the Coulomb and Higgs branch Hilbert series of the full orthosymplectic magnetic quiver corresponding to maximal subdivision (I) in

\footnotetext{
${ }^{5}$ In this specific case, the antisymmetric representation of $\mathrm{U}(2)$ is a singlet under the $\mathrm{SU}(2)$ factor and carries charge 2 under the U(1) factor of the gauge group. However, this should be distinguished from the rule on charge 2 hypermultiplets. For higher rank groups we expect a hypermultiplet transforming under the antisymmetric of $\mathrm{U}(N)$.
} 

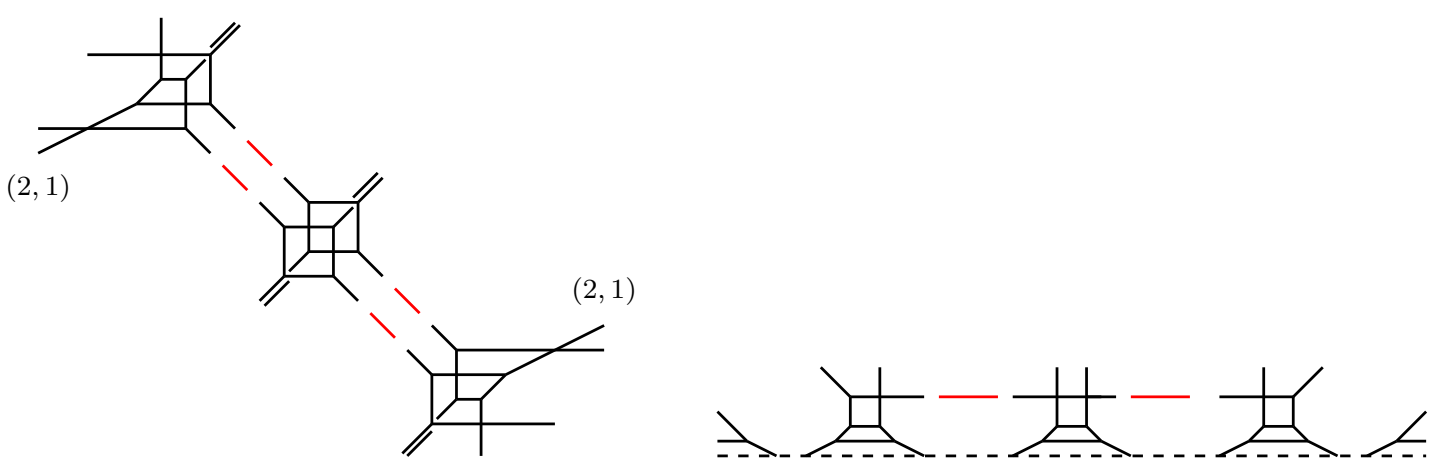

Figure 13. Constructing web diagrams for $\mathrm{Y}_{N}^{2 \times 1,2 \times 1}$ theory by gauging $\mathrm{SU}(2)$ 's.

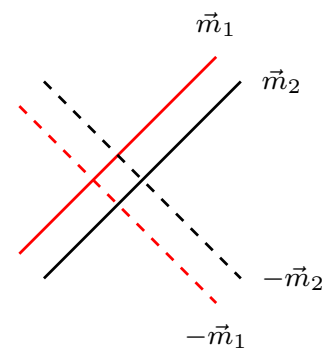

(a)

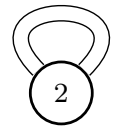

(b)

Figure 14. The origin of the antisymmetric matter appearing in some magnetic quivers.

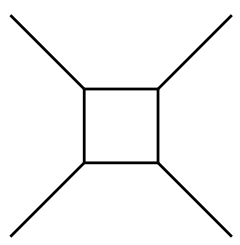

(a)

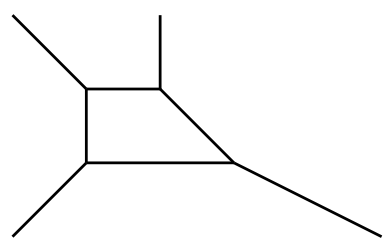

(b)

Figure 15. Usual 5-brane web diagram for (a) $E_{1}$ SCFT and (b) $\tilde{E}_{1}$ SCFT .

table 6 . The results are in agreement with the unitary magnetic quiver (see table 7 for the matching of the Coulomb branch Hilbert series).

Next, consider the maximal subdivision (III) of the orientifold web. Here, we encounter the first example of orientifold web diagrams with identical shapes, that are actually inequivalent. In order to clarify this situation, we start from a much simpler example, known as the rank $1 E_{1}$ SCFT and $\tilde{E}_{1}$ SCFT [4]. They correspond to the $5 \mathrm{~d} \mathcal{N}=1$ pure $\operatorname{SU}(2)$ gauge theories with discrete theta angle 0 and $\pi$, respectively. When we describe them in terms of the ordinary 5-brane web diagram, there are clear differences, as depicted in figure 15 [31]. This difference is interpreted as two inequivalent ways of decomposing an $O 7^{-}$-plane into two $(p, q)$ 7-branes [53]. 


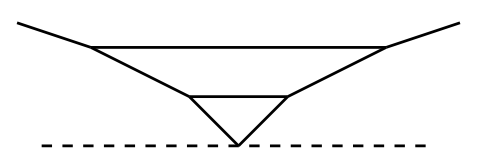

(a) with $\mathrm{O} 5$ for $E_{1} \mathrm{SCFT}$

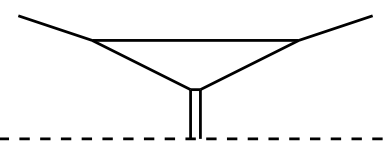

(b) with $\mathrm{O} 5$ for $\tilde{E}_{1} \mathrm{SCFT}$



(c) $E_{1}$ and $\tilde{E}_{1}$ theory at SCFT point

Figure 16. 5-brane web diagrams with O5-plane for $E_{1}$ and $\tilde{E}_{1}$ SCFTs.

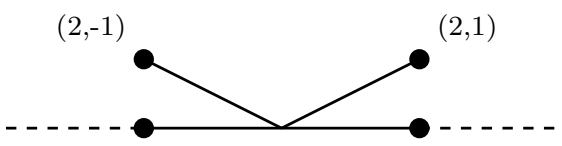

Figure 17. 5-brane web diagram with O5-plane for $E_{3} \mathrm{SCFT}$.

The situation in the 5-brane web diagram with O5-plane for these two theories is much subtler. These two web diagrams cannot be distinguished in the weakly coupled phase. However, there are clear difference in another phase as depicted in figure 16a and 16b [22]. This phase is the counterpart of the phase denoted in [31] as "past infinite coupling". These claims are justified from the analysis of the decompactification limit of the SeibergWitten curve obtained from the M5-brane configuration corresponding to these 5-brane webs with O5-plane.

Suppose that we start from these two different webs and go to SCFT point. Then, the difference disappears at the level of the 5-brane web diagram, as depicted in figure 16c. However, they should still be distinguished, taking into account that they correspond to two inequivalent 5d SCFT. That is, we should distinguish the 5-brane web diagram in figure 16c as a limit of figure 16a, from the 5-brane web diagram in figure 16c as a limit of figure 16b. Since we have already known that the former should give non-trivial Higgs branch while the latter should not give any continuous Higgs branch [40], we denote these two webs as "decomposable" web and "not decomposable" web, respectively. This discussion can be generalized to the web diagram where a $(p, 1)-5$ brane and its mirror image are intersecting on top of the $\mathrm{O}^{-}-$-plane for any $p$. This may be either decomposable to give non-trivial Higgs branch, or not decomposable to give no continuous Higgs branch.

As discussed in [1], we can see only one Higgs branch of the rank $1 E_{3}$ SCFT from naive analysis with the 5-brane web with O5-plane. However, once we accept the claims above, we can reproduce the two different branches of rank $1 E_{3}$ SCFT. Depending on whether the $(2,1) 5$-brane in figure 17 is either decomposable or not decomposable, they lead to two different magnetic quivers:

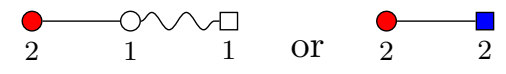

Here, not decomposable web, which cannot be detached from the O5-plane, contribute as a flavor. A different example of treating a subweb which cannot be detached from the O5-plane as a flavor is discussed in [1].

Analogous discussion is possible for still another type of 5-brane web. We should distinguish the diagram in figure $18 \mathrm{a}$ as a limit of the diagram in figure 18b, which is decomposable, and the diagram in figure 18a as a limit of the diagram in figure 18c, 


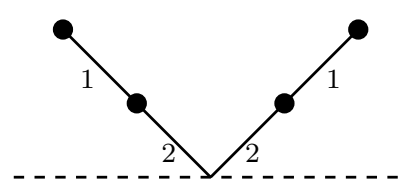

(a) A sub-web diagram

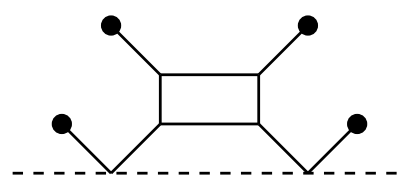

(b) Decomposable web

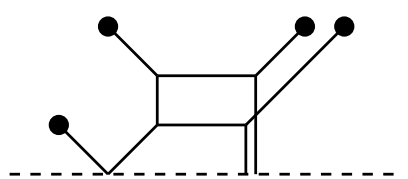

(c) Not decomposable web

Figure 18. Decomposable and not decomposable orientifold web diagrams in $Y_{N}^{2 \times 1,2 \times 1}$.

which is not decomposable. The former can be decomposed in a natural way to give $\mathrm{U}(1) \times \mathrm{U}(2) \times \mathrm{U}(1)$ gauge group in the magnetic quiver while the latter cannot be detached from the O5-plane and thus should be treated as a flavor. This claim is justified by comparing them with the equivalent ordinary 5 -brane web diagrams.

Now we go back to the $Y_{N}^{2 \times 1,2 \times 1}$ theory. The orientifold web in figure 19 includes the subweb in figure 18a. The maximal subdivision (I) includes the decomposable web, and the maximal subdivision (III) includes a subweb that is not decomposable.

Among the eight maximal subdivisions, the maximal subdivision (V) in figure 19 yields a magnetic quiver with a charge 2 hypermultiplet. The maximal subdivision (V) contains the subweb in red in the maximal subdivision in figure 19. We compute the number of the charge 2 hypermultiplets associated with the U(1) gauge node from the subweb by the rule in (2.6). The stable intersection number of the subweb with its mirror is

$$
\text { SI of subweb with its own mirror image }=10-2=8 \text {, }
$$

and the stable intersection number of the subweb with O5 is

$$
\text { SI of subweb with } \mathrm{O} 5=3 .
$$

Then the number of the charge 2 hypermultiplets from (2.6) is

$$
\frac{8}{2}-3=1
$$

for the $\mathrm{U}(1)$ node associated with the subweb in red of the maximal subdivision (V) in figure 19 .

With the rules described above, we propose the magnetic quivers from the orientifold web for the eight maximal subdivisions and the result is summarized in table 6 . We have checked the matching of the Coulomb branch Hilbert series of the unitary and orthosymplectic magnetic quivers which can be seen from table 7 .

There are a few more possible configurations in the $Y_{N}^{p, q}$ family. They do not give rise to any new rules, in addition to those already mentioned so far. They do however serve as working examples that demonstrate the validity of the rules proposed above. We refer the curious reader to appendix D. 


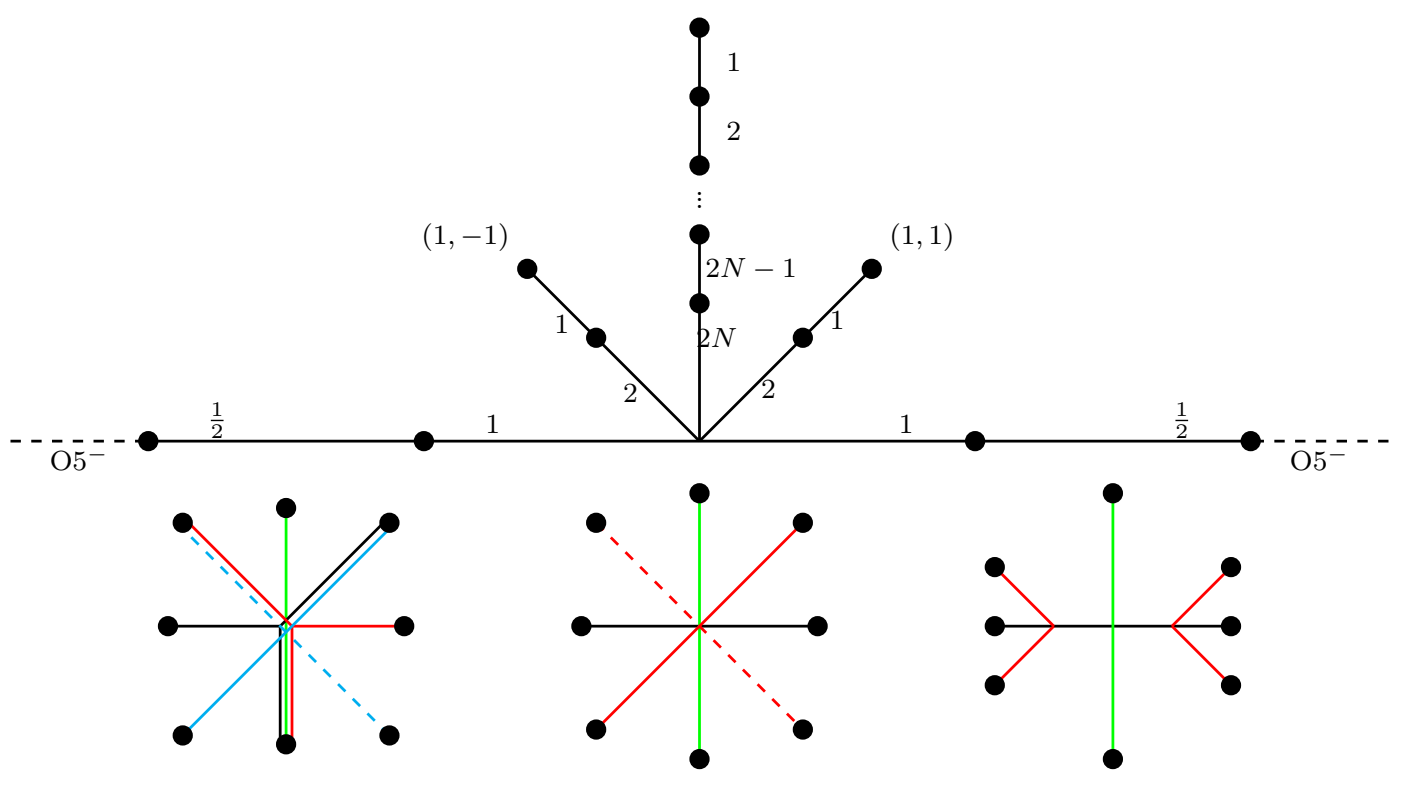

(II)

(I)

(III)

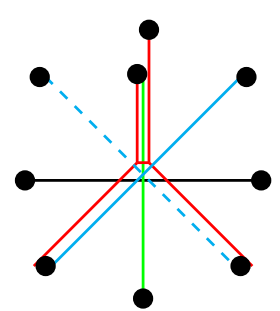

(IV)

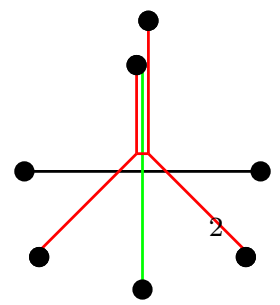

(VII) $N \geq 2$



(VIII) $N \geq 2$

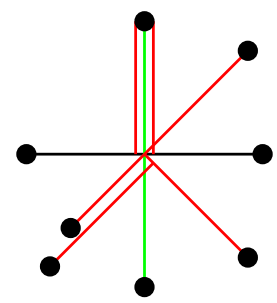

(V)

Figure 19. An orientifold web for the $Y_{N}^{2 \times 1,2 \times 1}$ theory along with the possible maximal subdivisions at the centre of the junction. 




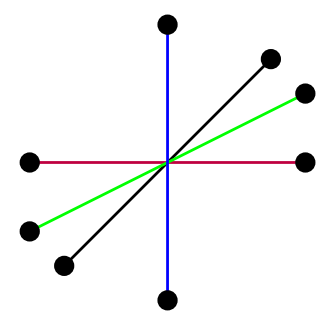

(I)

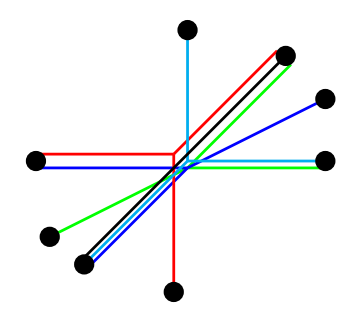

(IV)



(VII)



(II)



(V)

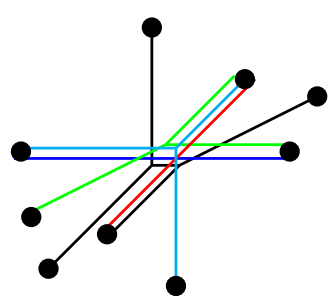

(VIII)

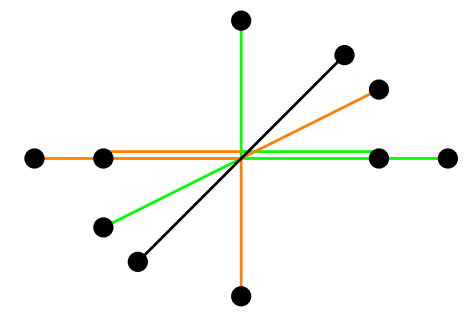

(III)

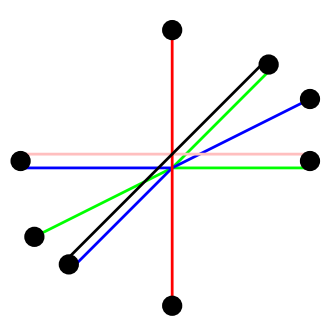

(VI) 


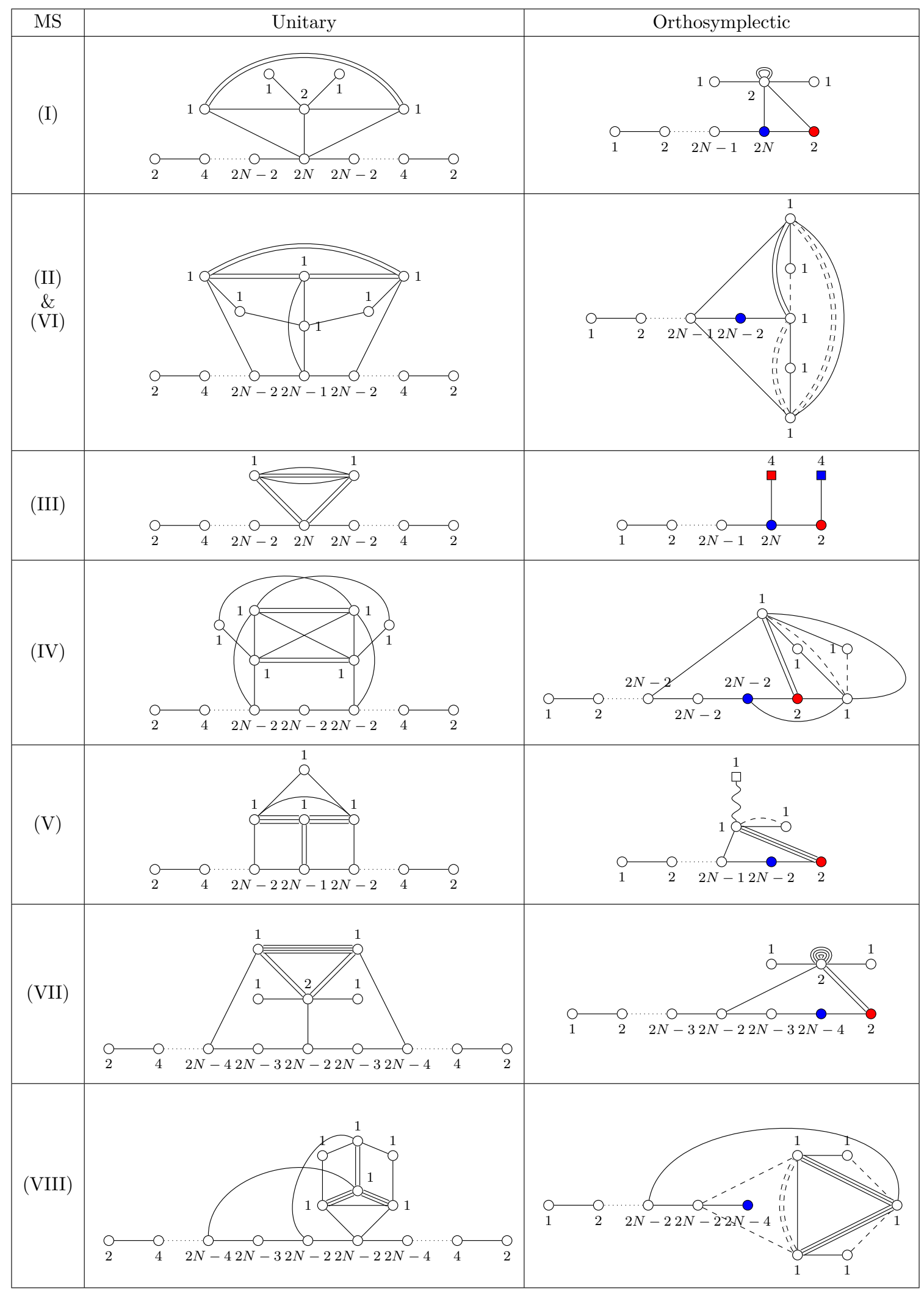

Table 6. Magnetic quivers for various maximal subdivisions (MS) of $Y_{N}^{2 \times 1,2 \times 1}$ theory. 


\begin{tabular}{|c|c|c|c|}
\hline \multirow{2}{*}{ MS } & Unitary & \multicolumn{2}{|c|}{ Orthosymplectic } \\
\hline & $\operatorname{HS}(t)$ & $\mathrm{HS}(t ; \vec{m} \in \mathbb{Z})$ & $\mathrm{HS}\left(t ; \vec{m} \in \mathbb{Z}+\frac{1}{2}\right)$ \\
\hline$(\mathrm{I})_{N=0}$ & $\begin{array}{l}\frac{P_{11}(t)}{(1-t)^{10}(1+t)^{5}} \\
=1+16 t+132 t^{2}+735 t^{3} \\
+3134 t^{4}+10974 t^{5}+\ldots\end{array}$ & $\begin{array}{l}\frac{P_{12}(t)}{(1-t)^{10}(1+t)^{5}\left(1+t^{2}\right)} \\
=1+8 t+72 t^{2}+371 t^{3} \\
+1598 t^{4}+5510 t^{5}+\ldots\end{array}$ & $\begin{array}{l}\frac{P_{13}(t)}{(1-t)^{10}(1+t)^{5}\left(1+t^{2}\right)} \\
=8 t+60 t^{2}+364 t^{3} \\
+1536 t^{4}+5464 t^{5}+\ldots\end{array}$ \\
\hline$(\mathrm{I})_{N=1}$ & $\begin{array}{c}1+11 t+84 t^{2}+485 t^{3}+ \\
2346 t^{4}+9738 t^{5}+\cdots\end{array}$ & $\begin{array}{c}1+11 t+68 t^{2}+317 t^{3}+ \\
1346 t^{4}+5290 t^{5}+\cdots\end{array}$ & $\begin{array}{c}16 t^{2}+168 t^{3}+1000 t^{4}+ \\
4448 t^{5}+\cdots\end{array}$ \\
\hline$(\mathrm{I})_{N=2}$ & $\begin{array}{c}1+23 t+290 t^{2}+2653 t^{3}+ \\
19602 t^{4}+123630 t^{5}+\ldots\end{array}$ & $\begin{array}{c}1+23 t+290 t^{2}+2605 t^{3}+ \\
18522 t^{4}+110470 t^{5}+\ldots\end{array}$ & $48 t^{3}+1080 t^{4}+13160 t^{5}+\ldots$ \\
\hline $\begin{array}{l}(\mathrm{II})_{N=1} \\
\& \\
(\mathrm{VI})_{N=1}\end{array}$ & $\begin{array}{c}1+12 t+91 t^{2}+8 t^{5 / 2}+ \\
484 t^{3}+104 t^{7 / 2}+2032 t^{4}+ \\
720 t^{9 / 2}+7152 t^{5}+\cdots\end{array}$ & $\begin{array}{c}1+12 t+75 t^{2}+336 t^{3}+ \\
16 t^{7 / 2}+1268 t^{4}+208 t^{9 / 2}+ \\
4220 t^{5}+\cdots\end{array}$ & $\begin{array}{l}16 t^{2}+8 t^{5 / 2}+148 t^{3}+88 t^{7 / 2}+ \\
764 t^{4}+512 t^{9 / 2}+2932 t^{5}+\cdots\end{array}$ \\
\hline $\begin{array}{l}(\mathrm{II})_{N=2} \\
\& \\
(\mathrm{VI})_{N=2}\end{array}$ & $\begin{array}{c}1+24 t+313 t^{2}+2943 t^{3}+ \\
32 t^{7 / 2}+22157 t^{4}+ \\
768 t^{9 / 2}+140921 t^{5}+\cdots\end{array}$ & $\begin{array}{c}1+24 t+313 t^{2}+2895 t^{3}+ \\
21089 t^{4}+32 t^{9 / 2}+ \\
128073 t^{5}+\cdots\end{array}$ & $\begin{array}{c}48 t^{3}+32 t^{7 / 2}+1068 t^{4}+ \\
736 t^{9 / 2}+12848 t^{5}+\cdots\end{array}$ \\
\hline$(\mathrm{III})_{N=1}$ & $\begin{array}{l}\frac{P_{14}(t)}{(1-t)^{2}\left(1-t^{3}\right)\left(1-t^{4}\right)^{3}} \\
=1+4 t+13 t^{2}+33 t^{3} \\
+80 t^{4}+165 t^{5}+\cdots\end{array}$ & $\begin{array}{l}\frac{P_{14}(t)}{(1-t)^{2}\left(1-t^{3}\right)\left(1-t^{4}\right)^{3}} \\
=1+4 t+13 t^{2}+33 t^{3} \\
+80 t^{4}+165 t^{5}+\cdots\end{array}$ & not required \\
\hline$(\mathrm{III})_{N=2}$ & $\begin{array}{c}1+16 t+151 t^{2}+1039 t^{3}+ \\
5750 t^{4}+26954 t^{5}+\cdots\end{array}$ & $\begin{array}{c}1+16 t+151 t^{2}+1039 t^{3}+ \\
5750 t^{4}+26954 t^{5}+\cdots\end{array}$ & not required \\
\hline$(\mathrm{IV})_{N=1}$ & $\begin{array}{c}1+9 t+43 t^{2}+16 t^{5 / 2}+ \\
157 t^{3}+128 t^{7 / 2}+488 t^{4}+ \\
560 t^{9 / 2}+1400 t^{5}+\cdots\end{array}$ & $\begin{array}{c}1+9 t+43 t^{2}+157 t^{3}+ \\
488 t^{4}+1400 t^{5}+\cdots\end{array}$ & $\begin{array}{c}16 t^{5 / 2}+128 t^{7 / 2}+ \\
560 t^{9 / 2}+\cdots\end{array}$ \\
\hline$(\mathrm{IV})_{N=2}$ & $\begin{array}{c}1+24 t+313 t^{2}+2860 t^{3}+ \\
64 t^{7 / 2}+20297 t^{4}+ \\
1472 t^{9 / 2}+118722 t^{5}+\cdots \\
\end{array}$ & $\begin{array}{c}1+24 t+313 t^{2}+2860 t^{3}+ \\
20297 t^{4}+118722 t^{5}+\cdots\end{array}$ & $64 t^{7 / 2}+1472 t^{9 / 2}+\cdots$ \\
\hline$(\mathrm{V})_{N=1}$ & $\begin{array}{l}\frac{P_{15}(t)}{(1-t)^{8}\left(1-t^{3}\right)\left(1-t^{5}\right)^{3}} \\
=1+8 t+34 t^{2}+8 t^{5 / 2} \\
+106 t^{3}+56 t^{7 / 2}+275 t^{4} \\
+216 t^{9 / 2}+646 t^{5}+\cdots\end{array}$ & $\begin{array}{l}\frac{P_{16}(t)}{(1-t)^{8}\left(1-t^{3}\right)\left(1-t^{10}\right)^{3}} \\
=1+8 t+34 t^{2}+106 t^{3} \\
+275 t^{4}+646 t^{5}+\cdots\end{array}$ & $\begin{array}{l}\frac{8 t^{5 / 2} P_{17}(t)}{(1-t)^{8}\left(1-t^{3}\right)\left(1-t^{10}\right)^{3}} \\
=8 t^{5 / 2}+56 t^{7 / 2} \\
+216 t^{9 / 2}+\cdots\end{array}$ \\
\hline$(\mathrm{V})_{N=2}$ & $\begin{array}{c}1+20 t+224 t^{2}+1803 t^{3}+ \\
32 t^{7 / 2}+11510 t^{4}+ \\
608 t^{9 / 2}+61468 t^{5}+\cdots \\
\end{array}$ & $\begin{array}{c}1+20 t+224 t^{2}+1803 t^{3}+ \\
11510 t^{4}+61468 t^{5}+\cdots\end{array}$ & $32 t^{7 / 2}+608 t^{9 / 2}+\cdots$ \\
\hline$(\mathrm{VII})_{N=2}$ & $\begin{array}{c}1+23 t+259 t^{2}+1992 t^{3}+ \\
11927 t^{4}+59343 t^{5}+\cdots\end{array}$ & $\begin{array}{c}1+23 t+259 t^{2}+1944 t^{3}+ \\
11075 t^{4}+51523 t^{5}+\cdots\end{array}$ & $48 t^{3}+852 t^{4}+7820 t^{5}+\cdots$ \\
\hline$(\mathrm{VII})_{N=3}$ & $\begin{array}{c}1+43 t+980 t^{2}+15615 t^{3}+ \\
194028 t^{4}+\cdots\end{array}$ & $\begin{array}{c}1+43 t+980 t^{2}+15615 t^{3}+ \\
193868 t^{4}+\cdots\end{array}$ & $160 t^{4}+\cdots$ \\
\hline$(\mathrm{VIII})_{N=2}$ & $\begin{array}{c}1+24 t+297 t^{2}+2560 t^{3}+ \\
32 t^{7 / 2}+17229 t^{4}+ \\
736 t^{9 / 2}+95960 t^{5}+\cdots \\
\end{array}$ & $\begin{array}{c}1+24 t+297 t^{2}+2512 t^{3}+ \\
16299 t^{4}+32 t^{9 / 2}+ \\
86552 t^{5}+\cdots \\
\end{array}$ & $\begin{array}{c}48 t^{3}+32 t^{7 / 2}+930 t^{4}+ \\
704 t^{9 / 2}+9408 t^{5}+\cdots\end{array}$ \\
\hline
\end{tabular}

Table 7. Coulomb branch Hilbert series of the unitary and orthosymplectic magnetic quivers for different maximal subdivisions (MS) of $Y_{N}^{2 \times 1,2 \times 1}$ theory. The corresponding quivers are presented in table 6 . The explicit forms of $P_{11}(t), \cdots, P_{17}(t)$ are given in appendix B. 


\subsection{The $H_{N}$ family}

The final example in section 2 arises from decoupling flavors from the unitary electric quiver for the $\#_{3, N}$ theory $(2.3)$. We denote by $H_{N}^{p, q}$, the theory obtained by decoupling flavors attached to the central node in (2.3). Here $p(q)$ are the number of flavors integrated out with positive (negative) mass such that $M=2 N-p-q$. In other words, the $H_{N}^{p, q}$ theory is nothing but

$$
\begin{gathered}
{[M]} \\
\mid \\
\mathrm{SU}(N)-\mathrm{SU}(2 N)_{\frac{p-q}{2}}-\mathrm{SU}(N)
\end{gathered} .
$$

The unitary and orientifold web diagram for the $H_{N}^{p, q}$ theory is obtained from those of $+_{3, N}$ theory, i.e. figure 3 and figure 1 respectively. Note that in the orientifold web of figure 1 , the desirable mass deformation corresponds to the position of the external NS5 branes along the horizontal axis, a fact which is more transparent in the S-dual frame. Thus the orientifold web description of the $H_{N}^{p, q}$ theory is obtained from that of $+_{3, N}$ theory by decoupling, say, $p$ of the external NS5 branes to the left, and $q$ to the right.

Let us focus on the case with $p=q=N$. The 5 d theory (2.21) becomes

$$
\mathrm{SU}(N)-\mathrm{SU}(2 N)_{0}-\mathrm{SU}(N)
$$

When $N=1$, the $5 \mathrm{~d}$ theory is simply the $\mathrm{SU}(2)$ gauge theory with four flavors. The orientifold web diagram and the unitary web diagram at the infinitely strong coupling of the theory (2.22) are depicted in figure 21 and figure 22 respectively. An interesting point about the orientifold web diagram in figure 21 is that the diagram has $(1, N)$ and $(1,-N)$ 5-branes where $N$ can be larger than 1 and they intersect on the orientifold plane. This is a new feature which has not appeared in the past web diagrams. Hence this example is important for checking the rule (2.6) for the number of charge 2 hypermultiplets attached to the $\mathrm{U}(1)$ gauge node originated from the $(1, N)$ and $(1,-N)$ 5-branes with $N \geq 2 .{ }^{6}$ From the web diagrams it is possible to infer the corresponding magnetic quiver theories and we argue that they are the ones given in table 8 . Note that the number of the charge 2 hypermultiplets attached to the $\mathrm{U}(1)$ gauge node in the orthosymplectic magnetic quiver is zero due to (2.6). Indeed with this number for the charge 2 hypermultiplets we find perfect agreement between the Coulomb branch Hilbert series of the unitary and orthosymplectic magnetic quiver theories. We summarize the Coulomb branch Hilbert series of the magnetic quivers in table 8 for various $N$ in table 9 .

\footnotetext{
${ }^{6}$ We will consider cases which involve $(p, q)$ and $(p,-q)$ 5-branes with both $p$ and $q$ larger than 1 in appendix C.
} 


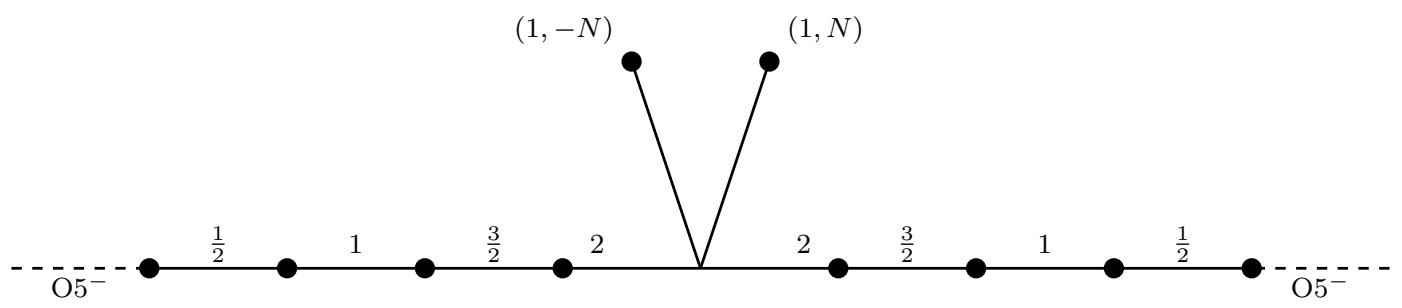

Figure 21. The orientifold web diagram for the $H_{N}^{N, N}$ theory at the infinitely strong coupling.

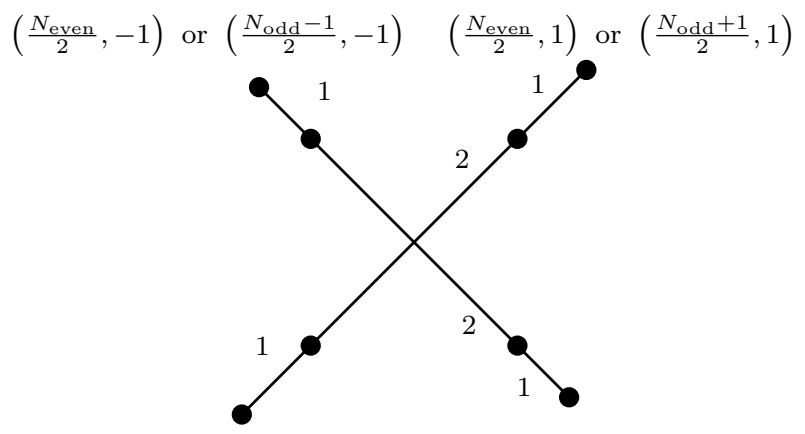

Figure 22. The unitary web diagram for the $H_{N}^{N, N}$ theory at the infinitely strong coupling.

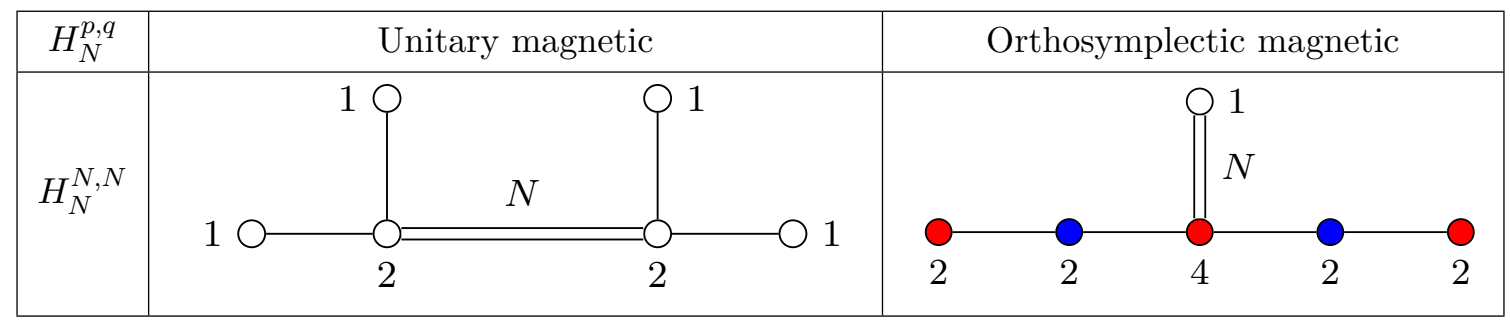

Table 8. Magnetic quivers for the $H_{N}^{N, N}$ family. The index $N$ in each figure denotes the number of the hypermultiplets.

\begin{tabular}{|c|c|c|c|}
\hline \multirow{2}{*}{$H_{N}^{N, N}$} & Unitary magnetic quiver & \multicolumn{2}{|c|}{ Orthosymplectic magnetic quiver } \\
\cline { 2 - 4 } & $\mathrm{HS}(t)$ & $\operatorname{HS}(t ; \vec{m} \in \mathbb{Z})$ & $\operatorname{HS}\left(t ; \vec{m} \in \mathbb{Z}+\frac{1}{2}\right)$ \\
\hline$H_{2}^{2,2}$ & $\begin{array}{c}1+13 t+121 t^{2}+797 t^{3}+ \\
4240 t^{4}+18760 t^{5}+\cdots\end{array}$ & $\begin{array}{c}1+13 t+105 t^{2}+605 t^{3}+ \\
2864 t^{4}+11640 t^{5}+\cdots\end{array}$ & $\begin{array}{c}16 t^{2}+192 t^{3}+1376 t^{4}+ \\
7120 t^{5}+\cdots\end{array}$ \\
\hline$H_{3}^{3,3}$ & $\begin{array}{c}1+13 t+89 t^{2}+461 t^{3}+ \\
2007 t^{4}+7579 t^{5}+\cdots\end{array}$ & $\begin{array}{c}1+13 t+89 t^{2}+445 t^{3}+ \\
1815 t^{4}+6347 t^{5}+\cdots\end{array}$ & $16 t^{3}+192 t^{4}+1232 t^{5}+\cdots$ \\
\hline$H_{4}^{4.4}$ & $\begin{array}{c}1+13 t+89 t^{2}+429 t^{3}+ \\
1671 t^{4}+5659 t^{5}+\cdots\end{array}$ & $\begin{array}{c}1+13 t+89 t^{2}+429 t^{3}+ \\
1655 t^{4}+5467 t^{5}+\cdots\end{array}$ & $16 t^{4}+192 t^{5}+\cdots$ \\
\hline$H_{5}^{5,5}$ & $\begin{array}{c}1+13 t+89 t^{2}+429 t^{3}+ \\
1639 t^{4}+5323 t^{5}+\cdots\end{array}$ & $\begin{array}{c}1+13 t+89 t^{2}+429 t^{3}+ \\
1639 t^{4}+5307 t^{5}+\cdots\end{array}$ & $16 t^{5}+\cdots$ \\
\hline
\end{tabular}

Table 9. Coulomb branch Hilbert series of the unitary and orthosymplectic magnetic quivers for the $H_{N}^{N, N}$ family whose quivers are presented in table 8 . 


\section{Magnetic quivers from $\mathrm{O5}^{+}-\mathrm{O}^{+}$}

Next we consider examples which arise from brane configurations with asymptotic $\mathrm{O} 5^{+}-$ planes on both the ends.

\subsection{The $+_{1, N}$ theory}

Intersecting $2 N$ NS5s, and a single D5 on top of on orientifold plane that is asymptotically an $\mathrm{O} 5^{+}$-plane, we arrive at the $+_{1, N}$ theory (figure 23). It has an IR gauge theory description as

$$
[1]-\overbrace{\mathrm{SO}(6)-\mathrm{USp}(2)-\mathrm{SO}(6)-\cdots-\mathrm{USp}(2)-\mathrm{SO}(6)}^{2 N-1}-[1] .
$$

It can also be understood as gluing $N$ copies of $\mathrm{SO}(6)$ with two vector hypermultiplets by successive gauging of $\operatorname{USp}(2)$ subgroups of the flavour symmetry. One can therefore engineer the same theory with an ordinary web diagram by gluing together $N$ copies of $\mathrm{SU}(4)_{0}$ with 2 antisymmetric hypermultiplets, via successive gauging of $\mathrm{SU}(2)$ subgroups of the global symmetry (figure 24).

In this setup, the $2 N$ NS5-branes intersecting with the $\mathrm{O}^{+}$-plane contribute to the magnetic quiver as a $\operatorname{USp}(2 N)$ gauge node. Here, we claim that there is a new feature in this case, which did not appear for NS5-branes intersecting with $\mathrm{O}^{-}$-plane. In order for the orthosymplectic magnetic quiver to give consistent results with the corresponding unitary quiver, we find that we need to add three fundamental half-hypermultiplets on this $\mathrm{USp}(2 N)$ gauge node.

We would like to interpret these three half-hypermultiplets as follows. First, we observe that the $\mathrm{RR}$ charge of the $\mathrm{O} 5^{+}$-plane is identical to the sum of the $\mathrm{RR}$ charges of $\mathrm{O} 5^{-}$-plane and of four half D5-branes. This motivates us to treat $\mathrm{O} 5^{+}$-plane as if it is the composite of them:

$$
\left(\mathrm{O} 5^{+} \text {-plane }\right)=\left(\mathrm{O} 5^{-} \text {-plane }\right)+4 \times(\text { Half D5 branes }) .
$$

Here, we assume that the half D5 branes cannot be detached from the O5--plane. Basically, the charge 1 hypermultiplet can be reinterpreted as coming from the D3-branes suspended between the NS5-branes and these half D5-branes. However, we need a further explanation of why the number of half-hypermultiplets is three instead of four. In this setup, there are two half D5-branes on top of the $05^{+}$-plane, producing $\mathrm{SO}(3)$ gauge group in the magnetic quiver. We would like to interpret that one out of the four half D5-branes inside the $\mathrm{O}^{+}$-plane is used for constructing an $\mathrm{SO}(3)$ gauge group. In other words, the D3brane suspended between the NS5-branes and this half D5-brane contributes as a part of the bi-fundamental hypermultiplets between the $\operatorname{USp}(2 N)$ node and the $\mathrm{SO}(3)$ node. This indicates that only the remaining three out of the four half D5-branes contribute as fundamental half-hypermultiplets.

Under this assumption, we find the agreement between the Hilbert series of the orthosymplectic quiver in figure $25 \mathrm{~b}$ and the Hilbert series of the unitary quiver in figure 25a 


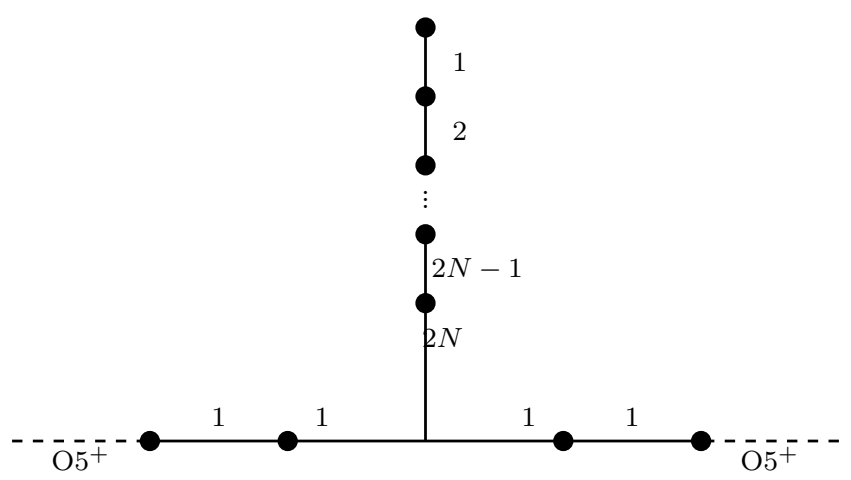

Figure 23. An orientifold web of the $+_{1, N}$ theory with asymptotically $\mathrm{O} 5^{+}$orientifold planes at strong coupling.

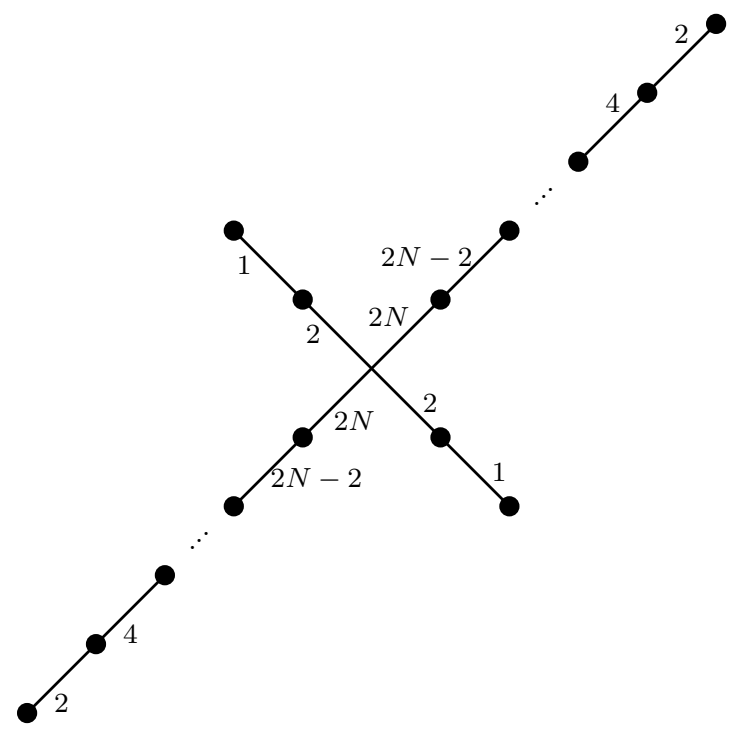

Figure 24. A unitary web for the $+_{1, N}$ theory with asymptotically $\mathrm{O} 5^{+}$orientifold planes.

both for the Coulomb branches and for the Higgs branches. In particular, the Higgs branch Hilbert series matching is crucial to settle the question about whether the $\mathrm{O}(1) \simeq \mathbb{Z}_{2}$ nodes in the orthosymplectic quiver are flavor or gauge nodes. Such a match is only obtained if $\mathbb{Z}_{2}$ gaugings are assumed. The obtained result is:

$$
\mathrm{HS}_{\mathbb{H}}(t)=1+3 t^{2}+15 t^{4}+36 t^{6}+98 t^{8}+\cdots
$$

The results of the Coulomb branch Hilbert series is tabulated in table 10 .

Encouraged by this agreement, we propose the following rule for the $\operatorname{USp}(2 N)$ gauge node coming from the $2 N$ NS5-branes intersecting with $\mathrm{O} 5^{+}$-plane. If this $\mathrm{USp}(2 N)$ gauge node has a bi-fundamental hypermultiplet with the $\mathrm{SO}$ (odd) gauge node coming from the D5-branes on the $\mathrm{O} 5^{+}$-plane, there are three fundamental half-hypermultiplets. Otherwise, there are four fundamental half-hypermultiplets. 


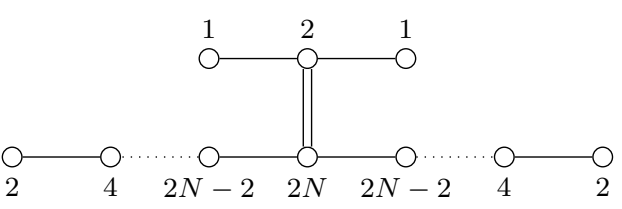

(a) Unitary quiver



(b) Orthosymplectic quiver

Figure 25. Magnetic quivers for the $+_{1, N}$ theory

\begin{tabular}{|c|c|c|c|}
\hline \multirow{2}{*}{$++_{1, N}$} & Unitary magnetic quiver & \multicolumn{2}{|c|}{ Orthosymplectic magnetic quiver } \\
\cline { 2 - 4 } & $\operatorname{HS}(t)$ & $\operatorname{HS}(t ; \vec{m} \in \mathbb{Z})$ & $\operatorname{HS}\left(t ; \vec{m} \in \mathbb{Z}+\frac{1}{2}\right)$ \\
\hline \multirow{4}{*}{$+_{1,1}$} & $\frac{P_{18}(t)}{(1-t)^{10}(1+t)^{5}}$ & $\frac{P_{18}(t)}{(1-t)^{10}(1+t)^{5}}$ & \\
& $=1+13 t+100 t^{2}+527 t^{3}$ & $1+13 t+100 t^{2}+527 t^{3}$ & not required \\
& $+2174 t^{4}+7425 t^{5}+\cdots$ & $+2174 t^{4}+7425 t^{5}+\cdots$ & \\
\hline \multirow{2}{*}{$+_{1,2}$} & $1+21 t+249 t^{2}+2188 t^{3}+$ & $1+21 t+249 t^{2}+2188 t^{3}+$ & \multirow{2}{*}{ not required } \\
\hline
\end{tabular}

Table 10. Coulomb branch Hilbert series for the unitary and orthosymplectic magnetic quivers in figure $25 \mathrm{a}$ and figure $25 \mathrm{~b}$ for the $+_{1, N}$ theory. The explicit form of $P_{18}(t)$ is given in appendix B.

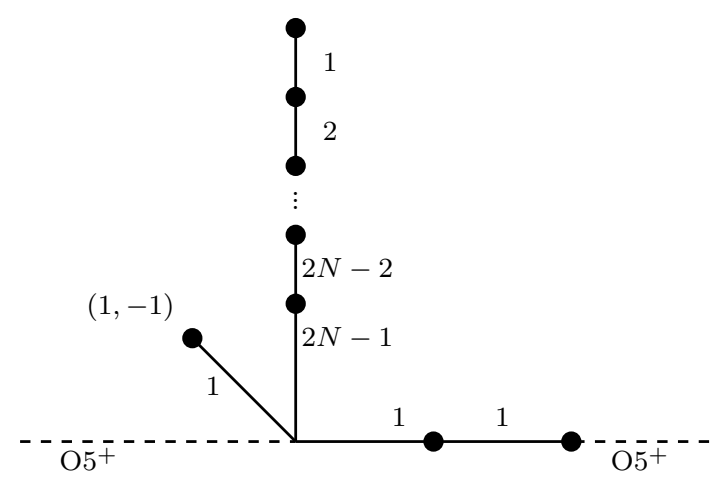

Figure 26. An orientifold web for the $\hat{K}_{N}^{1}$ theory with asymptotically $\mathrm{O} 5^{+}$planes

\subsection{The $\hat{K}_{N}^{1}$ theory}

Decoupling a single flavor from, say, the leftmost node in the $+_{1, N}$ theory (3.1), one arrives at the $\hat{K}_{N}^{1}$ theory. It has an IR gauge theory description as

$$
\overbrace{\mathrm{SO}(6)-\mathrm{USp}(2)-\mathrm{SO}(6)-\cdots-\mathrm{USp}(2)-\mathrm{SO}(6)-[1]}^{2 N-1}[1]
$$

We depict the orientifold and unitary web in figure 26 and figure 27 respectively. 


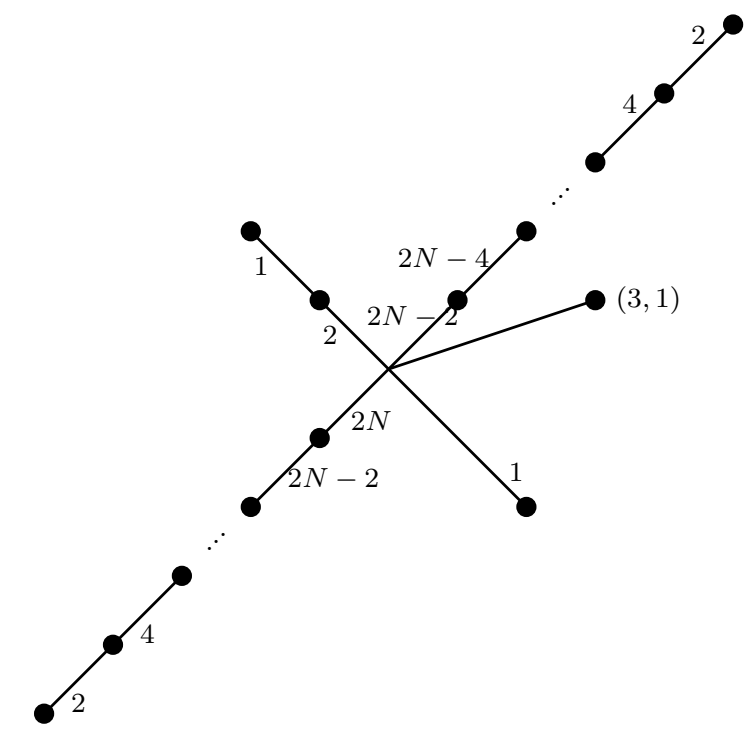

Figure 27. A unitary web for the $\hat{K}_{N}^{1}$ theory with asymptotically $\mathrm{O} 5^{+}$orientifold planes.

Also, in this setup, we find $\operatorname{USp}(2 N-2)$ gauge node coming from the $2 N-2$ NS5-branes intersecting with the $\mathrm{O} 5^{+}$-plane. According to the proposal in the previous subsection, there are four fundamental half hypermultiplets for this node because there is no SO(odd) gauge node coupled to this $\operatorname{USp}(2 N-2)$ gauge node.

In addition, there is one subweb intersecting with the $\mathrm{O} 5^{+}$-plane, contributing as a $\mathrm{U}(1)$ gauge node of the magnetic quiver. In general, when a subweb is intersecting with the $\mathrm{O} 5^{+}$-plane, it would be reasonable to expect that there are contributions from the D3-branes suspended between the considered subweb and its mirror image. Analogous to the case with $\mathrm{O} 5^{-}$-plane, such contribution is the hypermultiplets with charge 2 coupled to the corresponding $\mathrm{U}(1)$ node. The number of such charge 2 hypermultiplets would be given schematically by

$$
\frac{(\text { SI with its mirror image })}{2}-\left(\text { SI with } \mathrm{O}^{+}\right),
$$

as discussed around (2.6). In this specific setup, the number of the charge 2 hypermultiplets should be zero, in order to agree with the unitary magnetic quiver.

However, again, the situation for such $\mathrm{U}(1)$ node is different from the case with $\mathrm{O}^{-}$plane. Analogous to the case with NS5-branes intersecting with the $\mathrm{O} 5^{+}$-plane, the idea discussed around (3.2) implies that there is a contribution from the D3-branes suspended between this subweb and the half D5-branes included in the $\mathrm{O} 5^{+}$-plane. Such D3-branes correspond to hypermultiplets with charge 1 instead of charge 2 because the distance between the considered subweb and the $\mathrm{O} 5^{+}$-plane is half the distance between the original subweb and its mirror image. In order for the orthosymplectic magnetic quiver to be consistent with the unitary quiver, we need three hypermultiplets with charge 1 coupled to the $\mathrm{U}(1)$ node. This three is interpreted as

4(bare SI with half D5) - (Contribution from the common half D7 on the $\left.\mathrm{O} 5^{+}\right)$. 


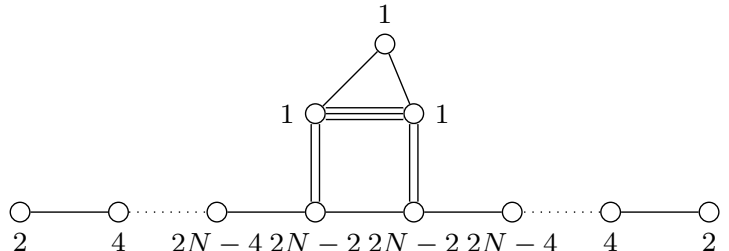

(a) Unitary quiver

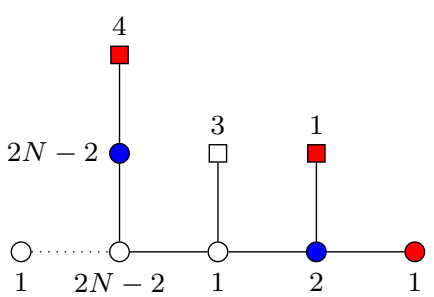

(b) Orthosymplectic quiver

Figure 28. Magnetic quivers for the $\hat{K}_{N}^{1}$ theory

\begin{tabular}{|c|c|c|}
\hline \multirow{2}{*}{$\hat{K}_{N}^{1}$} & Unitary magnetic quiver & Orthosymplectic magnetic quiver \\
\cline { 2 - 3 } & $\mathrm{HS}_{\mathbb{H}}(t)$ & $\mathrm{HS}_{\mathbb{H}}(t)$ \\
\hline$\hat{K}_{2}^{1}$ & $1+9 t^{2}+6 t^{3}+36 t^{4}+36 t^{5}+112 t^{6}+120 t^{7}+$ & $1+9 t^{2}+6 t^{3}+36 t^{4}+36 t^{5}+112 t^{6}+120 t^{7}+$ \\
$2855 t^{8}+\ldots$ \\
\hline$\hat{K}_{3}^{1}$ & $1+16 t^{2}+6 t^{3}+150 t^{4}+86 t^{5}+981 t^{6}+\ldots$ & $1+16 t^{2}+6 t^{3}+150 t^{4}+86 t^{5}+981 t^{6}+\ldots$ \\
\hline
\end{tabular}

Table 11. Higgs branch Hilbert series of the unitary and orthosymplectic magnetic quivers presented in figure 28.

In our case, bare SI with half D5-brane is one. The contribution from the half D7-brane is also one because the considered subweb and the half D5 are both attached to the common half D7-brane from the same direction. ${ }^{7}$ We propose that the number of charge 1 hypermultiplets coupled to the $\mathrm{U}(1)$ node is given by (3.6) in general. The contribution from the common half D7-brane on the $\mathrm{O}^{+}$-plane is computed analogously to the case for the unitary quiver discussed in [45].

To support this proposed rule, we match the Higgs branch Hilbert series for the unitary and the orthosymplectic magnetic quiver, especially to settle the question of whether the $\mathbb{Z}_{2}$ node is gauge or flavor. We find that only choosing the $\mathbb{Z}_{2}$ node to be gauge we recover the correct match. We computed the Hilbert series for both $N=2$ and $N=3$, and the result is tabulated in table 11 .

\subsection{The $X_{N}^{1,1}$ theory}

We then consider the configuration obtained by intersecting $2 N$ NS5s, one $(1,1)$, and one $(1,-1)$ on top of an $\mathrm{O} 5^{+}$-plane (figure 29$)$. We call the theory on the web the $X_{N}^{1,1}$ theory. There is a corresponding IR gauge theory description as

$$
\overbrace{\mathrm{SO}(6)-\mathrm{USp}(2)-\mathrm{SO}(6)-\cdots-\mathrm{USp}(2)-\mathrm{SO}(6)}^{2 N+1}
$$

\footnotetext{
${ }^{7}$ When we compute the contribution from the common half D7-brane on the $\mathrm{O} 5^{+}$-plane, we treat it as if there were only a single half D5-brane terminated at the common half D7-brane. We do not multiply 4 for this contribution contrary to the contribution from SI.
} 




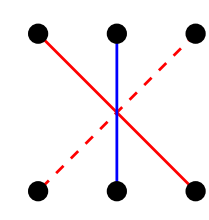

(I)



(II)

Figure 29. An orientifold web for the $X_{N}^{1,1}$ theory with asymptotically $\mathrm{O} 5^{+}$planes. We show the two possible maximal subdivisions on the right.

$(3,1)$
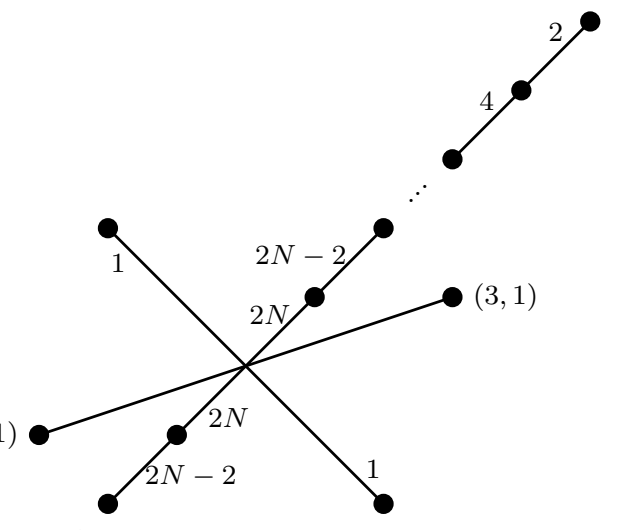

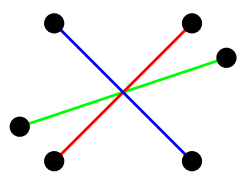

(I)



(II)

Figure 30. A unitary web for the $X_{N}^{1,1}$ theory with asymptotically $\mathrm{O} 5^{+}$orientifold planes. The two possible maximal subdivisions are shown on the right.

Alternatively, it may be understood as gluing $N-1$ copies of $\mathrm{SO}(6)$ with 2 vectors and two copies of $\mathrm{SO}(6)$ with one vector, via successive gauging of $\operatorname{USp}(2)$ subgroups of the flavour symmetry. This allows us to construct a unitary web for this theory by gluing $N-1$ copies of SU(4) $)_{0}$ with 2 antisymmetric hypermultiplets and two copies of $\mathrm{SU}(4)_{0}$ with one antisymmetric hypermultiplet, via successive gauging of $\mathrm{SU}(2)$ subgroups of the global symmetry (figure 30).

The magnetic quivers for the $X_{N}^{1,1}$ theory are given in table 12 and the Coulomb branch Hilbert series are tabulated in table 13 . 


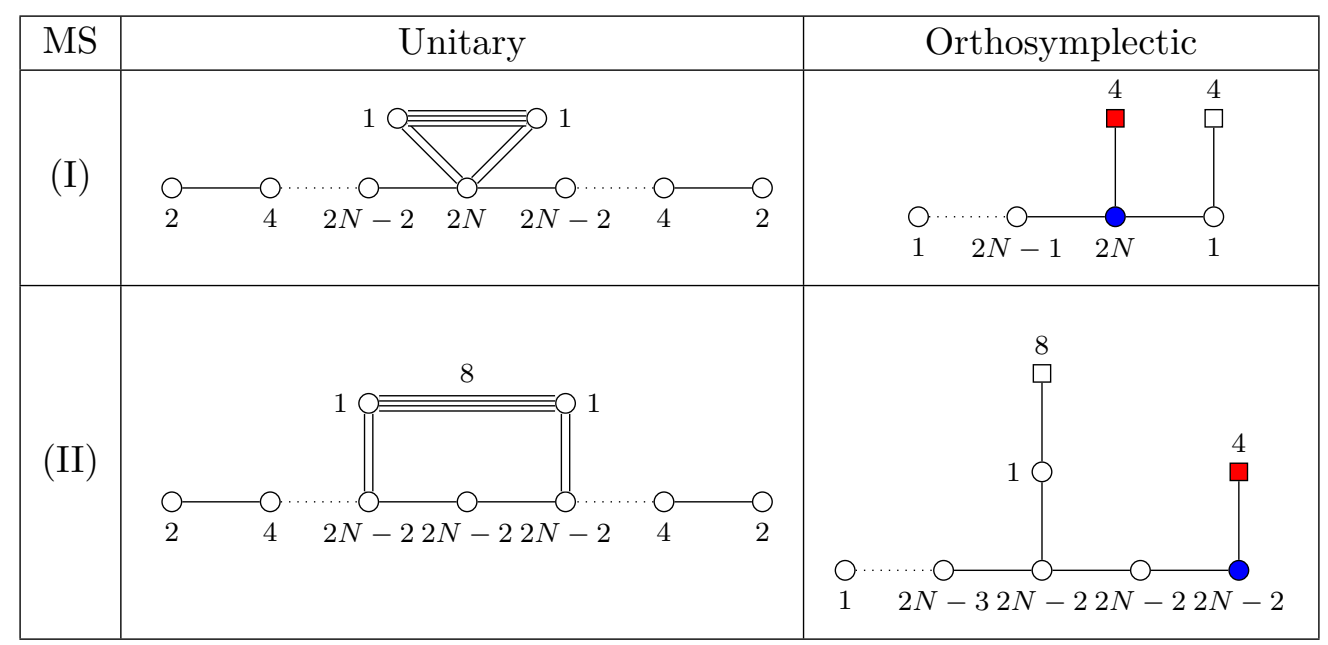

Table 12. Magnetic quivers corresponding to the maximal subdivisions for the $X_{N}^{1,1}$ theory.

\begin{tabular}{|c|c|c|c|}
\hline \multirow{2}{*}{ MS } & Unitary magnetic quiver & \multicolumn{2}{|c|}{ Orthosymplectic magnetic quiver } \\
\cline { 2 - 4 } & $\operatorname{HS}(t)$ & $\operatorname{HS}(t ; \vec{m} \in \mathbb{Z})$ & HS $\left(t ; \vec{m} \in \mathbb{Z}+\frac{1}{2}\right)$ \\
\hline \multirow{3}{*}{$(\mathrm{I})_{N=1}$} & $\frac{P_{19}(t)}{(1-t)^{2}\left(1-t^{3}\right)\left(1-t^{4}\right)^{3}}$ & $\frac{P_{19}(t)}{(1-t)^{2}\left(1-t^{3}\right)\left(1-t^{4}\right)^{3}}$ & not required \\
& $=1+4 t+13 t^{2}+33 t^{3}$ & $=1+4 t+13 t^{2}+33 t^{3}$ & \\
& $+80 t^{4}+165 t^{5}+\cdots$ & $+80 t^{4}+165 t^{5}+\cdots$ & not required \\
\hline$(\mathrm{I})_{N=2}$ & $1+16 t+151 t^{2}+1039 t^{3}+$ & $1+16 t+151 t^{2}+1039 t^{3}+$ & not required \\
\hline \multirow{2}{*}{$(\mathrm{II})_{N=2}$} & $5750 t^{4}+26954 t^{5}+\cdots$ & $5750 t^{4}+26954 t^{5}+\cdots$ & not required \\
\hline$(\mathrm{II})_{N=3}$ & $1+16 t+151 t^{2}+1004 t^{3}+$ & $1+16 t+151 t^{2}+1004 t^{3}+$ & $5198 t^{4}+22184 t^{5}+\cdots$ \\
\hline
\end{tabular}

Table 13. Coulomb branch Hilbert series for the unitary and orthosymplectic magnetic quivers presented in table 12. The explicit form of $P_{19}(t)$ is provided in appendix B.

\section{Magnetic quivers from $\mathrm{O5}^{-}-\mathrm{O5}^{+}$}

So far we have focused on the configurations where the two asymptotic orientifold planes are of the same type. It is possible to consider cases with an $\mathrm{O}^{-}$-plane on one end and an $\mathrm{O} 5^{+}$-plane on the other end. We will consider such examples in this section.

\subsection{The ${ }_{+}^{3,1}$ theory}

For obtaining the configuration which has an $\mathrm{O} 5^{-}$-plane on one end and an $\mathrm{O} 5^{+}$-plane on the other end, we decouple one $\operatorname{USp}(2)$ part from the quiver in (2.1) for $M=3$. An IR quiver description of the theory is

$$
[1]-\mathrm{SO} \overbrace{(6)-\mathrm{USp}(2)-\mathrm{SO}(6)-\cdots-\mathrm{USp}(2)-\mathrm{SO}(6)-\mathrm{USp}(2)}^{2 N-2}-[3] .
$$

The orientifold web for the $+{ }_{N}^{3,1}$ theory is presented in figure 31 . 



Figure 31. Orientifold web diagram for the $+_{N}^{3,1}$ theory.

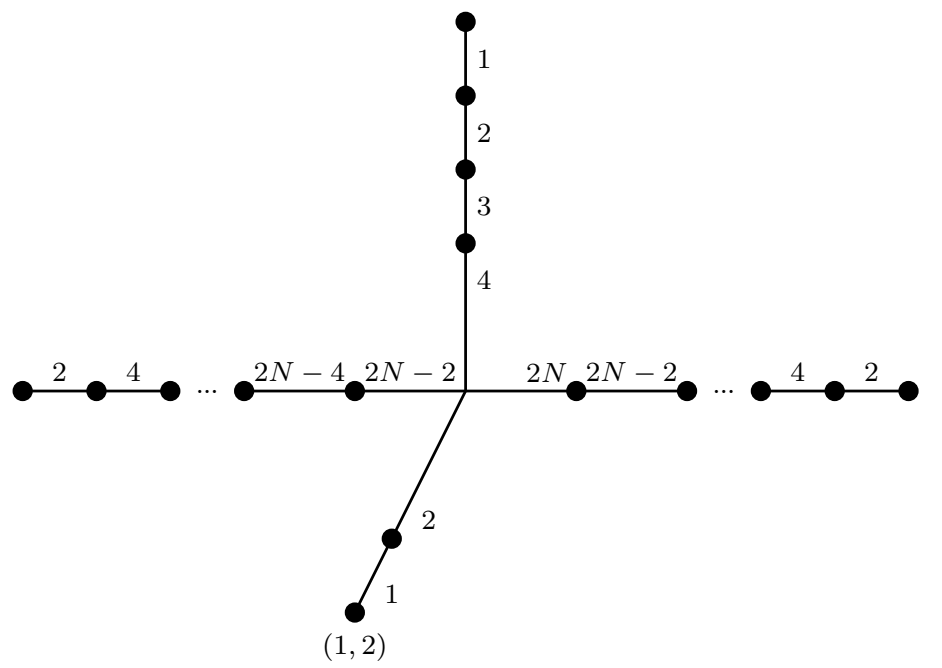

Figure 32. Unitary web diagram for $+{ }_{N}^{3,1}$ theory.

The corresponding unitary web is depicted in figure 32, which is obtained as follows: We first interpret the $+_{N}^{3,1}$ theory as a decoupling limit from the $\#_{3, N}$ theory. The discussion will be clearer when we discuss this decoupling limit in the S-dual description. The S-dual description of the $\#_{3, N}$ theory has a low energy description as a $5 \mathrm{~d} D_{3}$ quiver gauge theory as given in (2.3). The corresponding 5 -brane web for this $5 \mathrm{~d} D_{3}$ quiver gauge theory with $N=2$ is depicted in figure 33, which is related to the web diagram in figure 1 by S-duality as well as "generalized flop transition" discussed in [22]. The decoupling limit is to move the red D5-branes downward while keeping the other D5-branes' positions intact, as in figure 33. These D5-branes are the lowest color branes for each gauge node and the lowest flavor brane charged under the central $\mathrm{SU}(2 N)$. By this decoupling limit, we obtain the 5-brane web in figure 34, which is the S-dual description of the $+{ }_{N}^{3,1}$ theory. Since $D_{3}=A_{3}$, we can consider the corresponding decoupling process also in the ordinary 5-brane web. The ordinary 5 -brane web for the $A_{3}$ quiver gauge theory is depicted in figure 35 . The corresponding decoupling limit is again to move the red D5-branes downward while keeping 


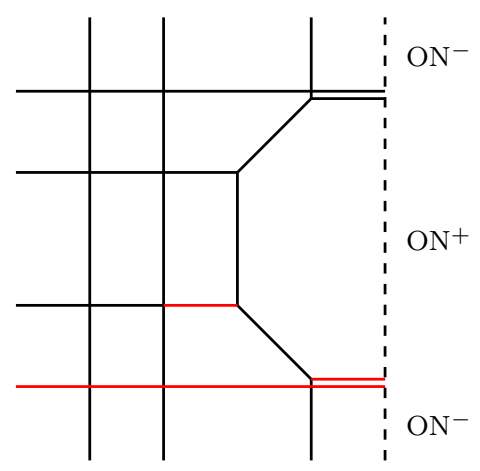

Figure 33. S-dual of $\#_{3,2}$ theory. We move four red D5-branes downward for decoupling.

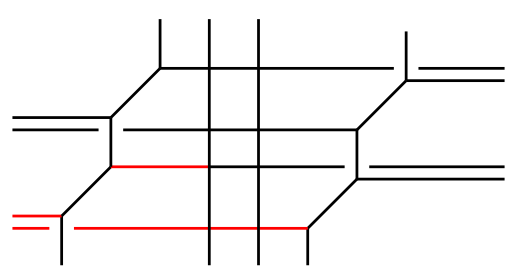

Figure 35. Usual 5-brane web for $A_{3}$ quiver for $N=2$. We move four red D5-branes downward for decoupling.

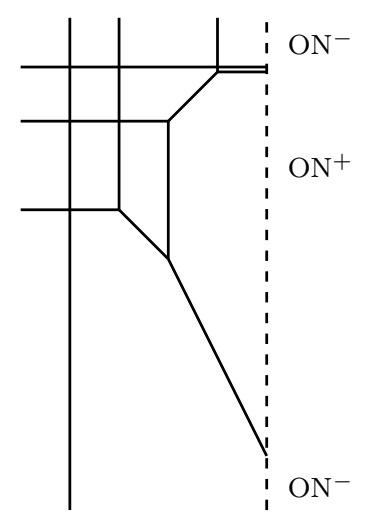

Figure 34. 5-brane web for $+{ }_{N}^{3,1}$ theory obtained from $\#_{3,2}$ theory by the decoupling limit.

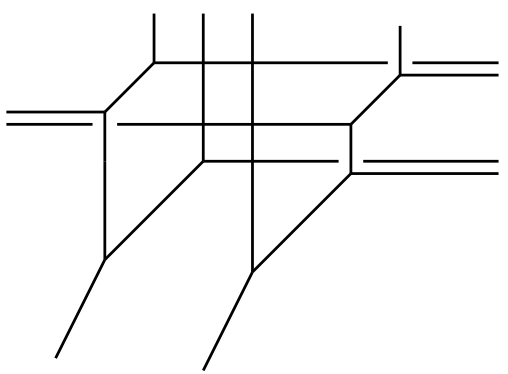

Figure 36. 5-brane web obtained from the $A_{3}$ quiver by the decoupling.

the other D5-branes' positions intact, as in figure 35. Then, we obtain a 5-brane web depicted in figure 36. Since it is obtained from the same decoupling limit from the $\#_{3, N}$ theory, this should correspond to the $+{ }_{N}^{3,1}$ theory. The strong coupling limit of figure 36 gives figure 32 after S-duality. Although this explanation is for the case with $N=2$, generalization for generic $N$ is straightforward.

The main part of the maximal subdivision of the orientifold web is given in figure 31 . One of the new features in this example compared to the ones in the previous sections is the dotted blue line on the $\mathrm{O}^{+}$-plane connected to the ordinary blue line on the $05^{-}$-plane. The dotted blue line represents one of the four half D5-branes included in the $05^{+}$-plane, which is based on the interpretation discussed around (3.6), while the ordinary blue line is the half D5-brane coming from the three full D5-branes on the $\mathrm{O}^{-}{ }^{-}$-plane. Together with the other two ordinary blue half D5-branes, it contributes as a $\mathrm{SO}(3)$ gauge node in the OSp magnetic quiver in figure 37. Since three half D5-branes are consumed to construct the $\mathrm{SO}(3)$ gauge node, three red half $\mathrm{D} 5$-branes remain on the $\mathrm{O} 5^{-}$-plane. Due to the charge conservation and the s-rule, the red part, as well as O5-plane, should be treated as one subweb, where the number of half D5 branes reduce by one as we go over half D7-branes to the right. Due to this red subweb, the $\mathrm{SO}(3)$ gauge node appears from the part where there are originally two full D5-branes on the $\mathrm{O} 5^{-}$-plane because a single half 
D5-brane is used as a part of the red subweb, and only three half D5-branes remain in this region. This red subweb cannot be detached from the O5-plane because there is no mirror pair and thus contributes as fundamental hypermultiplet to various gauge nodes. The number of half hypermultiplets is obtained by computing the stable intersection number between the subweb corresponding to the considered gauge node and this red subweb, which includes both the original and its mirror image. For example, the stable intersection number between the red subweb and the green NS5-brane is three. However, they both attach to the same $(0,1) 7$-branes from the same direction at the two places, including the mirror image so that the number of the half-hypermultiplets for the $\operatorname{USp}(2 N-2)$ gauge node is $3-2=1$. Or, equivalently, we could have considered that the green NS5-branes are placed to the left of the red subweb so that it does not intersect with it. In this case, we should regard that the green NS5-branes are intersecting with $\mathrm{O} 5^{+}$-plane. Then, the rule discussed below (3.6) enables us to reinterpret the stable intersection number with the red subweb as a stable intersection number with the 3 half D5-branes inside $05^{+}$-plane, whichever interpretation gives a consistent magnetic quiver.

Some part of the structure in the magnetic quiver discussed above may be more natural to understand if we consider Hanany-Witten transition. Suppose we concentrate only on the red subweb as well as remaining D5-branes on the O5-planes while omitting the remaining part. By moving three half D7-branes from the right to the left of the red subweb, we obtain a simpler web diagram, from which we can straightforwardly read off most of the $\mathrm{SO}$ and the USp gauge nodes in the magnetic quiver. This discussion is parallel to the 5 -brane analysis for the Higgs branch of the $5 \mathrm{~d} \mathrm{Sp}(\mathrm{N})$ gauge theory at finite coupling in [1]. This observation would be useful to partially support our magnetic quiver but if we need to obtain the full structure of the magnetic quiver, the original orientifold web would be more convenient.

Finally, to understand if the $\mathbb{Z}_{2}$ node in the magnetic quiver of figure 37 is gauge or flavor, we computed the Hilbert series of the Higgs branch in both cases, and compared with the unitary magnetic quiver. We find that only when we take $\mathbb{Z}_{2}$ node to be gauge, the match is recovered. The Higgs branch Hilbert series reads

$$
\mathrm{HS}_{H}(t)=1+t^{2}+2 t^{3}+5 t^{4}+6 t^{5}+O\left(t^{6}\right)
$$




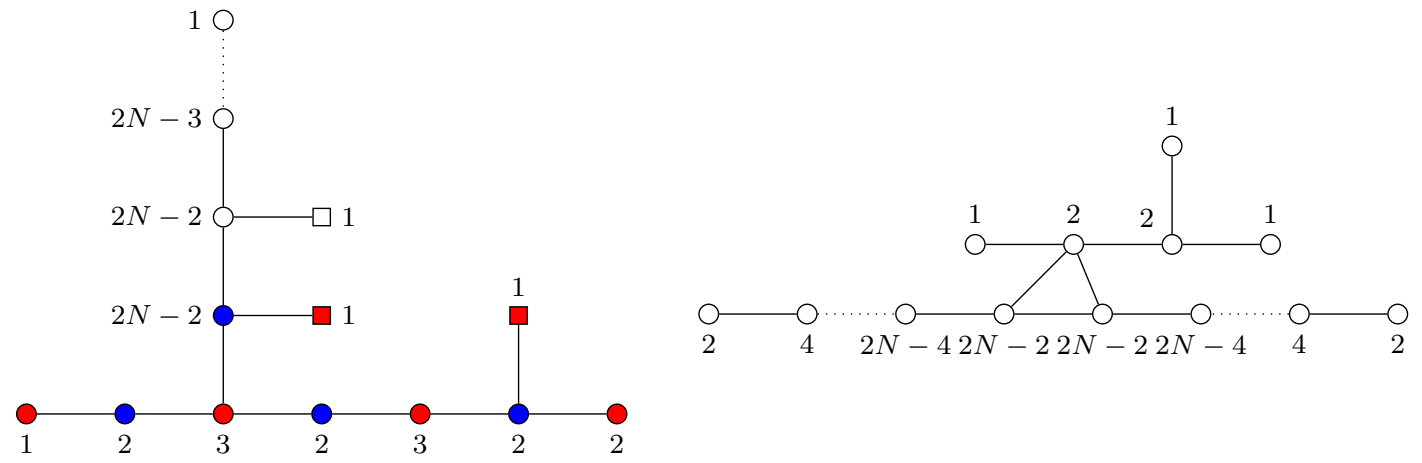

Figure 37. Magnetic quivers for the $+{ }_{N}^{3,1}$ theory.

\subsection{The $\tilde{K}_{N}^{1}$ theory}

The $\tilde{K}_{N}^{1}$ theory in the class of $\mathrm{O} 5^{-}-\mathrm{O} 5^{+}$is obtained from the $+_{N+1}^{3,1}$ theory by decoupling a flavor from (4.1). At low energies, there is a gauge theory description

$$
\mathrm{SO}(\overbrace{6)-\mathrm{USp}(2)-\mathrm{SO}(6)-\cdots-\mathrm{USp}(2)-\mathrm{SO}(6)-\mathrm{USp}}^{2 N}(2)-[3]
$$

The orientifold web for the $\tilde{K}_{N}^{1}$ theory is presented in figure 38 .

In figure 34 and figure 36 , the $\mathrm{SU}(2 N+1)$ gauge node has $2 N+1$ flavor, and is coupled to a non-Lagrangian theory, where the figures are depicted for the case $N=1$. Decoupling one flavor from the $\mathrm{SU}(2 N+1)$ gauge node, we obtain the web diagrams in figure 39 .

The $\tilde{K}_{N}^{1}$ theory has two maximal subdivisions, as in figure 38 . In maximal subdivision (I), the red subweb cannot be detached from the O5-plane and thus, giving fundamental hypermultiplets to the $\operatorname{USp}(2 N)$ node coming from the $2 N$ NS5-branes intersecting with the O5-planes. In maximal subdivision (II), there is a blue dotted line on the $\mathrm{O} 5^{+}$-plane connected to the ordinary blue line on the $\mathrm{O}^{-}$-plane, analogous to the case for the $+_{N}^{3,1}$ theory. However, this does not become part of a gauge node because there are no other half D5-branes available at the same place. Therefore, both the blue subweb and the red subweb contribute as fundamental hypermultiplets of various gauge nodes. The magnetic quivers are given in table 14 .

To understand if the two $\mathbb{Z}_{2}$ nodes in the magnetic quiver associated with the maximal subdivision (II) are gauge or flavor, we computed the Hilbert series of the Higgs branch in both cases and compared with the unitary magnetic quiver. We find that only when we take both $\mathbb{Z}_{2}$ nodes to be flavor, the match is recovered. The Higgs branch Hilbert series reads

$$
\mathrm{HS}_{H}(t)=1+5 t^{2}+8 t^{3}+18 t^{4}+36 t^{5}+71 t^{6}+120 t^{7}+\cdots .
$$

The Coulomb branch Hilbert series is tabulated in table 15. 

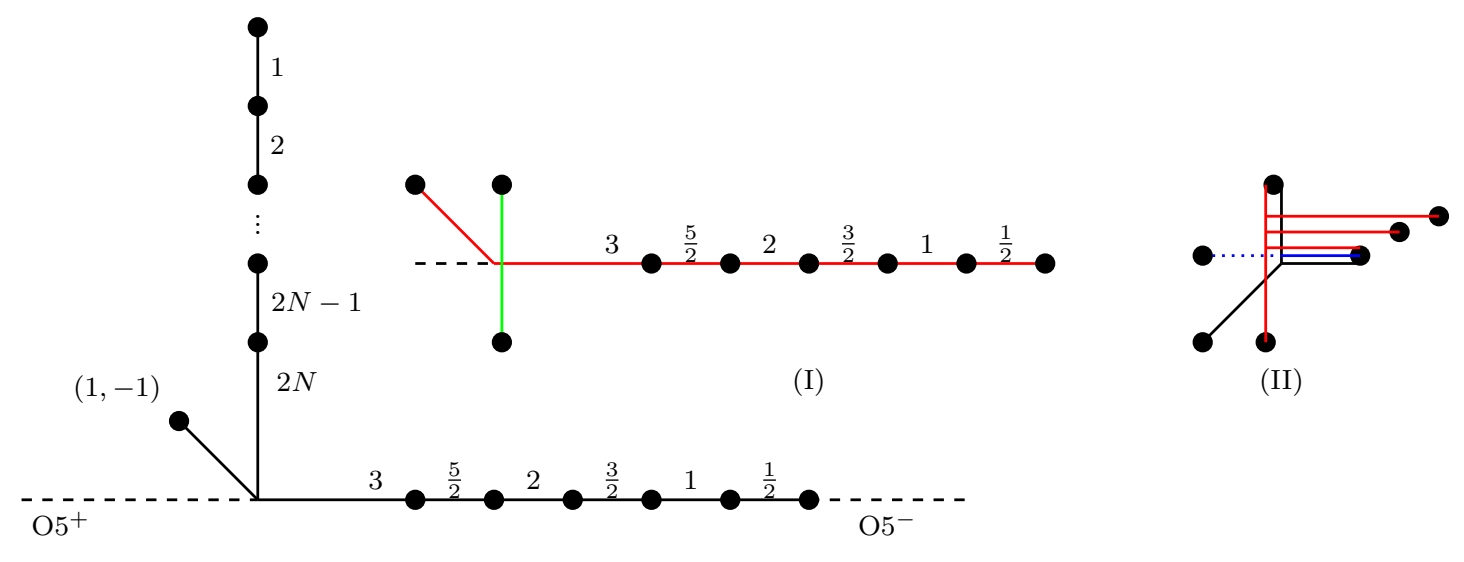

(II)

Figure 38. An orientifold web for the $\tilde{K}_{N}^{1}$ theory.

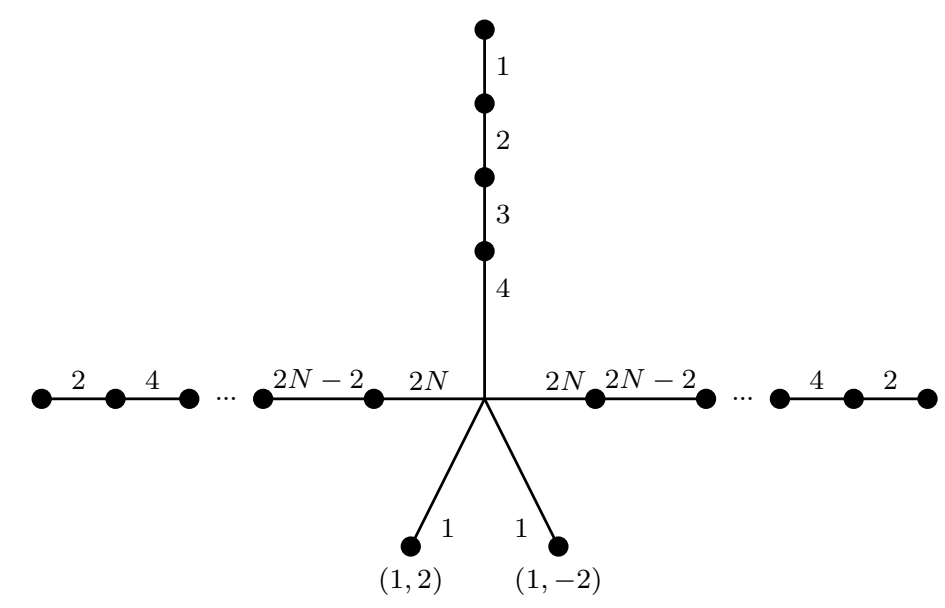

Figure 39. A unitary web description of the $\tilde{K}_{N}^{1}$ theory.



Table 14. Magnetic quivers for various maximal subdivisions (MS) of the $\tilde{K}_{N}^{1}$ theory. 


\begin{tabular}{|c|c|c|c|}
\hline \multirow{2}{*}{ MS } & Unitary magnetic quiver & \multicolumn{2}{|c|}{ Orthosymplectic magnetic quiver } \\
\cline { 2 - 4 } & $\operatorname{HS}(t)$ & $\operatorname{HS}(t ; \vec{m} \in \mathbb{Z})$ & $\operatorname{HS}\left(t ; \vec{m} \in \mathbb{Z}+\frac{1}{2}\right)$ \\
\hline \multirow{3}{*}{$(\mathrm{I})_{N=1}$} & $\frac{1+t+2 t^{2}+t^{3}+t^{4}}{(1-t)^{4}(1+t)^{2}}$ & $\frac{1+t+2 t^{2}+t^{3}+t^{4}}{(1-t)^{4}(1+t)^{2}}$ & not required \\
& $=1+3 t+9 t^{2}+18 t^{3}$ & $=1+3 t+9 t^{2}+18 t^{3}$ & \\
& $+35 t^{4}+57 t^{5}+\cdots$ & $+35 t^{4}+57 t^{5}+\cdots$ & not required \\
\hline$(\mathrm{I})_{N=2}$ & $1+15 t+135 t^{2}+888 t^{3}+4709 t^{4}+$ & $1+15 t+135 t^{2}+888 t^{3}+4709 t^{4}+$ & not required \\
\hline & $21144 t^{5}+\cdots$ & $21144 t^{5}+\cdots$ & \\
$(\mathrm{II})_{N=1}$ & $1+19 t+173 t^{2}+24 t^{5 / 2}+$ & $1+19 t+173 t^{2}+24 t^{5 / 2}+$ & not required \\
& $1042 t^{3}+328 t^{7 / 2}+4760 t^{4}+$ & $1042 t^{3}+328 t^{7 / 2}+4760 t^{4}+$ & $2312 t^{9 / 2}+17908 t^{5}+\cdots$ \\
\hline
\end{tabular}

Table 15. Coulomb branch Hilbert series for the unitary and orthosymplectic magnetic quivers presented in table 14 for the $\tilde{K}_{N}^{1}$ theory. 


\section{Magnetic quivers from $\widetilde{\mathrm{O5}}^{+}$}

Finally we consider some examples where we have an $\widetilde{\mathrm{O} 5}^{+}$-plane on one or two of the ends in the orientifold web configuration.

\section{$5.1 \widetilde{\mathrm{O} 5}^{+}-\widetilde{\mathrm{O} 5}^{+}$}

We begin with an example where we have $\widetilde{\mathrm{O} 5}^{+}$-planes on the two ends of the brane configuration. For constructing such a configuration we start from the theory considered in section 3.1, which yields the orthosymplectic quiver (3.1) as an IR theory. From the quiver theory (3.1), we Higgs the two $\mathrm{SO}(6)$ gauge theories on the ends. The resulting IR theory becomes

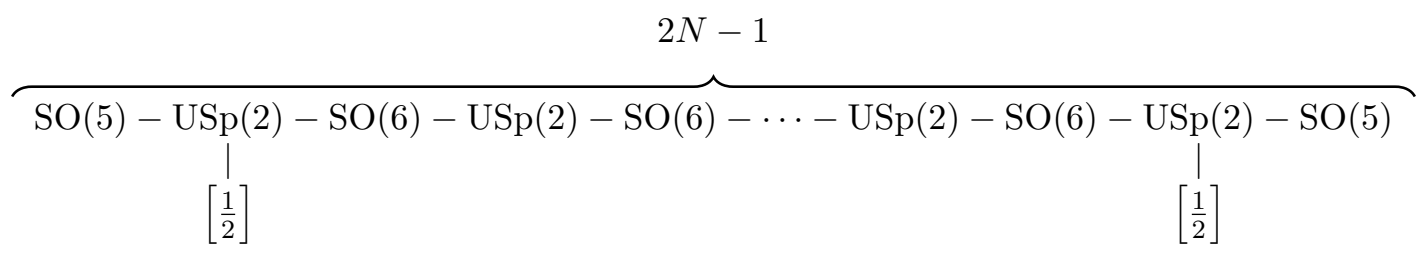

where $\frac{1}{2}$ represents a half-hypermultiplet in the fundamental representation of USp(2). It is possible to construct the corresponding orientifold web diagram by performing the same Higgsing to the web in figure 23. The orientifold web diagram is depicted in figure 40 which has asymptotically $\widetilde{\mathrm{O} 5}^{+}$-planes. From the orientifold web configuration the presence of a half D7-brane accounts for the half-hypermultiplet.

The theory in (5.1) can also be constructed by gluing 2 copies of $\mathrm{SO}(5)$ with 1 vector and 1 singlet, and $N-2$ copies of $\mathrm{SO}(6)$ with two vectors via successive gauging of USp(2) subgroup of the global symmetry. This allows us to propose a unitary web in figure 40 by gluing together 2 copies of USp(4) with one antisymmetric hypermultiplet and one singlet, and $N-2$ copies of $\mathrm{SU}(4)_{0}$ with two antisymmetric hypermultiplets via gauging $\mathrm{SU}(2)$ subgroups of the global symmetry.

The orthosymplectic and unitary magnetic quiver theories can be read off from the web diagrams in figure 40, and they are given in figure 41. The corresponding Coulomb branch Hilbert series are given in table 16. 


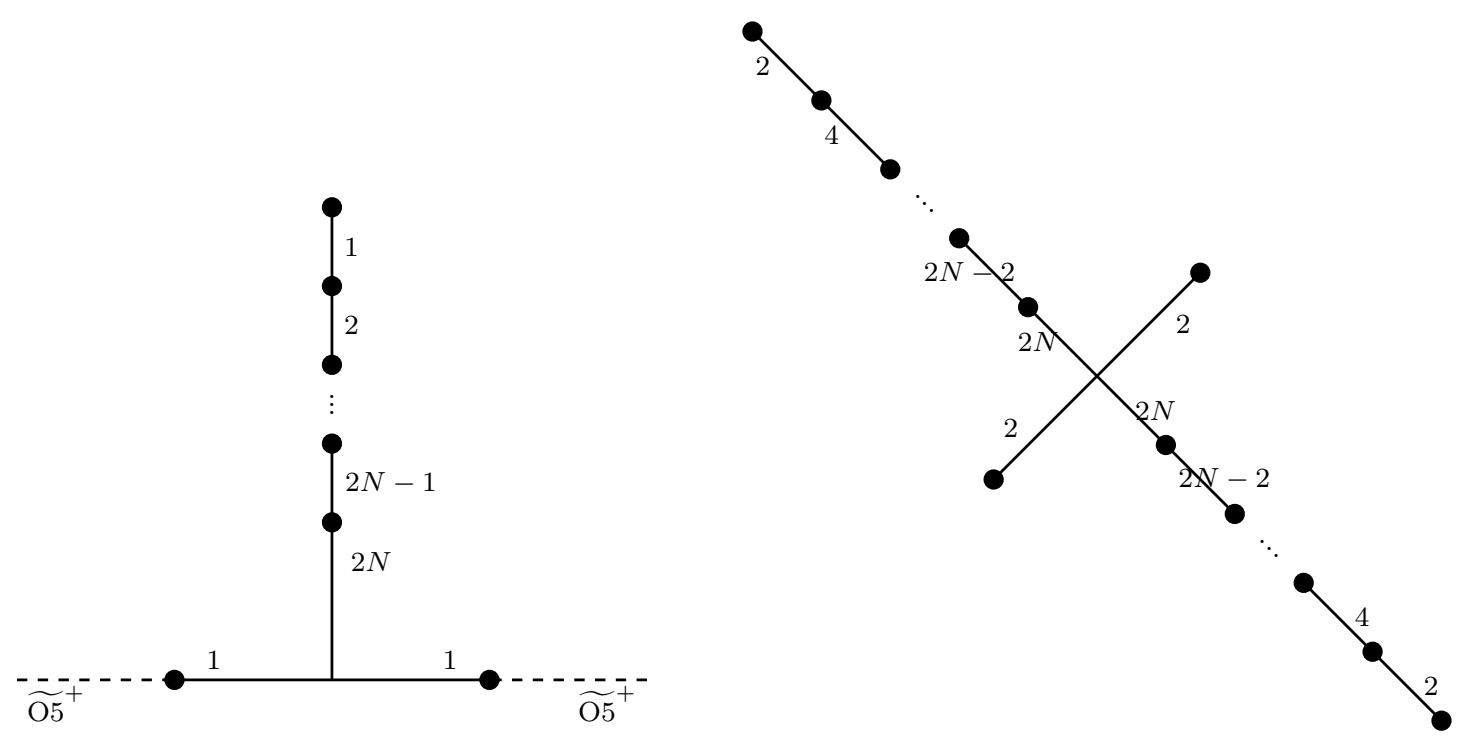

Figure 40. Orientifold web(left), and ordinary web(right) for the $+_{N}$ theory. The maximal subdivision is the trivial one and therefore not shown explicitly.

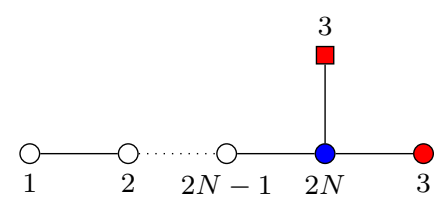

(a) Orthosymplectic quiver

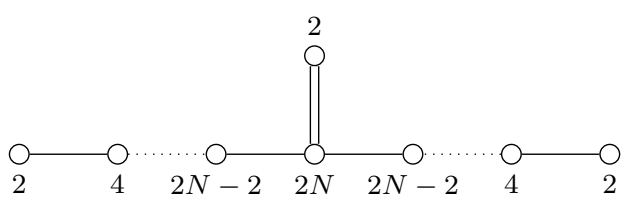

(b) Unitary quiver

Figure 41. Magnetic quivers for the $+_{N}$ theory

\begin{tabular}{|c|c|c|c|}
\hline \multirow{2}{*}{$+_{N}$} & Unitary magnetic quiver & Orthosymplectic magnetic quiver \\
\cline { 2 - 4 } & $\operatorname{HS}(t)$ & $\operatorname{HS}(t ; \vec{m} \in \mathbb{Z})$ & HS $\left(t ; \vec{m} \in \mathbb{Z}+\frac{1}{2}\right)$ \\
\hline$+_{1}$ & bad theory & bad theory & not required \\
\hline \multirow{2}{*}{$+_{2}$} & $1+16 t+168 t^{2}+1315 t^{3}+$ & $1+16 t+168 t^{2}+1315 t^{3}+$ & not required \\
\hline
\end{tabular}

Table 16. Coulomb branch Hilbert series for the unitary and orthosymplectic magnetic quivers presented in figure 41 for the $+_{N}$ theory.

\section{$5.2 \widetilde{\mathrm{O} 5}^{+}-\mathrm{O5}^{+}$}

It is also possible to consider an example where we have an $\widetilde{\mathrm{O} 5}^{+}$-plane on one end and an $\mathrm{O} 5^{+}$-plane on the other end. We again start from the theory considered in section 3.1, which yields the orthosymplectic quiver (3.1) as an IR theory. From the quiver theory (3.1), we Higgs the $\mathrm{SO}(6)$ gauge theory on the right end and decouple flavor from the $\mathrm{SO}(6)$ gauge 

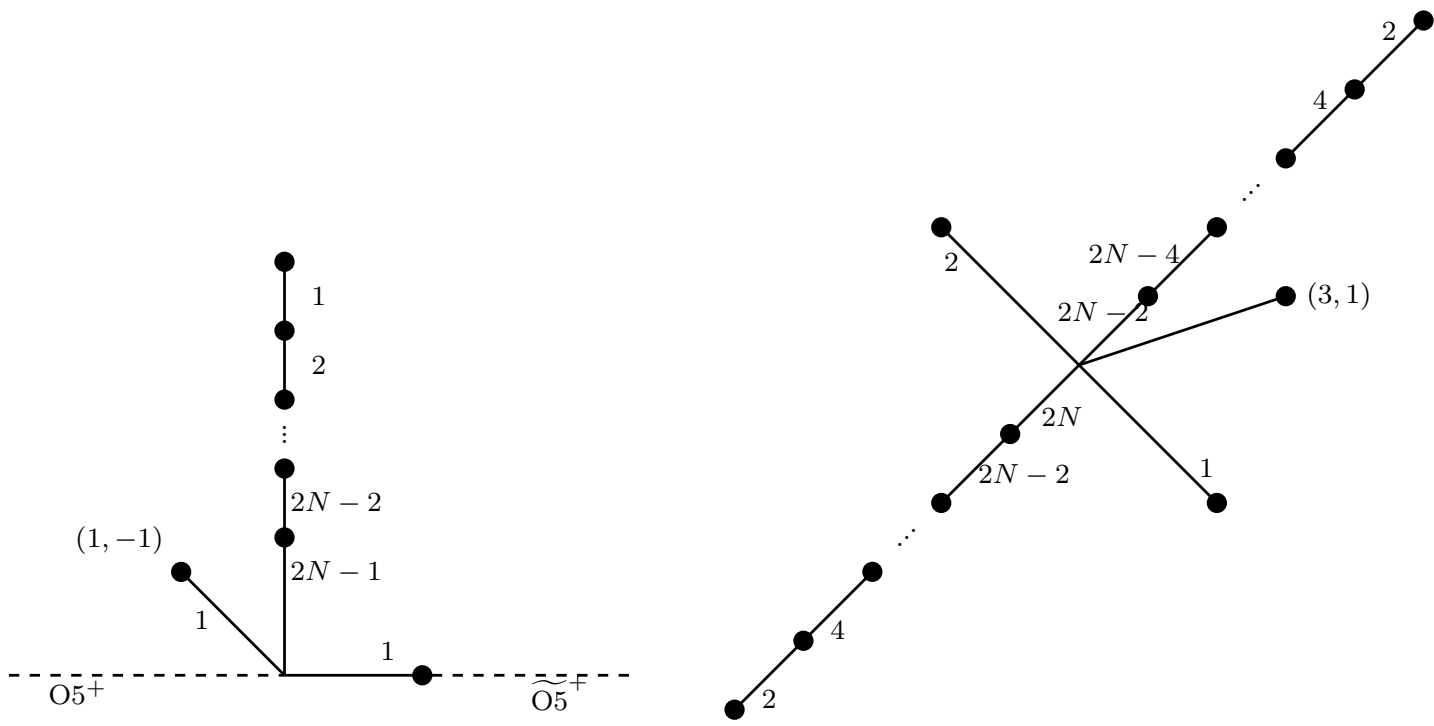

Figure 42. Orientifold and unitary web diagram for the $\kappa_{N}^{1}$ theory.

on the left end. Then the resulting IR theory becomes

$$
[1]-\overbrace{\mathrm{SO}(6)-\mathrm{USp}(2)-\mathrm{SO}(6)-\cdots-\mathrm{SO}(6)-\mathrm{USp}(2)-\mathrm{SO}(5)}^{2 N-1}
$$

We can construct the corresponding orientifold web diagram by performing the same Higgsing and the decoupling to the web in figure 23. The orientifold web diagram is depicted in figure 42 which has asymptotically an $\widetilde{\mathrm{O} 5}^{+}$-plane and an $\mathrm{O} 5^{+}$-plane.

This theory in (5.2) can also be made by gluing 1 copy of $\mathrm{SO}(5)$ with 1 vector and 1 singlet, and $N-1$ copies of $\mathrm{SO}(6)$ with two vectors via successive gauging of $\operatorname{USp}(2)$ subgroup of the global symmetry. This allows us to propose the corresponding unitary web in figure 42 by gluing together 1 copy of $\mathrm{USp}(4)$ with one antisymmetric hypermultiplet and one singlet, and $N-1$ copies of $\mathrm{SU}(4)_{0}$ with two antisymmetric hypermultiplets via gauging $\mathrm{SU}(2)$ subgroups of the global symmetry.

The orthosymplectic and unitary magnetic quiver theories can be read off from the web diagrams in figure 42, and they are given in figure 43. The corresponding Coulomb branch Hilbert series is presented in table 17 . 


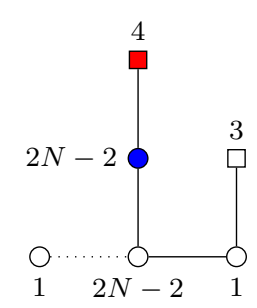

(a) Orthosymplectic quiver



(b) Unitary quiver

Figure 43. Magnetic quivers for the $\kappa_{N}^{1}$ theory

\begin{tabular}{|c|c|c|c|}
\hline \multirow{2}{*}{$K_{N}^{1}$} & Unitary magnetic quiver & \multicolumn{2}{|c|}{ Orthosymplectic magnetic quiver } \\
\cline { 2 - 4 } & $\operatorname{HS}(t)$ & $\operatorname{HS}(t ; \vec{m} \in \mathbb{Z})$ & $\operatorname{HS}\left(t ; \vec{m} \in \mathbb{Z}+\frac{1}{2}\right)$ \\
\hline \multirow{2}{*}{$K_{2}^{1}$} & $1+9 t+53 t^{2}+6 t^{5 / 2}+227 t^{3}+$ & $1+9 t+53 t^{2}+6 t^{5 / 2}+227 t^{3}+$ & not required \\
\hline$K_{3}^{1}$ & $1+25 t+349 t^{2}+3499 t^{3}+\cdots$ & $1+25 t+349 t^{2}+3499 t^{3}+\cdots$ & not required \\
\hline
\end{tabular}

Table 17. Coulomb branch Hilbert series for the unitary and orthosymplectic magnetic quivers presented in figure 43 for the $\kappa_{N}^{1}$ theory.

\section{Conclusion}

In this paper, we studied infinite coupling Higgs branches of 5d superconformal theories based on 5-brane webs, by constructing $3 \mathrm{~d}$ magnetic quivers whose Coulomb branch yields the Higgs branch of the $5 \mathrm{~d}$ system. Our primary focus was $5 \mathrm{~d}$ theories, which can be engineered by 5 -brane webs with $\mathrm{O} 5$-planes and also without O5-planes by either S-duality or some gauging subalgebra of flavor symmetry. As a 5-brane web without an O5-plane gives a unitary magnetic quiver, while that with an O5-plane gives an orthosymplectic magnetic quiver, these $5 \mathrm{~d}$ theories of two different 5-brane web descriptions should yield the same Higgs branch. In other words, the Coulomb branches from the corresponding unitary and orthosymplectic magnetic quivers should agree. We employed this fact to further develop how to construct orthosymplectic magnetic quivers [1] by comparing the counterpart unitary magnetic quiver, in particular, by explicitly checking the Hilbert series for both magnetic quivers. With various decoupling limits for both 5-brane webs with/without O5-planes, we proposed a generalization of the rules for constructing $3 \mathrm{~d}$ magnetic quivers from 5-brane webs with O5-planes. The novel features that we found include (i) generalized stable intersection number involving subwebs intersecting with its mirror through an O5-plane as well as the stable intersection with an O5-plane, which in turn determines the number of charge 2 matter appearing in the magnetic quivers, (ii) a new type of hypermultiplet transforming in the fundamental-fundamental representation of two gauge nodes, (iii) appearance of matter in the antisymmetric representation of gauge nodes. (iv) possibility of decomposable and not-decomposable for seeming equivalent subwebs depending the discrete theta angles for 5-brane configurations for 5 d USp gauge groups, and (v) $\mathbb{Z}_{2}$ gauge nodes. 
We also checked the matching of both the Coulomb branch and the Higgs branch Hilbert series for all the unitary and orthosymplectic magnetic quivers appearing in this work (though we provided the Higgs branch Hilbert series only when it was required). Having checked that each pair of unitary and orthosymplectic magnetic quivers have isomorphic Coulomb and Higgs branch moduli space of vacua, it is natural to suggest a possible duality between each pair. It would be interesting to check this duality more systematically, for instance, via superconformal indices or partition functions. In fact, we checked the partition functions for some $3 \mathrm{~d}$ theories and found that their partition functions can be mapped to each other by a simple fugacity map. Understanding the $4 \mathrm{~d}$ origin of this duality, should it exist, would be another exciting direction to pursue.

Though our construction applies to generic $5 \mathrm{~d}$ theories of any rank, some lower rank theories possess special dualities [39]. In particular, 5-brane webs for most of rank 2 superconformal theories have been constructed [23], a systematic study of their magnetic quivers would also shed some light on a better understanding of orthosymplectic magnetic quivers [54]. Some magnetic quivers of the rank 2 theories were also considered in $[51,55,56]$.

\section{Acknowledgments}

We thank Julius Grimminger, Kimyeong Lee, and Dominik Miketa for useful discussions. We would like to especially thank Amihay Hanany for suggesting half-integer sum of orthosymplectic magnetic quivers and also useful discussions. SSK thanks the hospitality of KIAS, POSTECH, and Sichuan University, where part of work is done, and also APCTP for hosting the Focus program "Strings, Branes and Gauge Theories 2020." MA is grateful for the hospitality of Sichuan University during the earlier stages of this project. MA is supported by an STFC grant ST/S505778/1. The work of HH is supported in part by JSPS KAKENHI Grant Number JP18K13543. FY is supported by the NSFC grant No. 11950410490, by Fundamental Research Funds for the Central Universities A0920502051904-48, by Start-up research grant A1920502051907-2-046, in part by NSFC grant No. 11501470 and No. 11671328, and by Recruiting Foreign Experts Program No. T2018050 granted by SAFEA. FC is supported by the ERC Consolidator Grant STRINGFLATION under the HORIZON 2020 grant agreement no. 647995.

\section{A Hilbert series computations}

\section{A.1 Coulomb branch Hilbert series}

Here we briefly review the computation of the Hilbert series of the Coulomb branch of the moduli space for the $3 d \mathcal{N}=4$ quiver theories. For the computation, we use the monopole formula prescribed in [57] which essentially counts the number of dressed monopole operators according to their conformal dimension. We refer the readers to [57] for the technical details of the formula and simply quote the result here:

$$
\mathrm{HS}_{C}(t)=\sum_{\vec{m}_{1}} \sum_{\vec{m}_{2}} \ldots \sum_{\vec{m}_{x}} t^{\Delta\left(\vec{m}_{1}, \ldots, \vec{m}_{x}\right)} \prod_{i=1}^{x} P_{G_{i}}\left(t, \vec{m}_{i}\right) .
$$


Let us briefly explain various terms in this formula. The gauge group of the theory under consideration is $G_{1} \times G_{2} \times \ldots \times G_{x}$, where each of the group $G_{i}$ is indicated as a circular node in the quiver description. For a particular group $G$ in the quiver, the monopole operators are specified by the magnetic fluxes $\vec{m}=\left(m_{1}, m_{2}, \ldots m_{r}\right)$ which belong to the weight lattice $\Gamma(\hat{G})$ of $\hat{G}$, the GNO (or Langlands) dual group of $G$ ( $r$ being the rank of $G$ or $\hat{G}$ ). The gauge invariant monopole operators are specified by those $\vec{m}$ which take values in the quotient space:

$$
\vec{m} \in \Gamma(\hat{G}) / W(\hat{G}),
$$

where $W(\hat{G})$ is the Weyl group of $\hat{G}$. These are precisely the fluxes which contribute in the summation in the monopole formula (A.1). The Langland duals and the associated magnetic fluxes for some of the Lie groups are given below:

\begin{tabular}{|c|c|c|}
\hline$G$ & $\hat{G}$ & $\vec{m} \in \Gamma(\hat{G}) / W(\hat{G})$ \\
\hline $\mathrm{U}(r)$ & $\mathrm{U}(r)$ & $m_{1} \geq m_{2} \geq \ldots \geq m_{r} \geq-\infty$ \\
$\mathrm{SO}(2 r+1)$ & $\mathrm{USp}(2 r)$ & $m_{1} \geq m_{2} \geq \ldots \geq m_{r} \geq 0$ \\
$\mathrm{USp}(2 r)$ & $\mathrm{SO}(2 r+1)$ & $m_{1} \geq m_{2} \geq \ldots \geq m_{r} \geq 0$ \\
$\mathrm{SO}(2 r)$ & $\mathrm{SO}(2 r)$ & $m_{1} \geq m_{2} \geq \ldots \geq\left|m_{r}\right| \geq 0$ \\
\hline
\end{tabular}

The factor $P_{G}(t, \vec{m})$ is the classical dressing function which counts the gauge invariants of the residual gauge group which is left unbroken by the magnetic flux $\vec{m}$, according to their dimension, and is given as,

$$
P_{G}(t, \vec{m})=\prod_{i=1}^{r} \frac{1}{1-t^{d_{i}(\vec{m})}},
$$

where $d_{i}(\vec{m})$ are the degrees of Casimir invariants of the unbroken residual gauge group. These functions can be written in a computationally friendly manner by collecting the fluxes which are equal in $\vec{m}$. To do this, let us define an auxiliary sequence of non-increasing fluxes in $\vec{m}$, which we shall denote as $\vec{n}=\left(n_{1}, n_{2}, \ldots, n_{r}\right)$. We collect all the repeating fluxes together and define $\vec{n}_{\text {res }}=\left(a_{1}^{r_{1}}, \ldots, a_{u}^{r_{u}}\right)$ where the notation $a_{i}^{r_{i}}$ means that the integer $a_{i}$ is repeated $r_{i}$ times (where $r_{1}+\ldots+r_{u}=r$ ). The dressing functions can now be defined for various groups as:

\begin{tabular}{|c|c|c|c|}
\hline$G$ & $\vec{n}=\left(n_{1}, n_{2}, \ldots, n_{r}\right)$ & $\vec{n}_{\mathrm{res}}$ & $P_{G}$ \\
\hline $\mathrm{U}(r)$ & $\left(m_{1}, m_{2}, \ldots, m_{r}\right)$ & $\left(a_{1}^{r_{1}}, \ldots, a_{u}^{r_{u}}\right)$ & $\prod_{i=1}^{u} \prod_{k=1}^{r_{i}} \frac{1}{1-t^{k}}$ \\
\hline $\mathrm{SO}(2 r+1)$ & $\left(m_{1}, m_{2}, \ldots, m_{r}\right)$ & $\left(a_{1}^{r_{1}}, \ldots, a_{u-1}^{r_{u-1}}, 0^{r_{u}}\right)$ & $A\left(r_{u}\right)\left(\prod_{i=1}^{u-1} \prod_{k=1}^{r_{i}} \frac{1}{1-t^{k}}\right)$ \\
\hline $\mathrm{USp}(2 r)$ & $\left(m_{1}, m_{2}, \ldots, m_{r}\right)$ & $\left(a_{1}^{r_{1}}, \ldots, a_{u-1}^{r_{u-1}}, 0^{r_{u}}\right)$ & $A\left(r_{u}\right)\left(\prod_{i=1}^{u-1} \prod_{k=1}^{r_{i}} \frac{1}{1-t^{k}}\right)$ \\
\hline $\mathrm{SO}(2 r)$ & $\left(m_{1}, m_{2}, \ldots,\left|m_{r}\right|\right)$ & $\left(a_{1}^{r_{1}}, \ldots, a_{u-1}^{r_{u-1}}, 0^{r_{u}}\right)$ & $B\left(r_{u}\right)\left(\prod_{i=1}^{u-1} \prod_{k=1}^{r_{i}} \frac{1}{1-t^{k}}\right)$ \\
\hline
\end{tabular}

where the factors $A\left(r_{u}\right)$ and $B\left(r_{u}\right)$ are explicitly dependent on the number of vanishing fluxes and are given as,

$$
A(j)=\prod_{k=1}^{j} \frac{1}{1-t^{2 k}} ; \quad B(j)=\delta_{j 0}+\left(\frac{1}{1-t^{j}} \prod_{k=1}^{j-1} \frac{1}{1-t^{2 k}}\right)\left(1-\delta_{j 0}\right) .
$$


The last thing we need in the monopole formula (A.1) is the $R$-charge or the conformal dimension $\Delta\left(\vec{m}_{1}, \ldots, \vec{m}_{x}\right)$ of the bare monopole operators associated with various gauge groups in the quiver specified by the GNO magnetic fluxes $\vec{m}_{1}, \ldots, \vec{m}_{x}$. The conformal dimension gets contribution from the vector multiplets and the hyper multiplets present in the theory:

$$
\Delta=\Delta_{\text {vector }}+\Delta_{\text {hyper }} .
$$

These contributions are given as follows. Consider a node with group $G$ in the quiver and denote $\alpha$ to be a positive root of $G$. The vector contribution is computed as,

$$
\Delta_{\text {vector }}(\vec{m})=-\sum_{\alpha \in \Delta_{+}}|\alpha(\vec{m})|,
$$

where the positive roots of $G$ act on the GNO fluxes $\vec{m}$ associated with the weight lattice of GNO dual group $\hat{G}$ and the sum is taken over all positive roots of $G$. By restricting these fluxes to the fundamental Weyl chamber, the above sum can be explicitly performed and is given as,

\begin{tabular}{|c|c|c|}
\hline$G$ & $\Delta_{+}(G)$ & $\Delta_{\text {vector }}(\vec{m})$ \\
\hline $\mathrm{U}(r)$ & $\left\{e_{i}-e_{j}\right\}_{1 \leq i<j \leq r}$ & $-\sum_{k=1}^{r}(r+1-2 k) m_{k}$ \\
$\mathrm{SO}(2 r+1)$ & $\left\{e_{i}-e_{j}, e_{i}+e_{j}, e_{i}\right\}_{1 \leq i<j \leq r}$ & $-\sum_{k=1}^{r}(2 r+1-2 k) m_{k}$ \\
$\mathrm{USp}(2 r)$ & $\left\{e_{i}-e_{j}, e_{i}+e_{j}, 2 e_{i}\right\}_{1 \leq i<j \leq r}$ & $-\sum_{k=1}^{r}(2 r+2-2 k) m_{k}$ \\
$\mathrm{SO}(2 r)$ & $\left\{e_{i}-e_{j}, e_{i}+e_{j}\right\}_{1 \leq i<j \leq r}$ & $-\sum_{k=1}^{r-1}(2 r-2 k) m_{k}$ \\
\hline
\end{tabular}

Once we compute this vector term for individual nodes in the quiver, we can simply add them to get the full vector contribution:

$$
\Delta_{\text {vector }}\left(\vec{m}_{1}, \ldots, \vec{m}_{x}\right)=\sum_{i=1}^{x} \Delta_{\text {vector }}\left(\vec{m}_{i}\right) .
$$

The term $\Delta_{\text {hyper }}$ is the contribution of hypermultiplets (the links connecting the nodes in the quiver diagram) present in the theory which is given as the sum over the weights of the matter field representation under the gauge groups. To write an explicit formula in terms of the GNO fluxes, consider a hypermultiplet connecting two groups $G_{r}$ and $G_{s}$ of ranks $r$ and $s$ :

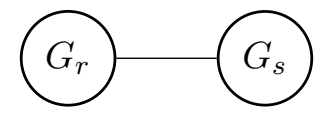

Further consider the GNO fluxes associated with the two groups as $\vec{a}=\left(a_{1}, a_{2}, \ldots, a_{r}\right)$ and $\vec{b}=\left(b_{1}, b_{2}, \ldots, b_{s}\right)$. The weights associated with the fundamental representation of the two groups can be written as a tuple of GNO fluxes which we denote as $w(\vec{a})$ and $w(\vec{b})$. For the classical Lie groups, they are given below:

\begin{tabular}{|c|c|c|}
\hline$G$ & $\vec{m}$ & $w(\vec{m})$ \\
\hline $\mathrm{U}(r)$ & $\left(m_{1}, \ldots, m_{r}\right)$ & $\left(m_{1}, \ldots, m_{r}\right)$ \\
$\mathrm{SO}(2 r+1)$ & $\left(m_{1}, \ldots, m_{r}\right)$ & $\left(m_{1}, \ldots, m_{r}, 0,-m_{r}, \ldots,-m_{1}\right)$ \\
$\mathrm{USp}(2 r)$ & $\left(m_{1}, \ldots, m_{r}\right)$ & $\left(m_{1}, \ldots, m_{r},-m_{r}, \ldots,-m_{1}\right)$ \\
$\mathrm{SO}(2 r)$ & $\left(m_{1}, \ldots, m_{r}\right)$ & $\left(m_{1}, \ldots, m_{r},-m_{r}, \ldots,-m_{1}\right)$ \\
\hline
\end{tabular}


The hypermulitplet contribution is now easy to write. If we denote $w_{i}(\vec{m})$ to be the $i^{\text {th }}$ component in $w(\vec{m})$, the contribution is:

$$
\Delta_{\text {hyper }}(\vec{a}, \vec{b})=\left\{\begin{array}{ll}
\frac{1}{4} \sum_{i} \sum_{j}\left|w_{i}(\vec{a})-w_{j}(\vec{b})\right|, & \text { when } G_{r} / G_{s}=\text { SO/USp or USp/SO } \\
\frac{1}{2} \sum_{i} \sum_{j}\left|w_{i}(\vec{a})-w_{j}(\vec{b})\right|, & \text { otherwise }
\end{array},\right.
$$

where a hypermultiplet comes with a $1 / 2$ factor and a half-hypermultiplet (the link connecting a SO type of node to USp type of node) comes with a $1 / 4$ factor in the conformal dimension. Summing over the contributions of all the hypermultiplets present in the theory will finally give the $\Delta_{\text {hyper }}$ for the full quiver. There can also be flavor symmetry groups associated with a gauge group. Such groups are denoted by a square node in the quiver. The GNO fluxes for such nodes are all $0(\vec{m}=0)$ and they do not contribute to $\Delta_{\text {vector }}$ in the conformal dimension. The contribution of the link connecting the gauge node with a flavor node can be obtained by simply computing $\Delta_{\text {hyper }}$ as usual and then setting the fluxes of GNO dual to 0:

$$
G_{r} \quad G_{s} \Longrightarrow \Delta_{\text {hyper }}=\Delta_{\text {hyper }}(\vec{a}, \overrightarrow{0}) \text {. }
$$

We may also encounter the cases where we need to put multiple links between two nodes in the quiver. In this scenario, the contribution of the individual links are simply added up. For example, in case of triple hypers, we have:

$$
G_{r} \Longrightarrow \Delta_{s} \Longrightarrow \Delta_{\text {hyper }}=3 \times \Delta_{\text {hyper }}(\vec{a}, \vec{b}) \text {. }
$$

We have also proposed an exotic hypermultiplet which transforms under the fundamentalfundamental representations of the unitary gauge nodes it connects. We denote this exotic hyper by a dashed line in the quiver between the two unitary nodes. The contribution of such a hyper to the conformal dimension is given as:

$$
\text { U(r)-- U }(s) \Longrightarrow \Delta_{\text {hyper }}=\frac{1}{2} \sum_{i=1}^{r} \sum_{j=1}^{s}\left|w_{i}(\vec{a})+w_{j}(\vec{b})\right|,
$$

where $\vec{a}$ and $\vec{b}$ are the GNO fluxes of the two nodes.

\section{A.2 Higgs branch Hilbert series}

The Hilbert series of the Higgs branch of a $3 \mathrm{~d} \mathcal{N}=4$ theory can be easily computed. Suppose the gauge group is $G$, and the matter content is given by $N_{h}$ hypermultiplets charged under representations $R_{i}\left(i=1, \ldots, N_{h}\right)$ of the gauge group, and possibly charged under representations $R_{i}^{\prime}$ of the flavor group. The Hilbert series is then schematically given by

$$
\mathrm{HS}(t)=\int_{G} d \mu_{G} \operatorname{Pfc}(w, t) \operatorname{PE}\left[\sum_{i=1}^{2 N_{h}} \operatorname{char}_{R_{i}}(w) \operatorname{char}_{R_{i}^{\prime}}(x) t^{\frac{1}{2}}\right]
$$

Let us now review the various terms entering in equation (A.17). 
The term

$$
\mathrm{PE}\left[\sum_{i=1}^{2 N_{h}} \operatorname{char}_{R_{i}}(w) \operatorname{char}_{R_{i}^{\prime}}(x) t^{\frac{1}{2}}\right]
$$

is generating all the possible symmetrized product of all the scalars inside the hypermultiplets. Here $w=\left(w_{1}, \cdots, w_{r}\right)$ is a collective variable denoting the fugacities of the rank $r$ gauge group, and $x=\left(x_{1}, \cdots, x_{r}\right)$ are fugacities of the flavor group. Notice that these fugacities take value in the gauge (resp. flavor) symmetry group. Therefore each of the $x$ and $w$ is a complex phase. The fugacity $t$ is a fugacity counting the conformal dimension. The exponent of $t$ is $\frac{1}{2}$ as this is the conformal dimension of one free scalar in $3 \mathrm{~d}$. The term $\operatorname{char}_{R_{i}}(w)$ (resp. $\operatorname{char}_{R i_{i}}(x)$ ) is the character of the representation under the gauge group (resp. flavor group) under which the $i$-th hypermultiplet is charged. The function PE[.] is the plethystic exponential, defined as

$$
\operatorname{PE}[f(x)]:=\exp \left(\sum_{k=1}^{\infty} \frac{1}{k} f\left(x^{k}\right)\right)
$$

for any function $f(x)$ such that $f(0)=0$. The term $\operatorname{Pfc}(w, t)$ is a prefactor, encoding the fact that the symmetrized product of the scalars are not all independent, but they obey some relationships coming from the F-term constraints. This prefactor term is given by

$$
\operatorname{Pfc}(w, t)=\operatorname{PE}\left[\sum_{i=1}^{N_{r}} \operatorname{char}_{R_{i}^{\prime \prime}}(w) t^{d_{i}}\right]^{-1}
$$

where $\operatorname{char}_{R_{i}^{\prime \prime}}(w)$ is the character of the representation of the $i$-th relation, and $d_{i}$ its degree in conformal dimension. Given the constrained structure of the superpotential in theories with 8 supercharges, typically $\operatorname{char}_{R_{i}^{\prime \prime}}(w)$ will be the character of the adjoint, and the relation will appear at quadratic order in the scalars: $d_{i}=1$. Finally, the integral over the gauge group is performed in order to count only gauge invariant operators, and not just all the symmetric products of scalars modulo the F-term constraint. Such integral is called the Molien integral. The integration measure $\mu_{G}$ is given, for any continuous gauge group as

$$
\int_{G} d \mu_{G}=\frac{1}{(2 \pi i)^{r}} \oint_{|w|_{1}=1} \cdots \oint_{|w|_{r}=1} \frac{d w_{1}}{w_{1}} \cdots \frac{d w_{r}}{w_{r}} \prod_{\alpha \in \Delta^{+}}\left(1-\prod_{k=1}^{r} w_{k}^{\alpha_{k}}\right)
$$

where $\Delta^{+}$is the set of the positive roots of the Lie algebra of $G$. Notice that despite the integral is formally performed over the full gauge group, the use of the Molien measure localizes the integral just on the Cartan torus. Every fugacity $w_{i}$ is then integrated just on the unit circle. The factor $\frac{d w_{r}}{w_{r}} \prod_{\alpha \in \Delta^{+}}\left(1-\prod_{k=1}^{r} w_{k}^{\alpha_{k}}\right)$ can be thought of the Jacobian for the change of variable recasting the integral over the gauge group into the integral over the its Cartan torus.

The procedure outlined above can be slightly modified if discrete gauge groups are present. Let us call the discrete gauge group $H$, and its order $|H|$. We can introduce a 
fugacity $z$ for the discrete gauge symmetry, valued now in $H$. The term generating all the symmetrized products of scalars will now read

$$
\mathrm{PE}\left[\sum_{i=1}^{2 N_{h}} \operatorname{char}_{R_{i}}(w) \operatorname{char}_{R_{i}^{\prime}}(x) z t^{\frac{1}{2}}\right]
$$

and there will be no prefactor contribution. This is consistent with the fact that there is no vector multiplet associated to a discrete gauge factor, therefore there will be no F-term relations for it. To only retain the singlets for the discrete factor, the Molien integral over $H$ still has to be performed. However, since now the $z$ fugacity takes values is a discrete group, the integral over all the elements of the gauge group is now replaced with discrete sum:

$$
\begin{aligned}
& \int_{H} d \mu_{H} \operatorname{Pfc}(w, t) \operatorname{PE}\left[\sum_{i=1}^{2 N_{h}} \operatorname{char}_{R_{i}}(w) \operatorname{char}_{R_{i}^{\prime}}(x) z t^{\frac{1}{2}}\right]= \\
& =\frac{1}{|H|} \operatorname{Pfc}(w, t) \sum_{h}^{|H|} \operatorname{PE}\left[\sum_{i=1}^{2 N_{h}} \operatorname{char}_{R_{i}}(w) \operatorname{char}_{R_{i}^{\prime}}(x) z t^{\frac{1}{2}}\right]_{\mid z=h}
\end{aligned}
$$

After performing all the discrete Molien integrations for all the discrete factors of the gauge group, the integrations on the continuous factors still have to be performed, using eq. (A.21).

\section{B Palindromic polynomials}

We summarize in table 18 explicit forms of parlindromic polynomials which arise in the computation of the Hilbert series of Coulomb branches of some theories discussed in section 2 and section 3 . 


\begin{tabular}{|c|c|}
\hline Label & Polynomial \\
\hline$P_{0}(t)$ & $\begin{array}{c}1+98 t+3312 t^{2}+53305 t^{3}+468612 t^{4}+2421286 t^{5}+7664780 t^{6}+15203076 t^{7}+19086400 t^{8}+ \\
15203076 t^{9}+7664780 t^{10}+2421286 t^{11}+468612 t^{12}+53305 t^{13}+3312 t^{14}+98 t^{15}+t^{16}\end{array}$ \\
\hline$P_{1}(t)$ & $\begin{array}{c}1+52 t+2669 t^{2}+63963 t^{3}+1011274 t^{4}+11000351 t^{5}+88762624 t^{6}+549522302 t^{7}+ \\
2698733098 t^{8}+10730180908 t^{9}+35179028314 t^{10}+96291284692 t^{11}+222448351508 t^{12}+ \\
436990904921 t^{13}+734668194786 t^{14}+1061421775571 t^{15}+1322191150030 t^{16}+ \\
1422261110352 t^{17}+\ldots \text { palindrome } \ldots+52 t^{33}+t^{34}\end{array}$ \\
\hline$P_{2}(t)$ & $\begin{array}{c}64 t+2560 t^{2}+64768 t^{3}+1006592 t^{4}+11022656 t^{5}+88672768 t^{6}+549832576 t^{7}+2697805824 t^{8}+ \\
10732603264 t^{9}+35173452800 t^{10}+96302654592 t^{11}+222427719168 t^{12}+437024347328 t^{13}+ \\
734619634688 t^{14}+1061485070080 t^{15}+1322116993536 t^{16}+1422339277952 t^{17}+ \\
\ldots \text { palindrome } \ldots+2560 t^{32}+64 t^{33}\end{array}$ \\
\hline$P_{3}(t)$ & $1+31 t+231 t^{2}+595 t^{3}+595 t^{4}+231 t^{5}+31 t^{6}+t^{7}$ \\
\hline$P_{4}(t)$ & $\begin{array}{c}1+22 t+245 t^{2}+1442 t^{3}+5355 t^{4}+12978 t^{5}+21919 t^{6}+25900 t^{7}+21919 t^{8}+12978 t^{9}+5355 t^{10}+ \\
1442 t^{11}+245 t^{12}+22 t^{13}+t^{14}\end{array}$ \\
\hline$P_{5}(t)$ & $1+14 t+91 t^{2}+336 t^{3}+819 t^{4}+1362 t^{5}+1618 t^{6}+1362 t^{7}+819 t^{8}+336 t^{9}+91 t^{10}+14 t^{11}+t^{12}$ \\
\hline$P_{6}(t)$ & $1+12 t+58 t^{2}+124 t^{3}+170 t^{4}+124 t^{5}+58 t^{6}+12 t^{7}+t^{8}$ \\
\hline$P_{7}(t)$ & $8 t+48 t^{2}+136 t^{3}+176 t^{4}+136 t^{5}+48 t^{6}+8 t^{7}$ \\
\hline$P_{8}(t)$ & $1+6 t+44 t^{2}+146 t^{3}+446 t^{4}+826 t^{5}+1343 t^{6}+1436 t^{7}+1343 t^{8}+826 t^{9}+446 t^{10}+146 t^{11}+44 t^{12}+6 t^{13}+t^{14}$ \\
\hline$P_{9}(t)$ & $\begin{array}{c}1+6 t+35 t^{2}+108 t^{3}+407 t^{4}+1014 t^{5}+2720 t^{6}+5198 t^{7}+10773 t^{8}+16712 t^{9}+27493 t^{10}+ \\
35046 t^{11}+47571 t^{12}+50460 t^{13}+56752 t^{14}+50640 t^{15}+47571 t^{16}+35046 t^{17}+27493 t^{18}+ \\
16712 t^{19}+10773 t^{20}+5198 t^{21}+2720 t^{22}+1014 t^{23}+407 t^{24}+108 t^{25}+35 t^{26}+6 t^{27}+t^{28}\end{array}$ \\
\hline$P_{10}(t)$ & $\begin{array}{c}16 t^{2}+80 t^{3}+368 t^{4}+960 t^{5}+2704 t^{6}+5296 t^{7}+10912 t^{8}+16832 t^{9}+27728 t^{10}+35184 t^{11}+ \\
47344 t^{12}+50192 t^{13}+56608 t^{14}+50192 t^{15}+47344 t^{16}+35184 t^{17}+27728 t^{18}+16832 t^{19}+ \\
10912 t^{20}+5296 t^{21}+2704 t^{22}+960 t^{23}+368 t^{24}+80 t^{25}+16 t^{26}\end{array}$ \\
\hline$P_{11}(t)$ & $1+11 t+57 t^{2}+170 t^{3}+324 t^{4}+398 t^{5}+324 t^{6}+170 t^{7}+57 t^{8}+11 t^{9}+t^{10}$ \\
\hline$P_{12}(t)$ & $1+3 t+38 t^{2}+69 t^{3}+225 t^{4}+240 t^{5}+372 t^{6}+240 t^{7}+225 t^{8}+69 t^{9}+38 t^{10}+3 t^{11}+t^{12}$ \\
\hline$P_{13}(t)$ & $8 t+20 t^{2}+112 t^{3}+156 t^{4}+328 t^{5}+276 t^{6}+328 t^{7}+156 t^{8}+112 t^{9}+20 t^{10}+8 t^{11}$ \\
\hline$P_{14}(t)$ & $1+2 t+6 t^{2}+10 t^{3}+22 t^{4}+26 t^{5}+39 t^{6}+36 t^{7}+39 t^{8}+26 t^{9}+22 t^{10}+10 t^{11}+6 t^{12}+2 t^{13}+t^{14}$ \\
\hline$P_{15}(t)$ & $\begin{array}{c}1-2 t^{2}+8 t^{5 / 2}+t^{3}-8 t^{7 / 2}+t^{4}-8 t^{9 / 2}+13 t^{5}+8 t^{11 / 2}-24 t^{6}-5 t^{7}+8 t^{15 / 2}+15 t^{8}-24 t^{17 / 2}-t^{9}+ \\
8 t^{19 / 2}+9 t^{10}+8 t^{21 / 2}-16 t^{11}+8 t^{23 / 2}+9 t^{12}+8 t^{25 / 2}-t^{13}-24 t^{27 / 2}+15 t^{14}+8 t^{29 / 2}-5 t^{15}- \\
24 t^{16}+8 t^{33 / 2}+13 t^{17}-8 t^{35 / 2}+t^{18}-8 t^{37 / 2}+t^{19}+8 t^{39 / 2}-2 t^{20}+t^{22}\end{array}$ \\
\hline$P_{16}(t)$ & $\begin{array}{c}1-2 t^{2}+t^{3}+t^{4}+16 t^{5}-24 t^{6}-11 t^{7}+18 t^{8}+2 t^{9}+51 t^{10}-88 t^{11}-12 t^{12}+47 t^{13}+15 t^{14}+62 t^{15}- \\
144 t^{16}+23 t^{17}+44 t^{18}+44 t^{19}+23 t^{20}-144 t^{21}+62 t^{22}+15 t^{23}+47 t^{24}-12 t^{25}-88 t^{26}+51 t^{27}+ \\
2 t^{28}+18 t^{29}-11 t^{30}-24 t^{31}+16 t^{32}+t^{33}+t^{34}-2 t^{35}+t^{37}\end{array}$ \\
\hline$P_{17}(t)$ & $\begin{array}{c}1-t-t^{2}+t^{3}+4 t^{5}-6 t^{6}-2 t^{7}+4 t^{8}+t^{9}+7 t^{10}-15 t^{11}+t^{12}+6 t^{13}+4 t^{14}+6 t^{15}-20 t^{16}+6 t^{17}+ \\
4 t^{18}+6 t^{19}+t^{20}-15 t^{21}+7 t^{22}+t^{23}+4 t^{24}-2 t^{25}-6 t^{26}+4 t^{27}+t^{29}-t^{30}-t^{31}+t^{32}\end{array}$ \\
\hline$P_{18}(t)$ & $1+8 t+40 t^{2}+107 t^{3}+199 t^{4}+234 t^{5}+199 t^{6}+107 t^{7}+40 t^{8}+8 t^{9}+t^{10}$ \\
\hline$P_{19}(t)$ & $1+2 t+6 t^{2}+10 t^{3}+22 t^{4}+26 t^{5}+39 t^{6}+36 t^{7}+39 t^{8}+26 t^{9}+22 t^{10}+10 t^{11}+6 t^{12}+2 t^{13}+t^{14}$ \\
\hline
\end{tabular}

Table 18. Palindromic polynomials appearing in the main sections. 


\section{More on the number of charge 2 hypermultiplets}

In section 2.2 , we observed that the number of charge 2 hypermultiplets may be given by (2.6). So far, we have encountered the cases which involve a $(p, 1) 5$-brane or a $(1, q)$ 5 -brane for the origin of charge 2 hypermultiplets. We here give more support for (2.6) by checking further new examples that involve a $(p, q) 5$-brane with both $p$ and $q$ larger than 1 . In order to consider such examples, we use 5-brane web diagrams whose corresponding orthosymplectic magnetic quiver theories do not have a unitary counterpart. However, we can still check the validity of the $3 \mathrm{~d}$ orthosymplectic magnetic quivers by comparing their Higgs branch dimension with the dimension of the Coulomb branch moduli space of the original $5 \mathrm{~d}$ theories.

The first example we consider is the $5 \mathrm{~d}$ theory realized on the 5 -brane web diagram in figure 44. The central gauge theory is an $\mathrm{SO}(4 k+2)$ gauge theory and the diagram has an external $(2 k-1,2) 5$-brane and an external $(2 k-1,-2) 5$-brane. The parts surrounded by the dashed circles need to be properly resolved and it introduces $k-1$ faces for each part. Then the dimension of the Coulomb branch moduli space of the $5 d$ theory can be counted by the number of faces in the diagram and it is

$$
\operatorname{dim} \mathcal{M}_{C}=2 k+1+2+2(k-1)=4 k+1 .
$$

At the infinitely strong coupling the diagram becomes the one in figure 45 . The brane web has $(2 k-1,2) 5$-brane and the number of the charge 2 hypermultiplets coupled to the $\mathrm{U}(1)$ gauge node originated from the 5-brane is

$$
\frac{8 k-4}{2}-2=4 k-4
$$

according to (2.6). Namely the orientifold web diagram in figure 45 yields the orthosymplectic magnetic quiver depicted in (C.3).



For counting the dimension of the Higgs branch moduli space of the theory in (C.3), we compute the number of hypermultiplets and the number of vector multiplets and then do subtraction. The number of hypermultipltes is

$$
H=2+4+4+2+8+4 k-4=16+4 k,
$$

and the number of vector multiplets is

$$
V=1+3+6+3+1+1=15 .
$$




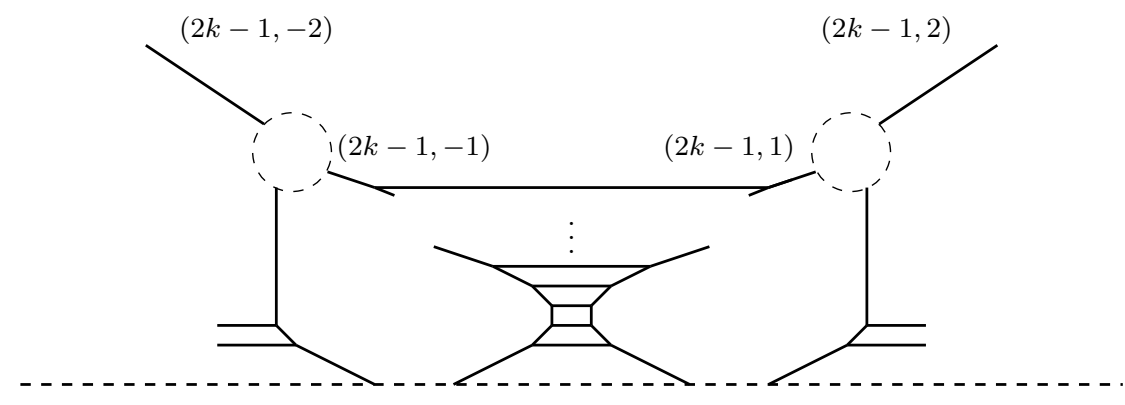

O5

O5

Figure 44. The 5-brane web diagram which has an external $(2 k-1,2) 5$-brane and an external $(2 k-1,-2) 5$-brane.

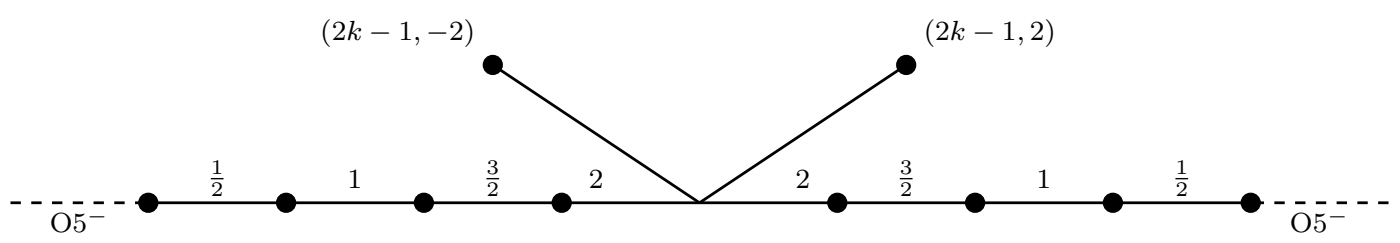

Figure 45. The orientifold web diagram for the theory given in figure 44 at the infinitely strong coupling.

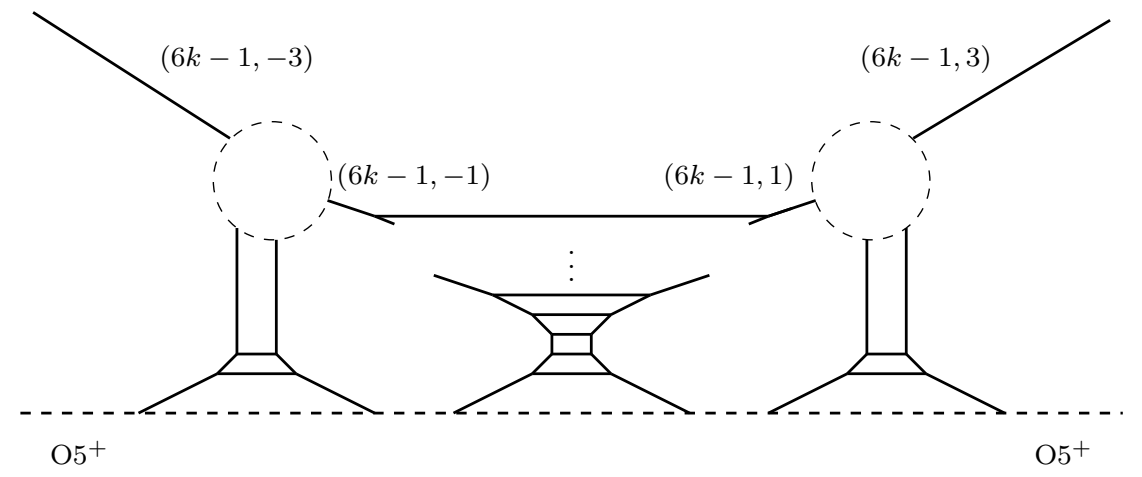

Figure 46. The 5-brane web diagram which has an external $(6 k-1,3) 5$-brane and an external $(6 k-1,-3) 5$-brane.

Hence the dimension of the Higgs branch is given by

$$
\operatorname{dim}_{\mathbb{H}} \mathcal{M}_{H}=16+4 k-15=4 k+1
$$

We find that (C.6) is exactly the same as (C.1) and this gives support for the number of charge 2 hypermultiplets counting in (C.2).

The first example is in the class of $05^{-}-\mathrm{O} 5^{-}$and let us also consider an example in the class of $\mathrm{O} 5^{+}-\mathrm{O}^{+}$. The second example we choose is the $5 \mathrm{~d}$ theory realized on the 5 -brane web diagram in figure 46 . The central gauge theory is now an $\operatorname{SO}(12 k+2)$ gauge theory and the diagram contains an external $(6 k-1,3) 5$-brane and an external $(6 k-1,-3) 5$-brane. The parts surrounded by the dashed circles again need to be resolved 


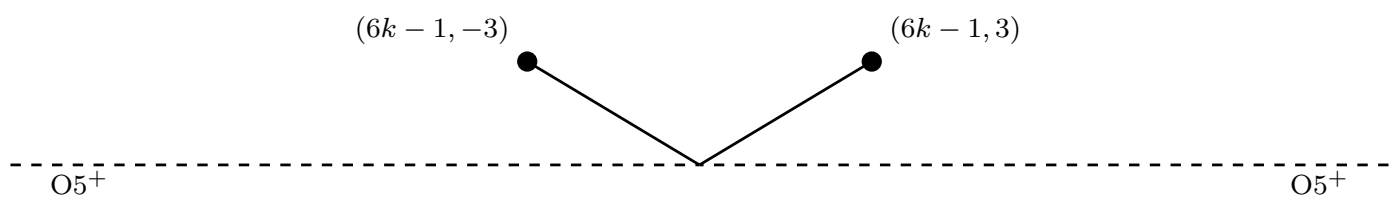

Figure 47. The orientifold web diagram for the theory given in figure 46 at the infinitely strong coupling.

and each part in fact has $6 k-2$ faces. In this case the dimension of the Coulomb branch moduli space of the $5 \mathrm{~d}$ theory is

$$
\operatorname{dim} \mathcal{M}_{C}=6 k+1+2+6+2(6 k-2)=18 k+5 .
$$

For reading off the magnetic quiver theory we take the infinitely strong coupling limit of the diagram in figure 46 and it becomes the one in figure 47 . The $(6 k-1,3) 5$-brane and the $(6 k-1,-3) 5$-brane yields a $\mathrm{U}(1)$ gauge theory and the number of charge 2 hypermultiplets computed by $(3.5)$ is

$$
\frac{6(3 k-1)}{2}-3=18 k-6
$$

Furthermore we also expect the presence of the $3 \times 4=12$ charge 1 hypermutiplets which is computed by using (3.6). Then the magentic quiver theory which arises from the diagram in figure 47 is given in (C.9).

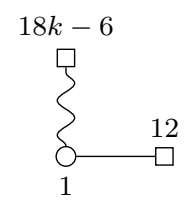

We can compute the dimension of the Higgs branch moduli space of the orthosymplectic magnetic quiver theory in (C.9) in a similar way. The number of hypermulitplets in the quiver is

$$
H=18 k-6+12=18 k+6
$$

and the number of vector multiplets is

$$
V=1
$$

which yields

$$
\operatorname{dim}_{\mathbb{H}} \mathcal{M}_{H}=18 k+5 .
$$

We again find the agreement with (C.7) which gives another support for (2.6). 


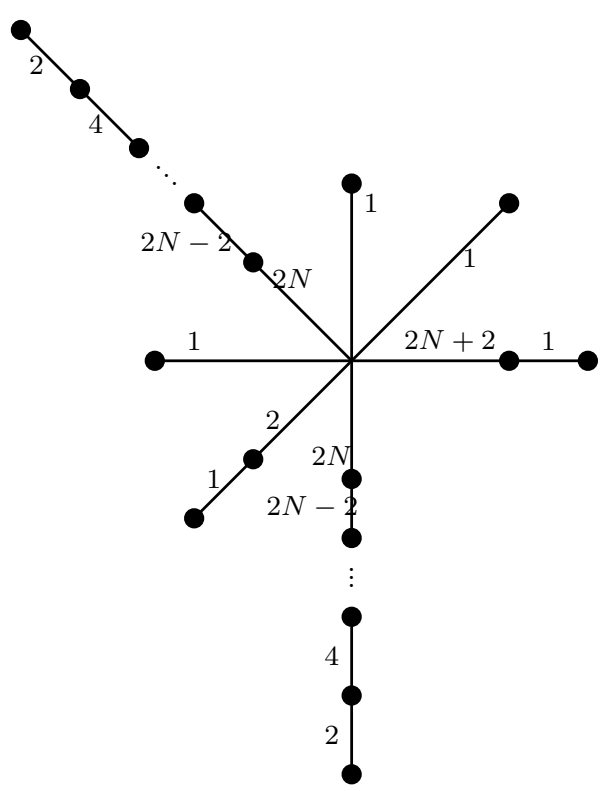

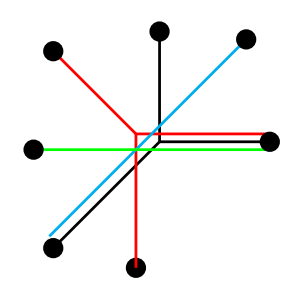

(I)

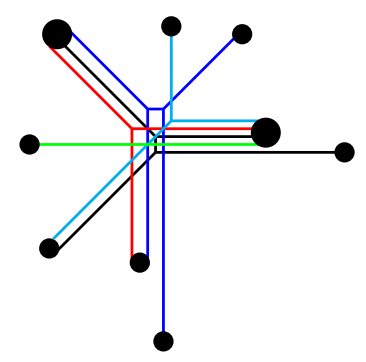

(III)

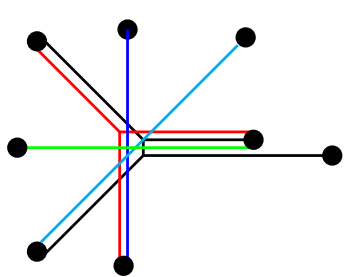

(II)



(IV)

Figure 48. Ordinary web for the $\mathrm{Y}_{N}^{2,1}$ theory along with its maximal subdivisions.

\section{The remaining theories in the $Y_{N}$ family}

There are a few more possible configurations in the $Y_{N}^{p, q}$ family. They do not give rise to any new rules, in addition to those already mentioned so far. They do however serve as working examples, that demonstrate the validity of the rules proposed earlier. Without delving into a technical discussion, we present the web diagrams along with the corresponding magnetic quivers for the $Y_{N}^{p, q}$ family. We have also checked the matching of the Coulomb branch Hilbert series for the unitary and orthosymplectic quivers for each of the family. These results have been presented in various figures and tables, as summarized in the following.

\begin{tabular}{|c|c|c|c|c|}
\hline Theory & Unitray web & Orientifold web & Magnetic quivers & Coulomb branch HS \\
$\mathrm{Y}_{N}^{2,1}$ & Figure 48 & Figure 49 & Table 19 & Table 20 \\
$\mathrm{Y}_{N}^{3,1}$ & Figure 50 & Figure 51 & Figure 52 & Table 21 \\
$\mathrm{Y}_{N}^{2,2}$ & Figure 53 & Figure 54 & Table 22 & Table 23 \\
$\mathrm{Y}_{N}^{2,3}$ & Figure 55 & Figure 56 & Figure 57 & Table 24 \\
\hline
\end{tabular}




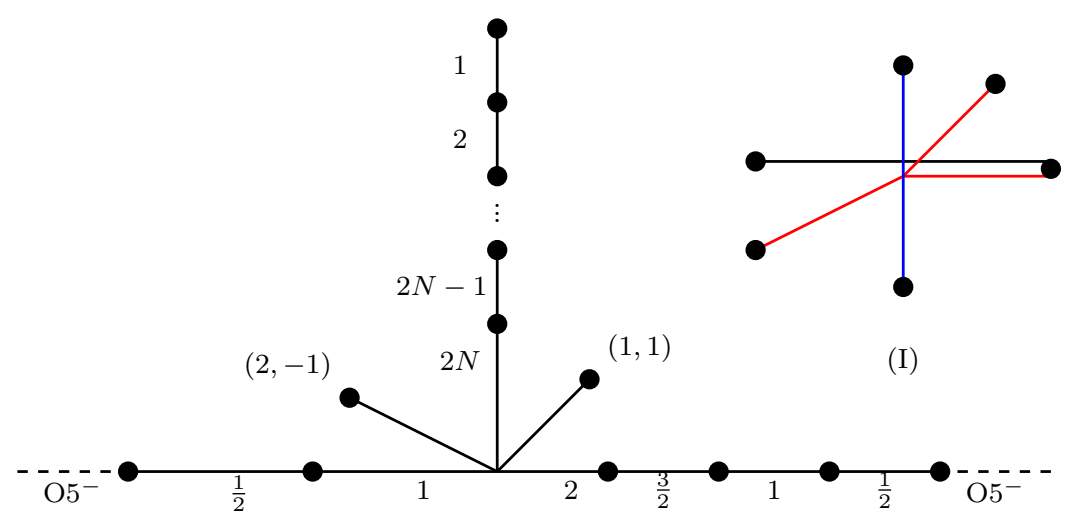

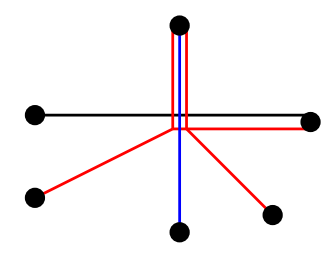

(II)

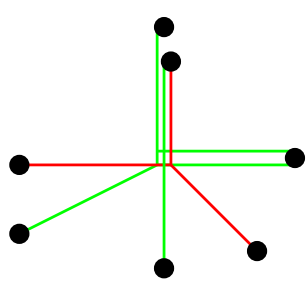

(III)

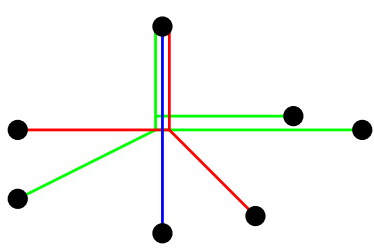

(IV)

Figure 49. Orientifold web for the $\mathrm{Y}_{N}^{2,1}$ theory along with its maximal subdivisions.
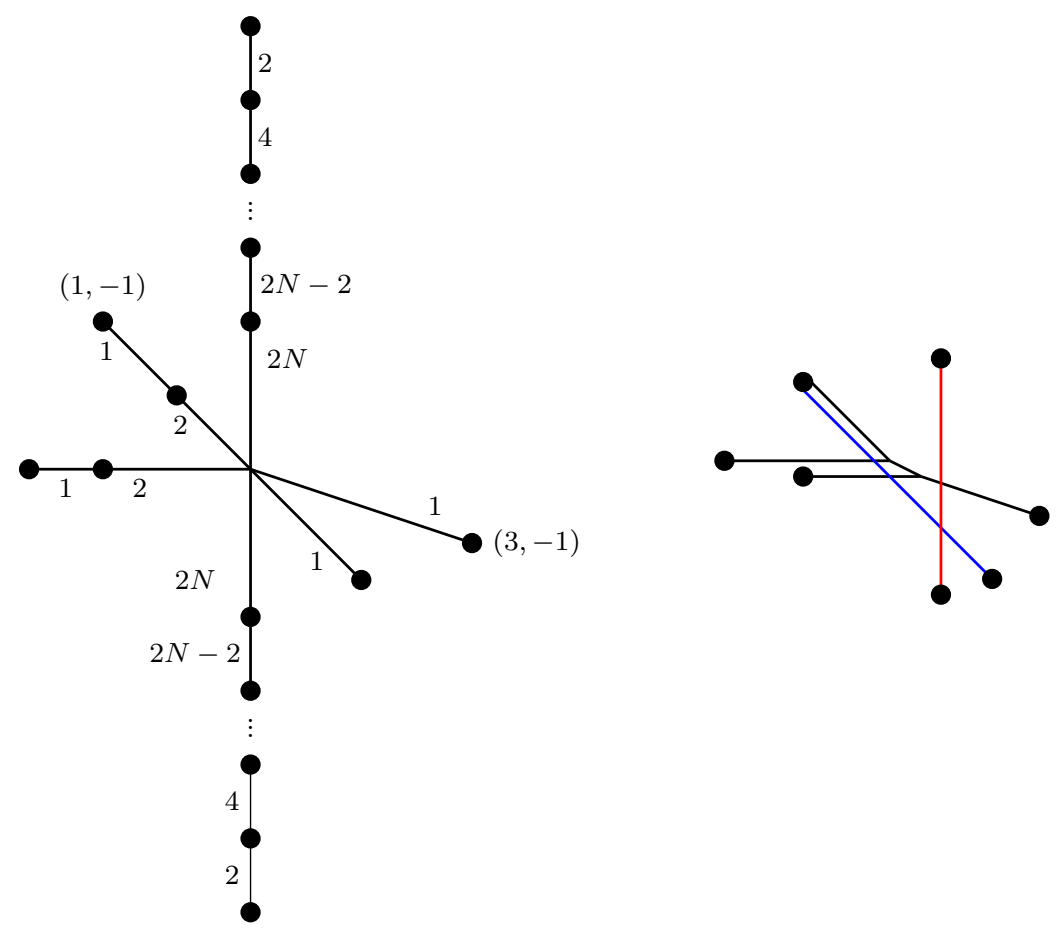

Figure 50. Ordinary web diagram for the $\mathrm{Y}_{N}^{3,1}$ theory along with the maximal subdivision for the centre of the junction. 


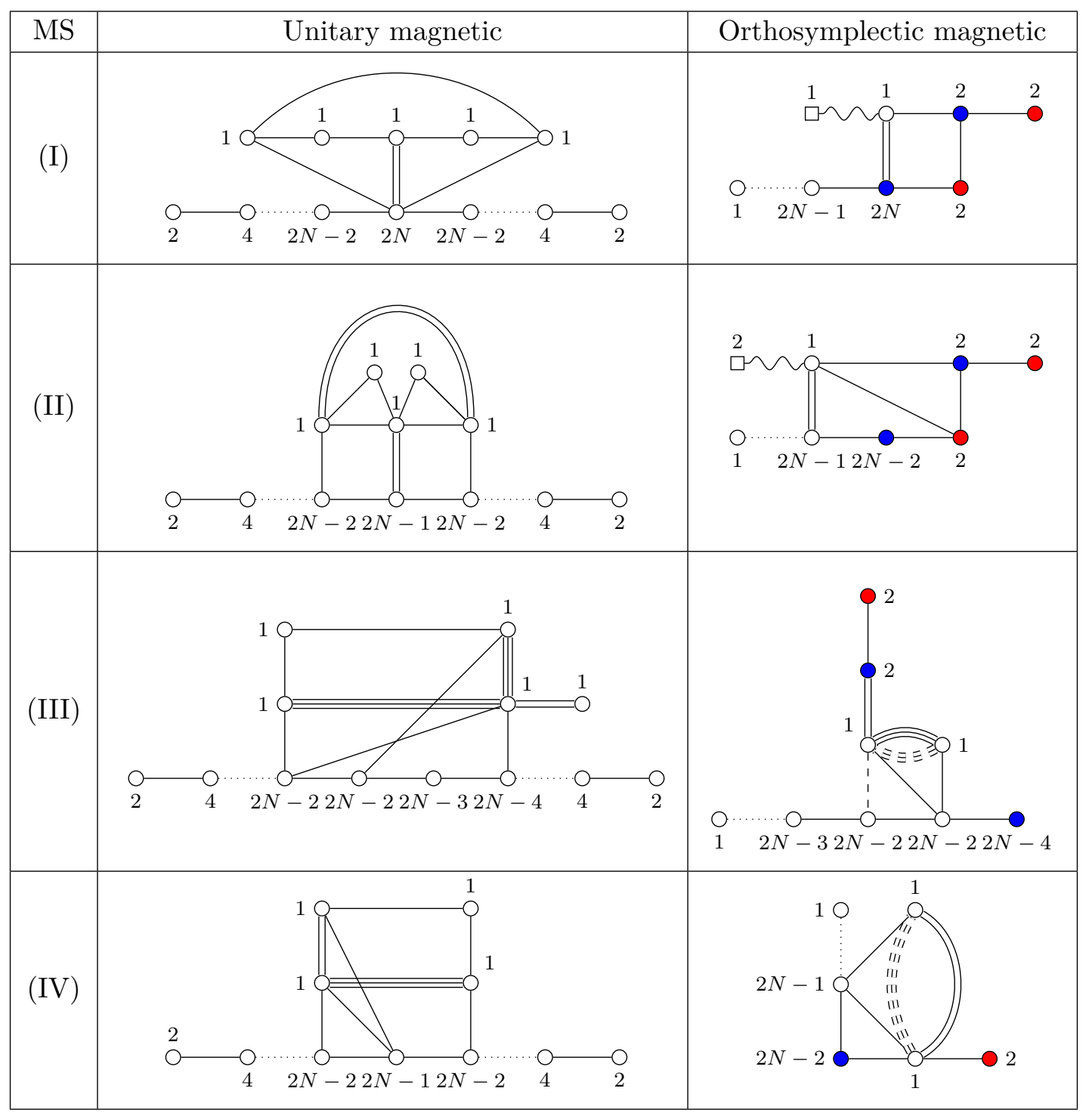

Table 19. The magnetic quivers derived from various maximal subdivisions (MS) of the unitary and orientifold webs of the $\mathrm{Y}_{N}^{2,1}$ theory. 


\begin{tabular}{|c|c|c|c|}
\hline \multirow{2}{*}{ MS } & Unitary & \multicolumn{2}{|c|}{ Orthosymplectic } \\
\hline & $\mathrm{HS}(t)$ & $\mathrm{HS}(t ; \vec{m} \in \mathbb{Z})$ & $\mathrm{HS}\left(t ; \vec{m} \in \mathbb{Z}+\frac{1}{2}\right)$ \\
\hline$(\mathrm{I})_{N=1}$ & $\begin{array}{c}1+11 t+91 t^{2}+552 t^{3}+ \\
2654 t^{4}+10598 t^{5}+\cdots\end{array}$ & $\begin{array}{c}1+11 t+75 t^{2}+392 t^{3}+ \\
1710 t^{4}+6422 t^{5}+\cdots\end{array}$ & $\begin{array}{c}16 t^{2}+160 t^{3}+944 t^{4}+ \\
4176 t^{5}+\cdots\end{array}$ \\
\hline$(\mathrm{I})_{N=2}$ & $\begin{array}{c}1+23 t+289 t^{2}+2638 t^{3}+ \\
19566 t^{4}+124453 t^{5}+\cdots\end{array}$ & $\begin{array}{c}1+23 t+289 t^{2}+2590 t^{3}+ \\
18510 t^{4}+111893 t^{5}+\cdots\end{array}$ & $48 t^{3}+1056 t^{4}+12560 t^{5}+\cdots$ \\
\hline$(\mathrm{II})_{N=1}$ & $\begin{array}{c}1+11 t+79 t^{2}+405 t^{3}+ \\
1644 t^{4}+5572 t^{5}+\cdots\end{array}$ & $\begin{array}{c}1+11 t+71 t^{2}+325 t^{3}+ \\
1196 t^{4}+3764 t^{5}+\cdots\end{array}$ & $\begin{array}{c}8 t^{2}+80 t^{3}+448 t^{4}+ \\
1808 t^{5}+\cdots\end{array}$ \\
\hline$(\mathrm{II})_{N=2}$ & $\begin{array}{c}1+23 t+289 t^{2}+2622 t^{3}+ \\
19178 t^{4}+119256 t^{5}+\cdots\end{array}$ & $\begin{array}{c}1+23 t+289 t^{2}+2590 t^{3}+ \\
18474 t^{4}+110936 t^{5}+\cdots\end{array}$ & $32 t^{3}+704 t^{4}+8320 t^{5}+$ \\
\hline$(\mathrm{III})_{N=2}$ & $\begin{array}{c}1+23 t+273 t^{2}+2255 t^{3}+ \\
14595 t^{4}+78621 t^{5}+\cdots\end{array}$ & $\begin{array}{c}1+23 t+273 t^{2}+2215 t^{3}+ \\
13795 t^{4}+70381 t^{5}+\cdots\end{array}$ & $40 t^{3}+800 t^{4}+8240 t^{5}+$ \\
\hline$(\mathrm{III})_{N=3}$ & $x x$ & $x x$ & $x x$ \\
\hline$(\mathrm{IV})_{N=1}$ & $\begin{array}{c}1+8 t+46 t^{2}+184 t^{3}+ \\
599 t^{4}+1648 t^{5}+\cdots\end{array}$ & $\begin{array}{c}1+8 t+34 t^{2}+108 t^{3}+ \\
323 t^{4}+872 t^{5}+\cdots\end{array}$ & $\begin{array}{c}12 t^{2}+76 t^{3}+276 t^{4}+ \\
776 t^{5}+\cdots\end{array}$ \\
\hline$(\mathrm{IV})_{N=2}$ & $\begin{array}{c}1+20 t+224 t^{2}+1843 t^{3}+ \\
12276 t^{4}+69526 t^{5}+\cdots\end{array}$ & $\begin{array}{c}1+20 t+224 t^{2}+1803 t^{3}+ \\
11516 t^{4}+61590 t^{5}+\cdots\end{array}$ & $40 t^{3}+760 t^{4}+7936 t^{5}+\cdots$ \\
\hline
\end{tabular}

Table 20. Coulomb branch Hilbert series for the magnetic quivers of the $Y_{N}^{2,1}$ theory. The corresponding unitary and orthosymplectic quivers are given in table 19.

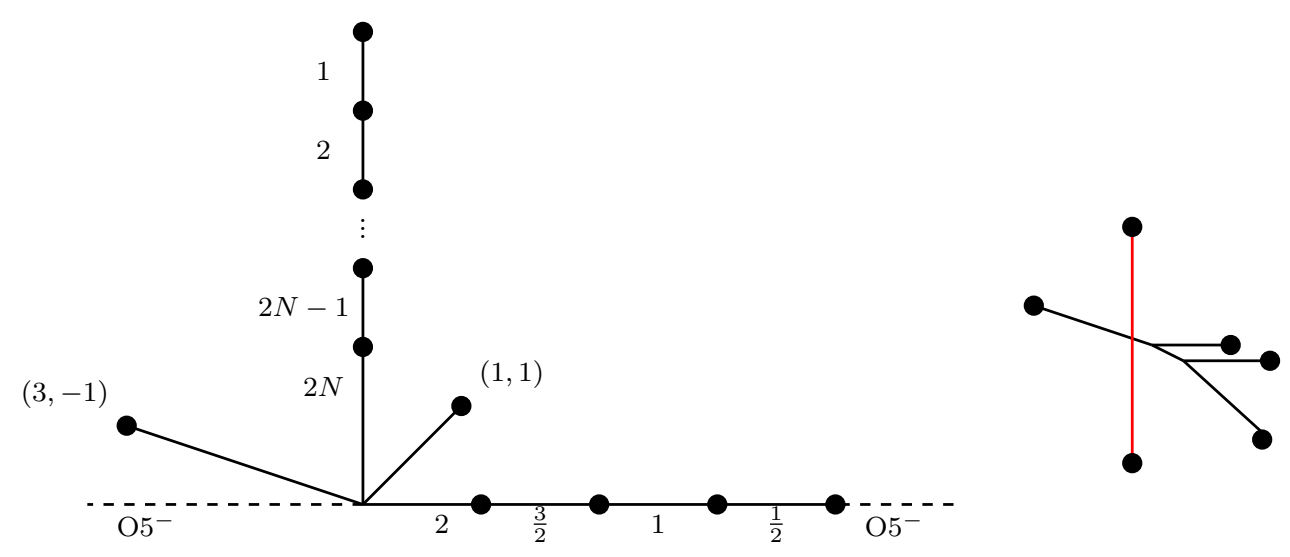

Figure 51. Orientifold web for the $\mathrm{Y}_{N}^{3,1}$ theory along with the maximal subdivision at the centre of the junction.

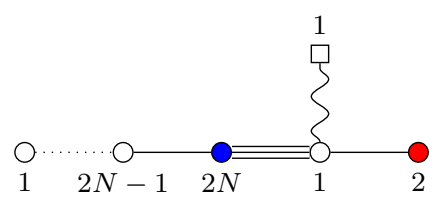

(a) Orthosymplectic quiver

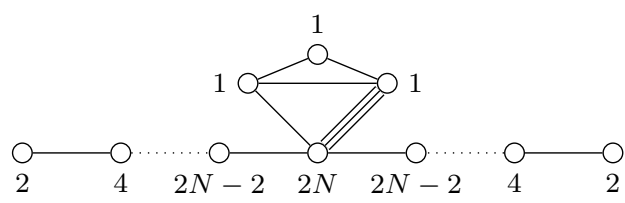

(b) Unitary quiver

Figure 52. The magnetic quivers for the $\mathrm{Y}_{N}^{3,1}$ theory. 


\begin{tabular}{|c|c|c|c|}
\hline \multirow{2}{*}{ Theory } & Unitary & \multicolumn{2}{|c|}{ Orthosymplectic } \\
\cline { 2 - 4 } & $\operatorname{HS}(t)$ & $\operatorname{HS}(t ; \vec{m} \in \mathbb{Z})$ & $\operatorname{HS}\left(t ; \vec{m} \in \mathbb{Z}+\frac{1}{2}\right)$ \\
\hline \multirow{2}{*}{$Y_{1}^{3,1}$} & $1+7 t+38 t^{2}+145 t^{3}+$ & $1+7 t+30 t^{2}+97 t^{3}+$ & $8 t^{2}+48 t^{3}+184 t^{4}+$ \\
& $463 t^{4}+1252 t^{5}+\cdots$ & $279 t^{4}+716 t^{5}+\cdots$ & $536 t^{5}+\cdots$ \\
\hline$Y_{2}^{3,1}$ & $1+19 t+204 t^{2}+1603 t^{3}+$ & $1+19 t+204 t^{2}+1579 t^{3}+$ & $24 t^{3}+432 t^{4}+4336 t^{5}+\cdots$ \\
\hline
\end{tabular}

Table 21. Coulomb branch Hilbert series for the magnetic quivers in the figure 52 for the $Y_{N}^{3,1}$ theory.

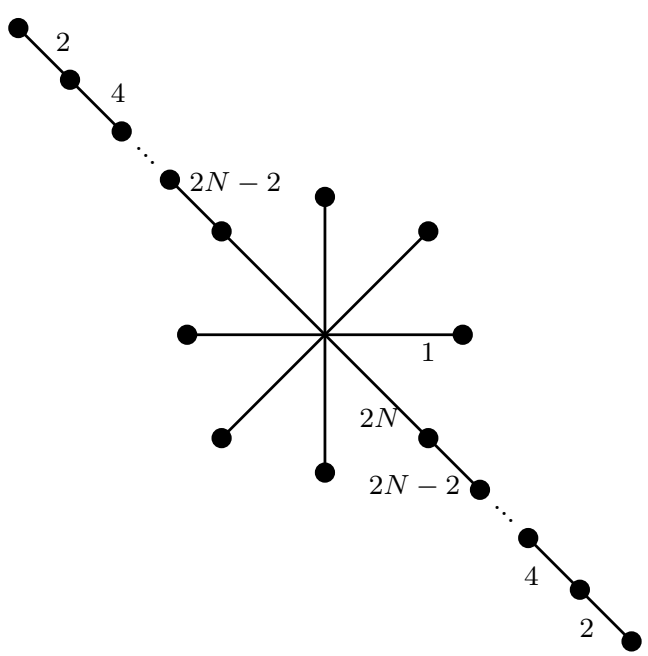



(I)

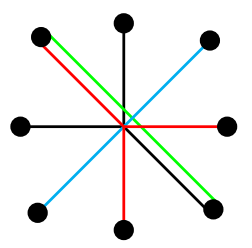

(III)

Figure 53. Ordinary web for the $\mathrm{Y}_{N}^{2,2}$ theory at the fixed point, along with the possible maximal subdivisions of the centre of the junction.

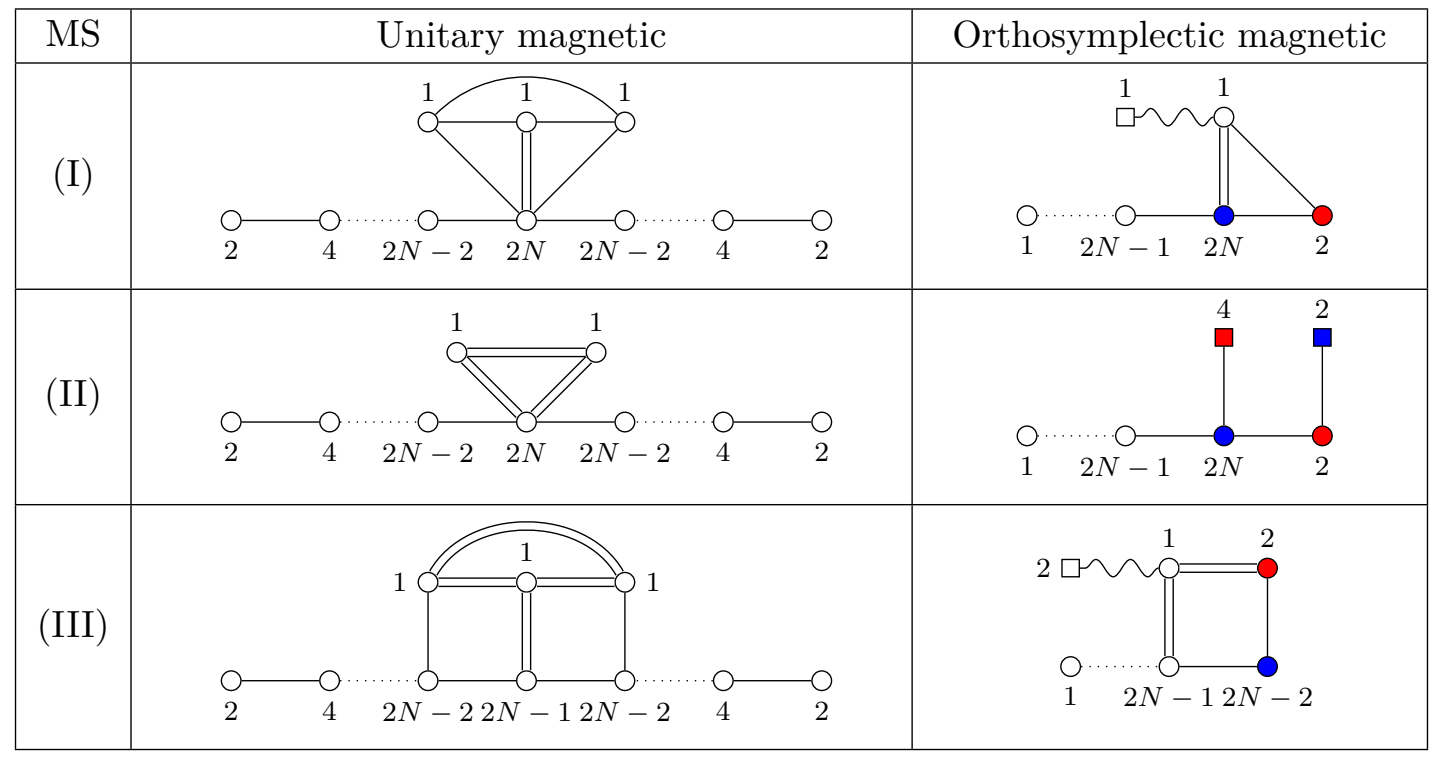

Table 22. The magnetic quivers derived from the various maximal subdivisions (MS) of the unitary and orientifold web diagrams of the $\mathrm{Y}_{N}^{2,2}$ theory. 




Figure 54. Orientifold web for the $\mathrm{Y}_{N}^{2,2}$ theory along with its maximal subdivisions. In the subdivision marked as (II), the blue and red subweb are related to the discrete theta angle from O5 plane and are thus immobile.

\begin{tabular}{|c|c|c|c|}
\hline \multirow{2}{*}{ MS } & Unitary & \multicolumn{2}{|c|}{ Orthosymplectic } \\
\hline & $\operatorname{HS}(t)$ & $\mathrm{HS}(t ; \vec{m} \in \mathbb{Z})$ & $\mathrm{HS}\left(t ; \vec{m} \in \mathbb{Z}+\frac{1}{2}\right)$ \\
\hline$(\mathrm{I})_{N=1}$ & $\begin{array}{c}1+5 t+28 t^{2}+105 t^{3}+ \\
339 t^{4}+920 t^{5}+\cdots\end{array}$ & $\begin{array}{c}1+5 t+20 t^{2}+65 t^{3}+ \\
195 t^{4}+512 t^{5}+\cdots\end{array}$ & $\begin{array}{c}8 t^{2}+40 t^{3}+144 t^{4}+ \\
408 t^{5}+\cdots\end{array}$ \\
\hline$(\mathrm{I})_{N=2}$ & $\begin{array}{c}1+17 t+168 t^{2}+1233 t^{3}+ \\
7427 t^{4}+38575 t^{5}+\cdots\end{array}$ & $\begin{array}{c}1+17 t+168 t^{2}+1209 t^{3}+ \\
7019 t^{4}+34671 t^{5}+\cdots\end{array}$ & $24 t^{3}+408 t^{4}+3904 t^{5}+\cdots$ \\
\hline$(\mathrm{II})_{N=1}$ & $\begin{array}{c}1+4 t+15 t^{2}+45 t^{3}+ \\
110 t^{4}+239 t^{5}+\cdots\end{array}$ & $\begin{array}{c}1+4 t+15 t^{2}+45 t^{3}+ \\
110 t^{4}+239 t^{5}+\cdots\end{array}$ & not required \\
\hline$(\mathrm{II})_{N=2}$ & $\begin{array}{c}1+16 t+151 t^{2}+1041 t^{3}+ \\
5810 t^{4}+27652 t^{5}+\cdots\end{array}$ & $\begin{array}{c}1+16 t+151 t^{2}+1041 t^{3}+ \\
5810 t^{4}+27652 t^{5}+\cdots\end{array}$ & not required \\
\hline$(\mathrm{III})_{N=1}$ & $\begin{array}{c}1+5 t+20 t^{2}+60 t^{3}+ \\
151 t^{4}+331 t^{5}+\cdots\end{array}$ & $\begin{array}{c}1+5 t+16 t^{2}+40 t^{3}+ \\
91 t^{4}+191 t^{5}+\cdots\end{array}$ & $4 t^{2}+20 t^{3}+60 t^{4}+140 t^{5}+\cdots$ \\
\hline$(\mathrm{III})_{N=2}$ & $\begin{array}{c}1+17 t+168 t^{2}+1225 t^{3}+ \\
7255 t^{4}+36626 t^{5}+\cdots\end{array}$ & $\begin{array}{c}1+17 t+168 t^{2}+1209 t^{3}+ \\
6983 t^{4}+34050 t^{5}+\cdots\end{array}$ & $16 t^{3}+272 t^{4}+2576 t^{5}+\cdots$ \\
\hline
\end{tabular}

Table 23. Coulomb branch Hilbert series for magnetic quivers of the $Y_{N}^{2,2}$ theory. The corresponding unitary and orthosymplectic quivers are given in table 22. 


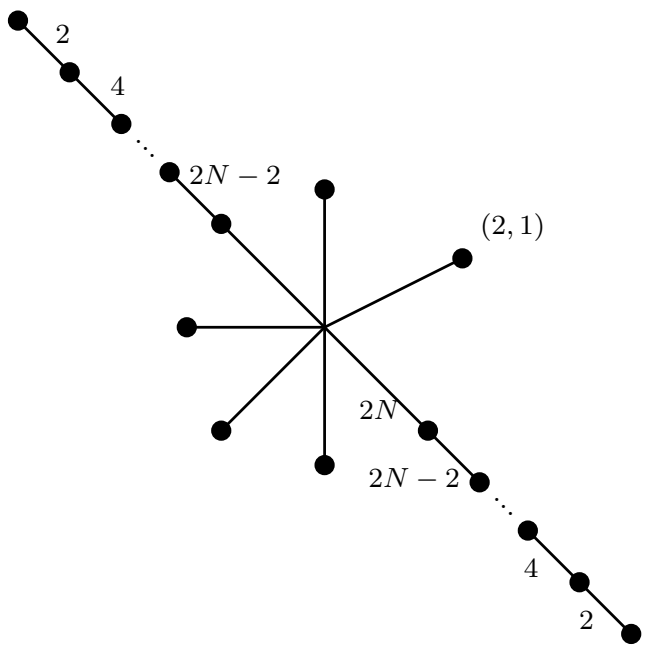

Figure 55. Unitary web description of the $Y_{N}^{2,3}$ theory.

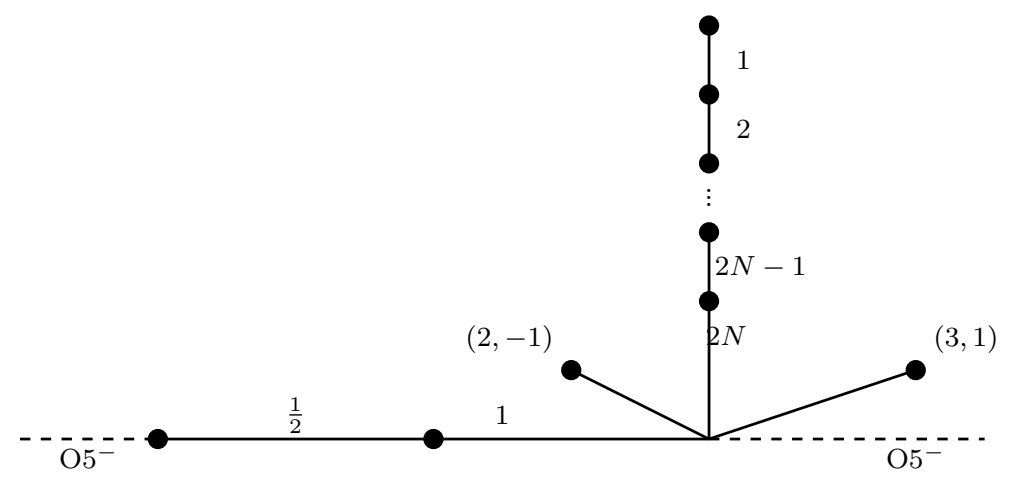

Figure 56. Orientifold web description of the $Y_{N}^{2,3}$ theory.

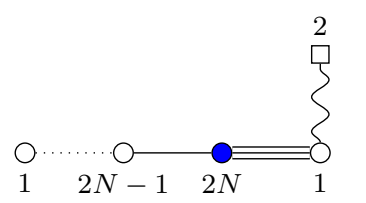

(a) Orthosymplectic quiver



(b) Unitary quiver

Figure 57. The magnetic quivers for the $\mathrm{Y}_{N}^{2,3}$ theory.

\begin{tabular}{|c|c|c|c|}
\hline \multirow{2}{*}{ Theory } & Unitary & \multicolumn{2}{|c|}{ Orthosymplectic } \\
\cline { 2 - 4 } & $\operatorname{HS}(t)$ & $\operatorname{HS}(t ; \vec{m} \in \mathbb{Z})$ & $\operatorname{HS}\left(t ; \vec{m} \in \mathbb{Z}+\frac{1}{2}\right)$ \\
\hline$Y_{1}^{2,3}$ & $\begin{array}{c}1+4 t+17 t^{2}+47 t^{3}+ \\
120 t^{4}+255 t^{5}+\cdots\end{array}$ & $\begin{array}{c}1+4 t+13 t^{2}+31 t^{3}+ \\
72 t^{4}+147 t^{5}+\cdots\end{array}$ & $4 t^{2}+16 t^{3}+48 t^{4}+108 t^{5}+\cdots$ \\
\hline$Y_{2}^{2,3}$ & $\begin{array}{c}1+16 t+151 t^{2}+1051 t^{3}+ \\
5940 t^{4}+28640 t^{5}+\cdots\end{array}$ & $\begin{array}{c}1+16 t+151 t^{2}+1039 t^{3}+ \\
5748 t^{4}+26892 t^{5}+\cdots\end{array}$ & $12 t^{3}+192 t^{4}+1748 t^{5}+\cdots$ \\
\hline
\end{tabular}

Table 24. Coulomb branch Hilbert series for the magnetic quivers in the figure 57 for the $Y_{N}^{2,3}$ theory. 


\section{List of Figures}

1 5-brane web for the $\#_{M, N}$ theory. 5

2 Obtaining web diagrams without O5-planes via gauging. 5

3 Unitary web diagram of $\#_{3, N}$ theory. 6

$4 \quad$ Orientifold web diagram of $\mathrm{K}_{N}^{1}$ theory. $\quad 10$

$5 \quad$ Unitary web diagram of $\mathrm{K}_{N}^{1}$ theory. 10

$6 \quad$ Orientifold web diagram of $\mathrm{K}_{N}^{2}$ theory. 10

7 Unitary web diagram of $\mathrm{K}_{N}^{2}$ theory. 11

$8 \quad$ Orientifold web diagram of $\mathrm{K}_{N}^{3}$ theory. 11

9 Unitary web diagram of $\mathrm{K}_{N}^{3}$ theory. 12

10 Orientifold web diagram of $\mathrm{Y}_{N}^{1,1}$ theory. 16

11 Unitary web diagram of $\mathrm{Y}_{N}^{1,1}$ theory. 16

12 Origin of the exotic hypermultiplets. 17

13 Constructing web diagrams for $\mathrm{Y}_{N}^{2 \times 1,2 \times 1}$ theory by gauging $\mathrm{SU}(2)$ 's. 21

14 Origin of the antisymmetric matter. 21

15 Usual 5-brane web diagrams for $E_{1}$ and $\tilde{E}_{1}$ SCFT. 21

16 5-brane web diagrams with O5-plane for $E_{1}$ and $\tilde{E}_{1}$ SCFTs. 22

17 5-brane web diagram with O5-plane for $E_{3}$ SCFT. 22

18 Decomposable and not decomposable orientifold web diagrams in $Y_{N}^{2 \times 1,2 \times 1} .23$

19 Orientifold web diagram for $Y_{N}^{2 \times 1,2 \times 1}$ theory. 24

20 Unitary web diagram for $Y_{N}^{2 \times 1,2 \times 1}$ theory. 25

21 Orientifold web diagram for $H_{N}^{N, N}$ theory. 29

22 Unitary web diagram for $H_{N}^{N, N}$ theory. 29

23 Orientifold web diagram for $+_{1, N}$ theory. 31

24 Unitary web diagram for $+_{1, N}$ theory. 31

25 Magnetic quivers for $+_{1, N}$ theory. 32

26 Orientifold web diagram for $\hat{K}_{N}^{1}$ theory. 32

27 Unitary web diagram for $\hat{K}_{N}^{1}$ theory. 33

28 Magnetic quivers for $\hat{K}_{N}^{1}$ theory. 34

29 Orientifold web diagram for $X_{N}^{1,1}$ theory. 35

30 Unitary web diagram for $X_{N}^{1,1}$ theory. 35

31 Orientifold web diagram for the $+{ }_{N}^{3,1}$ theory. 37

32 Unitary web diagram for $+_{N}^{3,1}$ theory. 37

33 S-dual of $\#_{3,2}$ theory. 38

34 5-brane web for $+{ }_{N}^{3,1}$ theory obtained from $\#_{3,2}$ theory. 38

35 Usual 5-brane web for $A_{3}$ quiver for $N=2 \quad 38$

$36 \quad 5$-brane web obtained from the $A_{3}$ quiver by the decoupling. 38

37 Magnetic quivers for the $+_{N}^{3,1}$ theory. 40

38 An orientifold web for the $\tilde{K}_{N}^{1}$ theory. 41

39 A unitary web description of the $\tilde{K}_{N}^{1}$ theory. 41

40 Orientifold and unitary web diagrams for $+_{N}$ theory. 44

41 Magnetic quivers for the $+_{N}$ theory 44 
42 Orientifold and unitary web diagram for the $\kappa_{N}^{1}$ theory. 45

43 Magnetic quivers for the $\kappa_{N}^{1}$ theory 46

44 Web diagram with external $(2 k-1,2)$ and $(2 k-1,-2) 5$-branes. 55

45 Orientifold web diagram for theory in figure 44.

46 Web diagram with external $(6 k-1,3)$ and $(6 k-1,-3) 5$-branes. 55

47 Orientifold web diagram for the theory in figure 46.

48 Ordinary web diagram for $\mathrm{Y}_{N}^{2,1}$ theory. 57

49 Orientifold web diagram for $\mathrm{Y}_{N}^{2,1}$ theory. 58

50 Unitary web diagram for $\mathrm{Y}_{N}^{3,1}$ theory. $\quad 58$

51 Orientifold web diagram for $\mathrm{Y}_{N}^{3,1}$ theory. $\quad 60$

52 The magnetic quivers for the $\mathrm{Y}_{N}^{3,1}$ theory. 60

53 Unitary web diagram for $\mathrm{Y}_{N}^{2,2}$ theory. 61

54 Orientifold web diagram for $\mathrm{Y}_{N}^{2,2}$ theory. 62

55 Unitary web diagram for $\mathrm{Y}_{N}^{2,3}$ theory. 63

56 Orientifold web diagram for $\mathrm{Y}_{N}^{2,3}$ theory. 63

57 The magnetic quivers for the $\mathrm{Y}_{N}^{2,3}$ theory. 63

\section{List of Tables}

1 Coulomb branch HS for magnetic quivers of $\#_{3, N}$ theory. 7

$2 \quad$ Magnetic quivers for $\mathrm{K}_{N}^{p}$ family. 13

3 Coulomb branch HS for magnetic quivers of $K_{N}^{p}$ family. 14

$4 \quad$ Magnetic quivers for $\mathrm{Y}_{N}^{1,1}$ theory. 18

$5 \quad$ Coulomb branch HS for magnetic quivers of $Y_{N}^{1,1}$ theory. 19

$6 \quad$ Magnetic quivers for $Y_{N}^{2 \times 1,2 \times 1}$ theory. 26

$7 \quad$ Coulomb branch HS for magnetic quivers of $Y_{N}^{2 \times 1,2 \times 1}$ theory. 27

$8 \quad$ Magnetic quivers for $H_{N}^{N, N}$ family. 29

9 Coulomb branch HS for magnetic quivers of $H_{N}^{N, N}$ family. 29

10 Coulomb branch HS for magnetic quivers of $+{ }_{1, N}$ theory. 32

11 Higgs branch HS for magnetic quivers of $\hat{K}_{N}^{1}$ theory. 34

12 Magnetic quivers for $X_{N}^{1,1}$ theory. 36

13 Coulomb branch HS for magnetic quivers of $X_{N}^{1,1}$ theory. 36

14 Magnetic quivers for $\tilde{K}_{N}^{1}$ theory. 41

15 Coulomb branch HS for magnetic quivers of $\tilde{K}_{N}^{1}$ theory. 42

16 Coulomb branch HS for magnetic quivers of $+_{N}$ theory. 44

17 Coulomb branch HS for magnetic quivers of $\kappa_{N}^{1}$ theory. 46

18 Palindromic polynomials appearing in the main sections. 53

$19 \begin{array}{ll}\text { Magnetic quivers for } \mathrm{Y}_{N}^{2,1} \text { theory. } & 59\end{array}$

20 Coulomb branch HS for $\mathrm{Y}_{N}^{2,1}$ theory. 60

21 Coulomb branch HS for magnetic quivers of $\mathrm{Y}_{N}^{3,1}$ theory. 61

22 Magnetic quivers for $\mathrm{Y}_{N}^{2,2}$ theory. $\quad 61$

23 Coulomb branch HS for magnetic quivers of $\mathrm{Y}_{N}^{2,2}$ theory. 62

24 Coulomb branch HS for magnetic quivers of $\mathrm{Y}_{N}^{2,3}$ theory. 63 
Open Access. This article is distributed under the terms of the Creative Commons Attribution License (CC-BY 4.0), which permits any use, distribution and reproduction in any medium, provided the original author(s) and source are credited.

\section{References}

[1] A. Bourget, J.F. Grimminger, A. Hanany, M. Sperling and Z. Zhong, Magnetic Quivers from Brane Webs with O5 Planes, JHEP 07 (2020) 204 [arXiv:2004.04082] [INSPIRE].

[2] N. Seiberg, Five-dimensional SUSY field theories, nontrivial fixed points and string dynamics, Phys. Lett. B 388 (1996) 753 [hep-th/9608111] [INSPIRE].

[3] K.A. Intriligator, D.R. Morrison and N. Seiberg, Five-dimensional supersymmetric gauge theories and degenerations of Calabi-Yau spaces, Nucl. Phys. B 497 (1997) 56 [hep-th/9702198] [INSPIRE].

[4] D.R. Morrison and N. Seiberg, Extremal transitions and five-dimensional supersymmetric field theories, Nucl. Phys. B 483 (1997) 229 [hep-th/9609070] [INSPIRE].

[5] H.-C. Kim, S.-S. Kim and K. Lee, 5-dim Superconformal Index with Enhanced En Global Symmetry, JHEP 10 (2012) 142 [arXiv:1206.6781] [INSPIRE].

[6] D. Bashkirov, A comment on the enhancement of global symmetries in superconformal $\mathrm{SU}(2)$ gauge theories in 5D, arXiv:1211.4886 [INSPIRE].

[7] A. Iqbal and C. Vafa, BPS Degeneracies and Superconformal Index in Diverse Dimensions, Phys. Rev. D 90 (2014) 105031 [arXiv:1210.3605] [InSPIRE].

[8] D. Rodríguez-Gómez and G. Zafrir, On the $5 d$ instanton index as a Hilbert series, Nucl. Phys. B 878 (2014) 1 [arXiv:1305.5684] [inSPIRE].

[9] M. Taki, Notes on Enhancement of Flavor Symmetry and 5d Superconformal Index, arXiv:1310.7509 [INSPIRE].

[10] O. Bergman, D. Rodríguez-Gómez and G. Zafrir, Discrete $\theta$ and the $5 d$ superconformal index, JHEP 01 (2014) 079 [arXiv: 1310.2150] [INSPIRE].

[11] O. Bergman, D. Rodríguez-Gómez and G. Zafrir, 5-Brane Webs, Symmetry Enhancement, and Duality in 5d Supersymmetric Gauge Theory, JHEP 03 (2014) 112 [arXiv:1311.4199] [INSPIRE].

[12] O. Bergman and G. Zafrir, Lifting 4d dualities to 5d, JHEP 04 (2015) 141 [arXiv: 1410.2806] [INSPIRE].

[13] C. Hwang, J. Kim, S. Kim and J. Park, General instanton counting and 5d SCFT, JHEP 07 (2015) 063 [Addendum ibid. 04 (2016) 094] [arXiv: 1406.6793] [INSPIRE].

[14] G. Zafrir, Duality and enhancement of symmetry in 5d gauge theories, JHEP 12 (2014) 116 [arXiv: 1408.4040] [INSPIRE].

[15] L. Bao, V. Mitev, E. Pomoni, M. Taki and F. Yagi, Non-Lagrangian Theories from Brane Junctions, JHEP 01 (2014) 175 [arXiv:1310.3841] [INSPIRE].

[16] S.-S. Kim, M. Taki and F. Yagi, Tao Probing the End of the World, PTEP 2015 (2015) 083B02 [arXiv: 1504.03672] [INSPIRE].

[17] H. Hayashi, H.-C. Kim and T. Nishinaka, Topological strings and $5 d T_{N}$ partition functions, JHEP 06 (2014) 014 [arXiv:1310.3854] [inSPIRE]. 
[18] V. Mitev, E. Pomoni, M. Taki and F. Yagi, Fiber-Base Duality and Global Symmetry Enhancement, JHEP 04 (2015) 052 [arXiv: 1411.2450] [INSPIRE].

[19] S.-S. Kim and F. Yagi, 5d En Seiberg-Witten curve via toric-like diagram, JHEP 06 (2015) 082 [arXiv: 1411.7903] [INSPIRE].

[20] H. Hayashi and G. Zoccarato, Topological vertex for Higgsed $5 d T_{N}$ theories, JHEP 09 (2015) 023 [arXiv: 1505.00260] [INSPIRE].

[21] H. Hayashi, S.-S. Kim, K. Lee, M. Taki and F. Yagi, A new 5d description of $6 d$ D-type minimal conformal matter, JHEP 08 (2015) 097 [arXiv: 1505.04439] [INSPIRE].

[22] H. Hayashi, S.-S. Kim, K. Lee and F. Yagi, Discrete theta angle from an O5-plane, JHEP 11 (2017) 041 [arXiv: 1707.07181] [INSPIRE]

[23] H. Hayashi, S.-S. Kim, K. Lee and F. Yagi, Dualities and 5-brane webs for 5d rank 2 SCFTs, JHEP 12 (2018) 016 [arXiv:1806.10569] [INSPIRE].

[24] H. Hayashi, S.-S. Kim, K. Lee and F. Yagi, Complete prepotential for $5 d \mathcal{N}=1$ superconformal field theories, JHEP 02 (2020) 074 [arXiv:1912.10301] [INSPIRE].

[25] N. Lambert, C. Papageorgakis and M. Schmidt-Sommerfeld, Instanton Operators in Five-Dimensional Gauge Theories, JHEP 03 (2015) 019 [arXiv:1412.2789] [INSPIRE].

[26] D. Rodriguez-Gomez and J. Schmude, Supersymmetrizing 5d instanton operators, JHEP 03 (2015) 114 [arXiv: 1501.00927] [inSPIRE].

[27] Y. Tachikawa, Instanton operators and symmetry enhancement in 5d supersymmetric gauge theories, PTEP 2015 (2015) 043B06 [arXiv:1501.01031] [INSPIRE].

[28] G. Zafrir, Instanton operators and symmetry enhancement in 5d supersymmetric USp, SO and exceptional gauge theories, JHEP 07 (2015) 087 [arXiv: 1503.08136] [INSPIRE].

[29] K. Yonekura, Instanton operators and symmetry enhancement in 5d supersymmetric quiver gauge theories, JHEP 07 (2015) 167 [arXiv:1505.04743] [INSPIRE].

[30] O. Aharony and A. Hanany, Branes, superpotentials and superconformal fixed points, Nucl. Phys. B 504 (1997) 239 [hep-th/9704170] [INSPIRE].

[31] O. Aharony, A. Hanany and B. Kol, Webs of $(p, q)$ five-branes, five-dimensional field theories and grid diagrams, JHEP 01 (1998) 002 [hep-th/9710116] [INSPIRE].

[32] O. DeWolfe, A. Hanany, A. Iqbal and E. Katz, Five-branes, seven-branes and five-dimensional $E_{n}$ field theories, JHEP 03 (1999) 006 [hep-th/9902179] [INSPIRE].

[33] M.R. Gaberdiel and B. Zwiebach, Exceptional groups from open strings, Nucl. Phys. B 518 (1998) 151 [hep-th/9709013] [INSPIRE].

[34] M.R. Gaberdiel, T. Hauer and B. Zwiebach, Open string-string junction transitions, Nucl. Phys. B 525 (1998) 117 [hep-th/9801205] [INSPIRE].

[35] D. Gaiotto and H.-C. Kim, Duality walls and defects in $5 d \mathcal{N}=1$ theories, JHEP 01 (2017) 019 [arXiv: 1506.03871] [INSPIRE].

[36] G. Zafrir, Brane webs, 5d gauge theories and $6 d \mathcal{N}=(1,0)$ SCFT's, JHEP 12 (2015) 157 [arXiv: 1509.02016] [INSPIRE].

[37] H. Hayashi, S.-S. Kim, K. Lee and F. Yagi, 6d SCFTs, 5d Dualities and Tao Web Diagrams, JHEP 05 (2019) 203 [arXiv:1509.03300] [INSPIRE]. 
[38] H. Hayashi, S.-S. Kim, K. Lee, M. Taki and F. Yagi, More on 5d descriptions of 6d SCFTs, JHEP 10 (2016) 126 [arXiv:1512.08239] [INSPIRE].

[39] P. Jefferson, S. Katz, H.-C. Kim and C. Vafa, On Geometric Classification of 5d SCFTs, JHEP 04 (2018) 103 [arXiv:1801.04036] [INSPIRE].

[40] S. Cremonesi, G. Ferlito, A. Hanany and N. Mekareeya, Instanton Operators and the Higgs Branch at Infinite Coupling, JHEP 04 (2017) 042 [arXiv: 1505.06302] [INSPIRE].

[41] G. Ferlito, A. Hanany, N. Mekareeya and G. Zafrir, 3d Coulomb branch and 5d Higgs branch at infinite coupling, JHEP 07 (2018) 061 [arXiv:1712.06604] [INSPIRE].

[42] F. Benini, S. Benvenuti and Y. Tachikawa, Webs of five-branes and $N=2$ superconformal field theories, JHEP 09 (2009) 052 [arXiv:0906.0359] [INSPIRE].

[43] F. Benini, Y. Tachikawa and D. Xie, Mirrors of 3d Sicilian theories, JHEP 09 (2010) 063 [arXiv: 1007.0992] [INSPIRE].

[44] J. Eckhard, S. Schäfer-Nameki and Y.-N. Wang, Trifectas for $T_{N}$ in 5d, JHEP 07 (2020) 199 [arXiv: 2004.15007] [INSPIRE].

[45] S. Cabrera, A. Hanany and F. Yagi, Tropical Geometry and Five Dimensional Higgs Branches at Infinite Coupling, JHEP 01 (2019) 068 [arXiv:1810.01379] [INSPIRE].

[46] S. Cabrera, A. Hanany and M. Sperling, Magnetic quivers, Higgs branches, and $6 d N=(1,0)$ theories, JHEP 06 (2019) 071 [Erratum ibid. 07 (2019) 137] [arXiv: 1904.12293] [INSPIRE].

[47] O. Bergman, D. Rodríguez-Gómez and C.F. Uhlemann, Testing $A d S_{6} / C F T_{5}$ in Type IIB with stringy operators, JHEP 08 (2018) 127 [arXiv: 1806.07898] [INSPIRE].

[48] G. Zafrir, Brane webs in the presence of an $\mathrm{O5}^{-}$-plane and $4 d$ class $S$ theories of type D, JHEP 07 (2016) 035 [arXiv:1602.00130] [INSPIRE].

[49] D. Gaiotto and E. Witten, S-duality of Boundary Conditions In $N=4$ Super Yang-Mills Theory, Adv. Theor. Math. Phys. 13 (2009) 721 [arXiv:0807.3720] [InSPIRE].

[50] Z. Zhong, Quiver gauge theories in 3d, 5d and 6d, Master's thesis, Imperial College, London, U.K., (2018).

[51] A. Bourget, J.F. Grimminger, A. Hanany, R. Kalveks, M. Sperling and Z. Zhong, Magnetic Lattices for Orthosymplectic Quivers, arXiv:2007.04667 [INSPIRE].

[52] M. van Beest, A. Bourget, J. Eckhard and S. Schäfer-Nameki, (Symplectic) Leaves and ( $5 d$ Higgs) Branches in the Poly(go)nesian Tropical Rain Forest, JHEP 11 (2020) 124 [arXiv: 2008.05577] [INSPIRE].

[53] O. Bergman and G. Zafrir, 5d fixed points from brane webs and O7-planes, JHEP 12 (2015) 163 [arXiv: 1507.03860] [INSPIRE].

[54] M. Akhond, F. Carta, S. Dwivedi, H. Hayashi, S.-S. Kim and F. Yagi, work in progress.

[55] A. Bourget, J.F. Grimminger, A. Hanany, M. Sperling, G. Zafrir and Z. Zhong, Magnetic quivers for rank 1 theories, JHEP 09 (2020) 189 [arXiv:2006.16994] [INSPIRE].

[56] C. Closset, S. Schäfer-Nameki and Y.-N. Wang, Coulomb and Higgs Branches from Canonical Singularities: Part 0, arXiv:2007.15600 [INSPIRE].

[57] S. Cremonesi, A. Hanany and A. Zaffaroni, Monopole operators and Hilbert series of Coulomb branches of $3 d \mathcal{N}=4$ gauge theories, JHEP 01 (2014) 005 [arXiv:1309.2657] [INSPIRE]. 